\title{
Measured quantum groupoids associated to proper dynamical quantum groups
}

\author{
Thomas Timmermann*
}

\begin{abstract}
Dynamical quantum groups were introduced by Etingof and Varchenko in connection with the dynamical quantum Yang-Baxter equation, and measured quantum groupoids were introduced by Enock, Lesieur and Vallin in their study of inclusions of type $\mathrm{II}_{1}$ factors. In this article, we associate to suitable dynamical quantum groups, which are purely algebraic objects, Hopf $C^{*}$-bimodules and measured quantum groupoids on the level of von Neumann algebras. Assuming invariant integrals on the dynamical quantum group, we first construct a fundamental unitary which yields Hopf bimodules on the level of $C^{*}$-algebras and von Neumann algebras. Next, we assume properness of the dynamical quantum group and lift the integrals to the operator algebras. In a subsequent article, this construction shall be applied to the dynamical $\mathrm{SU}_{q}(2)$ studied by Koelink and Rosengren.
\end{abstract}

Mathematics Subject Classification (2010). 46L99; 81R50, 20G42, 16 T25.

Keywords. Quantum groupoid, dynamical quantum group, Hopf algebroid.

\section{Introduction}

Dynamical quantum groups were introduced by Etingof and Varchenko as an algebraic framework for the study of the dynamical quantum Yang-Baxter equation $[6,7,8]$, a variant of the Yang-Baxter equation arising in statistical mechanics. Every (rigid) solution of this equation has a naturally associated tensor category of representations which turns out to be equivalent to the category of representations of some dynamical quantum group. In the case of the basic rational or basic trigonometric solution, this dynamical quantum group can be regarded as a quantization of the function algebra on some Poisson-Lie-groupoid. In general, it can be regarded as a quantum groupoid and fits into the theory of Hopf algebroids developed by Böhm and others [1].

Measured quantum groupoids were introduced by Enock, Lesieur and Vallin [2, 13] to capture generalized Galois symmetries of certain inclusions of type $\mathrm{II}_{1}$ factors $[3,4,15]$. Apart from this fundamental example in von Neumann algebra theory,

\footnotetext{
*Supported by the SFB 878 "Groups, geometry and actions" funded by the DFG.
} 
which was also considered in the algebraic setting [9, 19], and from the finite case, only few measured quantum groupoids have been constructed and investigated yet $[13,29]$.

Up to now, connections between algebraic and operator-algebraic approaches to quantum groupoids have only been explored in the finite case $[14,17,28]$ and in the form of a few examples and constructions that exist on both levels. The situation is very different in the area of quantum groups, where Woronowicz's theory of compact quantum groups [35] and van Daele's theory of multiplier Hopf algebras with integrals $[12,31]$ form a bridge between the algebraic and operator-algebraic approaches, combining the computational convenience of the former with the power and richness of the latter.

Another approach to quantum groupoids which is equivalent to the algebraic and operator algebraic one, at least in finite dimensions, is via fusion categories [5, 19].

In this article, we associate to suitable dynamical quantum groups, which are purely algebraic objects, Hopf $C^{*}$-bimodules and measured quantum groupoids on the level of von Neumann algebras. The main example of a dynamical group we have in mind for application is the dynamical $\mathrm{SU}_{q}(2)$ studied by Koelink and Rosengren [10], and in a subsequent article, we want to study the construction for this example in detail.

On the dynamical quantum groups, we have to impose several assumptions.

First, we need a left- and a right-invariant integral, which correspond to fiber-wise integration on a groupoid, and a weight on the basis that is suitably quasi-invariant, such that the resulting total integrals are faithful, positive, and coincide. In the case of the dynamical $\mathrm{SU}_{q}(2)$, the left- and right-invariant integrals can be obtained from a Peter-Weyl decomposition due to Koelink and Rosengren [10], while the quasiinvariant weight on the basis can be chosen quite freely.

Second, we assume the dynamical quantum group to be proper, which is the natural analogue of compactness and unitality for quantum groupoids, and to possess a specific approximate unit in the base algebra. The dynamical $\mathrm{SU}_{q}(2)$ mentioned above even is compact and thus satisfies this second assumption.

In particular, the dynamical quantum group need not be a Hopf algebroid, but only a multiplier Hopf algebroid in the sense of [25]. The latter are closely related to the weak multiplier Hopf algebras that were recently introduced by Van Daele and Wang [33, 34].

Third, we assume that the quasi-invariant weight on the basis admits a bounded GNS-construction. Like the first condition, this one is very natural. In the case of the dynamical $\mathrm{SU}_{q}(2)$, the base algebra is formed by all meromorphic functions on the plane and does not admit any non-trivial bounded representations. To apply our construction, one therefore has to change the base and check that the Peter-Weyl decomposition persists.

Given these assumptions, the measured quantum groupoid is constructed as follows. 
The algebraic GNS-construction, applied to the total integral on the dynamical quantum group, yields a Hilbert space of square-integrable functions on the dynamical quantum group together with a natural representation by densely defined multiplication operators. To obtain a $C^{*}$-algebra or von Neumann algebra, one has to show that these multiplication operators are bounded. To prove this and to lift the comultiplication to the resulting $C^{*}$-algebra and von Neumann algebra, we proceed as in the case of quantum groups [23] and construct a fundamental unitary which is pseudo-multiplicative on the level of von Neumann algebras and $C^{*}$-algebras in the sense of [27] and [24], respectively. The general theory of these unitaries then yields completions of the dynamical quantum group in the form a Hopf $C^{*}$-bimodule and a Hopf von-Neumann bimodule, and simultaneously a Pontrjagin dual in the same form. Finally, we extend the invariant integrals to the level of operator algebras, using properness of the dynamical quantum group and standard von Neumann algebra techniques.

This article is organized as follows.

Section 2 provides the algebraic basics on dynamical quantum groups and integration that are needed for the construction in Section 3. We first generalize the definition of a dynamical quantum group or $\mathfrak{h}$-Hopf algebroid, allowing the base to be non-unital, then consider left- and right-invariant integrals on the total algebra and quasi-invariant weights on the basis, and finally construct a $*$-algebra related to the Pontrjagin dual. The main result of this section is the existence of a modular automorphism for the total integral, which follows from a strong invariance property similarly as in the setting of multiplier Hopf algebras [31].

Section 3 presents the construction of the measured quantum groupoid outlined above. It uses Connes spatial theory, in particular the relative tensor product of Hilbert modules, and the $C^{*}$-algebraic analogue of that construction [22], and introduces the necessary concepts along the way when they are needed.

We use standard notation and adopt the following conventions. All algebras will be over the ground field $\mathbb{C}$ and we do not assume the existence of a unit element. Given a vector space $V$ with a subset $X \subseteq V$, we denote by $\langle X\rangle \subseteq V$ the linear span and, if $V$ is normed, by $[X] \subseteq V$ the closed linear span of $X$. Inner products on Hilbert spaces will be linear in the second and anti-linear in the first variable.

\section{Dynamical quantum groups with integrals on the algebraic level}

This section summarizes and develops the basics on dynamical quantum groups and integration used in this article. Before turning to details, let us outline the main concepts.

A dynamical quantum group is a special quantum groupoid and as such consists of an algebra $B$ called the basis, an algebra $A$, an embedding $r: B \rightarrow A$ and an anti-homomorphic embedding $s: B \rightarrow A$ whose images commute, and a 
comultiplication, antipode and counit. What makes it special is that the basis $B$ is commutative, that $r(B)$ and $s(B)$ are central in $A$ up to a twist which is controlled by an action of a group $\Gamma$ on $B$ and a bigrading of $A$ by $\Gamma$, and that the target of the comultiplication is a well-behaved monoidal product $A \tilde{\otimes} A$.

Integration on a quantum groupoid involves several ingredients. The analogue of the left- or right-invariance property of Haar measures on groups, Haar systems on groupoids, and Haar weights on quantum groups can be formulated for conditional expectations from $A$ to $r(B)$ or $s(B)$, respectively. To obtain a total integration on $A$, such a partial integral has to be composed with a suitable functional on $B$ that is quasi-invariant with respect to the action of $\Gamma$.

Let us now turn to details. We proceed as follows.

From the beginning, we assume all our algebras to possess an involution but not necessarily a unit. We first recall terminology concerning non-unital algebras (\$2.1), then describe the monoidal product $A \tilde{\otimes} A(\S 2.2)$, and define dynamical quantum groups or, more precisely, multiplier $(B, \Gamma)$-Hopf $*$-algebroids ( $(2.3)$. Afterwards, we introduce and study integrals $(\$ 2.4-\$ 2.6)$ and prove the existence of a modular automorphism that controls the deviation of the total integral from being a trace. Using integration, we finally construct the dual $*$-algebra of a multiplier $(B, \Gamma)$ Hopf $*$-algebroid $(\$ 2.7)$.

2.1. Preliminaries on non-unital algebras. To handle non-unital algebras, we use extra non-degeneracy assumptions and multiplier algebras [30, appendix] which are recalled below.

Let $R$ be an algebra, not necessarily unital. Given a left $R$-module $M$, we say that $R$ has local units for $M$ if for each finite subset $F \subseteq M$, there exists some $r \in R$ such that $r m=m$ for all $m \in F$ [32]. The corresponding notion for right $R$-modules is defined similarly. We say that $R$ has local units if it has local units for $R$, regarded as a left and as a right $R$-module.

Let $R$ and $S$ be algebras with local units, let $N$ be an $R$-S -bimodule and assume that $R$ and $S$ have local units for $N$. A multiplier of $N$ is a pair $T=\left(T_{\rho}, T_{\lambda}\right)$, where $T_{\rho}: R \rightarrow N$ is a left $R$-module map and $T_{\lambda}: S \rightarrow N$ a right $S$-module map satisfying $T_{\rho}(r) s=r T_{\lambda}(s)$ for all $r \in R, s \in S$. Given such a multiplier, we write $r T:=T_{\rho}(r)$ and $T s:=T_{\lambda}(s)$ for all $r \in R, s \in S$. We denote the set of all multipliers of $N$ by $M(N)$. Clearly, $N$ embeds into $M(N)$ and $M(N)$ carries a natural structure of an $R-S$-bimodule that is compatible with this embedding.

Regarding $R$ as an $R$ - $R$-bimodule, $M(R)$ becomes an algebra via $T T^{\prime}=\left(T_{\rho}^{\prime} \circ T_{\rho}\right.$, $\left.T_{\lambda} \circ T_{\lambda}^{\prime}\right)$, and $R$ embeds into $M(R)$ as an essential ideal. If $R$ is a $*$-algebra, then so is $M(R)$, where the adjoint of a multiplier $T=\left(T_{\rho}, T_{\lambda}\right) \in M(R)$ is the pair $T^{*}=\left(T_{\rho}^{*}, T_{\lambda}^{*}\right)$ given by $T_{\rho}^{*}(r)=\left(T_{\lambda}\left(r^{*}\right)\right)^{*}$ and $T_{\lambda}^{*}(r)=\left(T_{\rho}\left(r^{*}\right)\right)^{*}$ for all $r \in R$.

The bimodule $N$ is an $M(R)-M(S)$-bimodule via $T(r n s) T^{\prime}:=T_{\lambda}(r) n T_{\rho}^{\prime}(s)$ for all $T \in M(R), r \in R, n \in N, s \in S, T^{\prime} \in M(S)$, and $M(N)$ is an $M(R)$ $M(S)$-bimodule via $T T^{\prime} T^{\prime \prime}:=\left(T_{\rho}^{\prime \prime} \circ T_{\rho}^{\prime} \circ T_{\rho}, T_{\lambda} \circ T_{\lambda}^{\prime} \circ T_{\lambda}^{\prime \prime}\right)$ for all $T \in M(R)$, $T^{\prime} \in M(N), T^{\prime \prime} \in M(S)$. 
A homomorphism $\pi: R \rightarrow M(S)$ is non-degenerate if $\langle\pi(R) S\rangle=S=$ $\langle S \pi(R)\rangle$; in that case, it extends uniquely to a homomorphism $M(R) \rightarrow M(S)$ which is again denoted by $\pi$ (see [30]).

2.2. The category of $(B, \Gamma)^{\mathrm{ev}}$-algebras. Let $B$ be a commutative $*$-algebra with local units, let $\Gamma$ be a group that acts on $B$ on the left, and let $e \in \Gamma$ be the unit.

A $(B, \Gamma)$-module is a $\Gamma$-graded $B$-bimodule $V=\bigoplus_{\gamma \in \Gamma} V_{\gamma}$ for which $B$ has local units, where each $V_{\gamma}$ is a $B$-bimodule and $v b=\gamma(b) v$ for all $v \in V_{\gamma}$, $b \in B, \gamma \in \Gamma$. A morphism of $(B, \Gamma)$-modules $V$ and $W$ is a morphism of $\Gamma$-graded $B$-bimodules.

A $(B, \Gamma)$-algebra is a $\Gamma$-graded $*$-algebra $A=\bigoplus_{\gamma \in \Gamma} A_{\gamma}$ which has local units in $A_{e}$ and is equipped with a $*$-homomorphism $B \rightarrow M(A)$ that turns $A$ into a $(B, \Gamma)$-module. Such a $(B, \Gamma)$-algebra is proper if $B$ maps into $A$.

Given a $(B, \Gamma)$-algebra $A$ and $\gamma \in \Gamma$, we denote by $M(A)_{\gamma} \subseteq M(A)$ the space of all multipliers $T \in M(A)$ satisfying $T A_{\gamma^{\prime}} \subseteq A_{\gamma \gamma^{\prime}}$ and $A_{\gamma^{\prime}} T \subseteq A_{\gamma^{\prime} \gamma}$ for all $\gamma^{\prime} \in \Gamma$.

A morphism of $(B, \Gamma)$-algebras $A$ and $C$ is a non-degenerate, $B$-linear *-homomorphism $\pi: A \rightarrow M(C)$ satisfying $\pi\left(A_{\gamma}\right) \subseteq M(C)_{\gamma}$ for all $\gamma \in \Gamma$. Such a morphism is proper if it maps $A$ into $C$.

Using the extension of non-degenerate homomorphisms to multipliers, one defines the composition of morphisms and checks that $(B, \Gamma)$-algebras form a category.

The tensor product $B \otimes B$ is a $*$-algebra with local units and a natural action of $\Gamma \times \Gamma$. Replacing $(B, \Gamma)$ by $(B, \Gamma)^{\mathrm{ev}}:=(B \otimes B, \Gamma \times \Gamma)$ in the definition above, we obtain the category of all $(B, \Gamma)^{\mathrm{ev}}$-algebras.

Let $A$ be a $(B, \Gamma)^{\mathrm{ev}}$-algebra. We call an element $x \in A$ homogeneous and write $\partial_{x}=\gamma, \bar{\partial}_{x}=\gamma^{\prime}$ if $x \in A_{\gamma, \gamma^{\prime}}$ for some $\gamma, \gamma^{\prime} \in \Gamma$. Thus, $\partial_{x} \partial_{y}=\partial_{x y}, \bar{\partial}_{x} \bar{\partial}_{y}=\bar{\partial}_{x y}$ and $\partial_{x^{*}}=\partial_{x}^{-1}, \bar{\partial}_{x^{*}}=\bar{\partial}_{x}^{-1}$ for all homogeneous $x, y \in A$. Define $r=r_{A}: B \rightarrow$ $M(A)$ and $s=s_{A}: B \rightarrow M(A)$ by $r(b) a=(b \otimes 1) a$ and $s(b) a=(1 \otimes b) a$ for all $a \in A, b \in B$. We write ${ }_{r} A, A_{r},{ }_{s} A, A_{s}$ if we consider $A$ as a $B$-module via left or right multiplication via $r$ or $s$, respectively.

Clearly, $B$ is a $(B, \Gamma)$-algebra and $B \otimes B$ is a $(B, \Gamma)^{\mathrm{ev}}$-algebra with respect to the trivial gradings. Every $(B, \Gamma)$-algebra $A$ can be regarded as a $(B, \Gamma)^{\mathrm{ev}}$-algebra, where $A_{(\gamma, \gamma)}=A_{\gamma}$ and $A_{\left(\gamma, \gamma^{\prime}\right)}=0$ whenever $\gamma \neq \gamma^{\prime}$, and $\left(b \otimes b^{\prime}\right) a=b b^{\prime} a$ for all $b, b^{\prime} \in B, a \in A$. Conversely, every $(B, \Gamma)^{\mathrm{ev}}$-algebra $A$ can be considered as a $(B, \Gamma)$-algebra via $r: B \rightarrow M(A)$ and the grading given by $A_{\gamma}:=\bigoplus_{\gamma^{\prime}} A_{\gamma, \gamma^{\prime}}$, or via $s: B \rightarrow M(A)$ and the grading given by $A_{\gamma^{\prime}}:=\bigoplus_{\gamma} A_{\gamma, \gamma^{\prime}}$. We write $(A, r)$ and $(A, s)$, respectively, to denote the resulting $(B, \Gamma)$-algebras.

Denote by $B \rtimes \Gamma$ the crossed product for the action of $\Gamma$ on $B$, that is, the universal algebra containing $B$ and $\Gamma$ such that $e=1_{B}$ and $b \gamma \cdot b^{\prime} \gamma^{\prime}=b \gamma\left(b^{\prime}\right) \gamma \gamma^{\prime}$ for all $b, b^{\prime} \in B, \gamma, \gamma^{\prime} \in \Gamma$. This is a $(B, \Gamma)$-algebra with respect to the natural inclusion $B \rightarrow B \rtimes \Gamma$ and the involution and grading given by $(b \gamma)^{*}=\gamma^{-1} b^{*}$ and $(B \rtimes \Gamma)_{\gamma}=B \gamma$ for all $b \in B, \gamma \in \Gamma$. 
The fiber product of $(B, \Gamma)^{\mathrm{ev}}$-algebras $A$ and $C$ is defined as follows. The subalgebra

$$
A \stackrel{\Gamma}{\otimes} C:=\sum_{\gamma, \gamma^{\prime}, \gamma^{\prime \prime} \in \Gamma} A_{\gamma, \gamma^{\prime}} \otimes C_{\gamma^{\prime}, \gamma^{\prime \prime}} \subset A \otimes C
$$

is a $(B, \Gamma)^{\mathrm{ev}}$-algebra, where $(A \stackrel{\Gamma}{\otimes} C)_{\gamma, \gamma^{\prime \prime}}=\sum_{\gamma^{\prime}} A_{\gamma, \gamma^{\prime}} \otimes C_{\gamma^{\prime}, \gamma^{\prime \prime}}$ for all $\gamma, \gamma^{\prime \prime} \in \Gamma$ and $(r \times s)\left(b \otimes b^{\prime}\right)=r_{A}(b) \otimes s_{C}\left(b^{\prime}\right)$ for all $b, b^{\prime} \in B$. Let $I \subseteq M(A \stackrel{\Gamma}{\otimes} C)$ be the ideal generated by $\left\{s_{A}(b) \otimes 1-1 \otimes r_{C}(b): b \in B\right\}$. Then the quotient

$$
A \tilde{\otimes} C:=A \stackrel{\Gamma}{\otimes} C /(I(A \stackrel{\Gamma}{\otimes} C))
$$

is a $(B, \Gamma)^{\mathrm{ev}}$-algebra again, called the fiber product of $A$ and $C$. Write $a \tilde{\otimes} c$ for the image of an element $a \otimes c$ in $A \tilde{\otimes} C$.

The assignment $(A, C) \mapsto A \tilde{\otimes} C$ is functorial, associative and unital. Indeed, for all morphisms of $(B, \Gamma)^{\mathrm{ev}}$-algebras $\pi^{1}: A^{1} \rightarrow C^{1}, \pi^{2}: A^{2} \rightarrow C^{2}$, there exists a morphism

$$
\pi^{1} \tilde{\otimes} \pi^{2}: A^{1} \tilde{\otimes} A^{2} \rightarrow C^{1} \tilde{\otimes} C^{2}, \quad a_{1} \tilde{\otimes} a_{2} \mapsto \pi^{1}\left(a_{1}\right) \tilde{\otimes} \pi^{2}\left(a_{2}\right) ;
$$

for all $(B, \Gamma)^{\mathrm{ev}}$-algebras $A, C, D$, there exists an isomorphism

$$
(A \tilde{\otimes} C) \tilde{\otimes} D \rightarrow A \tilde{\otimes}(C \tilde{\otimes} D), \quad(a \tilde{\otimes} c) \tilde{\otimes} d \mapsto a \tilde{\otimes}(c \tilde{\otimes} d),
$$

and for each $(B, \Gamma)^{\mathrm{ev}}$-algebra $A$, there exist isomorphisms

$$
(B \rtimes \Gamma) \tilde{\otimes} A \rightarrow A, b \gamma \tilde{\otimes} a \mapsto r(b) a, \quad A \tilde{\otimes}(B \rtimes \Gamma) \rightarrow A, a \tilde{\otimes} b \gamma \mapsto s(b) a .
$$

These isomorphisms are compatible in a natural sense and endow the category of $(B, \Gamma)^{\mathrm{ev}}$-algebras with a monoidal structure. From now on, we shall use them without further notice.

The category of $(B, \Gamma)^{\mathrm{ev}}$-algebras carries automorphisms $(-)^{\mathrm{op}}$ and $(-)^{\mathrm{co}}$ such that for each $(B, \Gamma)$-algebra $A$ and each morphism $\phi: A \rightarrow C$, we have $A^{\text {co }}=A$ as an algebra, $A^{\mathrm{op}}$ is the opposite $*$-algebra of $A$, that is, the same vector space with the same involution and reversed multiplication, and

$$
\begin{aligned}
& \left(A^{\mathrm{op}}\right)_{\gamma, \gamma^{\prime}}=A_{\gamma^{-1}, \gamma^{\prime}-1} \text { for all } \gamma, \gamma^{\prime} \in \Gamma, \quad r_{A^{\mathrm{op}}}=r_{A}, \quad s_{A^{\mathrm{op}}}=s_{A}, \quad \phi^{\mathrm{op}}=\phi, \\
& \left(A^{\mathrm{co}}\right)_{\gamma, \gamma^{\prime}}=A_{\gamma^{\prime}, \gamma} \text { for all } \gamma, \gamma^{\prime} \in \Gamma, \quad r_{A^{\mathrm{co}}}=s_{A}, \quad s_{A^{\mathrm{co}}}=r_{A}, \quad \phi^{\mathrm{co}}=\phi .
\end{aligned}
$$

These automorphisms are involutive and commute, that is,

$$
(-)^{\mathrm{op}} \circ(-)^{\mathrm{op}}=\mathrm{id}, \quad(-)^{\mathrm{co}} \circ(-)^{\mathrm{co}}=\mathrm{id}, \quad(-)^{\mathrm{op}} \circ(-)^{\mathrm{co}}=(-)^{\mathrm{co}} \circ(-)^{\mathrm{op}} .
$$


Furthermore, they are compatible with the monoidal structure as follows. Given $(B, \Gamma)$-algebras $A, C$, there exist isomorphisms $(A \tilde{\otimes} C)^{\mathrm{op}} \rightarrow A^{\mathrm{op}} \tilde{\otimes} C^{\mathrm{op}}$ and $(A \tilde{\otimes} C)^{\mathrm{co}} \rightarrow C^{\mathrm{co}} \tilde{\otimes} A^{\mathrm{co}}$ given by $a \tilde{\otimes} c \mapsto a \tilde{\otimes} c$ and $a \tilde{\otimes} c \mapsto c \tilde{\otimes} a$, respectively. Moreover, $(B \rtimes \Gamma)^{\mathrm{co}}=B \rtimes \Gamma$, there exists an isomorphism $S^{B \rtimes \Gamma}: B \rtimes \Gamma \rightarrow$ $(B \rtimes \Gamma)^{\mathrm{op}}, b \gamma \mapsto \gamma^{-1} b$, and all of these isomorphisms and the isomorphisms in (2.2) and (2.3) are compatible in a natural sense.

2.3. Multiplier $(B, \Gamma)$-Hopf $*$-algebroids. We shall work with variants of the $\mathfrak{h}$-Hopf algebroids and $(B, \Gamma)$-Hopf $*$-algebroids considered in [7, 10] and [21], respectively, where the basis need no longer be unital. These variants consist of a $(B, \Gamma)^{\mathrm{ev}}$-algebra and a comultiplication, counit and antipode, which will be introduced one after the other. To quickly proceed to the main part of this article, we postulate all the usual properties of these maps as axioms and leave a study of the axiomatics for later.

Given a $(B, \Gamma)^{\mathrm{ev}}$-algebra $A$, we denote by $\tilde{M}(A \tilde{\otimes} A) \subseteq M(A \tilde{\otimes} A)$ the set of all $T \in M(A \tilde{\otimes} A)$ for which all products of the form

$$
T\left(x \tilde{\otimes} 1_{M(A)}\right), \quad\left(x \tilde{\otimes} 1_{M(A)}\right) T, \quad T\left(1_{M(A)} \tilde{\otimes} y\right), \quad\left(1_{M(A)} \tilde{\otimes} y\right) T
$$

where $x \in A_{\gamma, e}, y \in A_{e, \gamma}, \gamma \in \Gamma$, lie in $A \tilde{\otimes} A$. Evidently, $\tilde{M}(A \tilde{\otimes} A)$ is a *-subalgebra of $M(A \tilde{\otimes} A)$.

Definition 2.3.1. A comultiplication on a $(B, \Gamma)^{\mathrm{ev}}$-algebra $A$ is a morphism $\Delta$ from $A$ to $A \tilde{\otimes} A$ satisfying $\Delta(A) \subseteq \tilde{M}(A \tilde{\otimes} A)$ and $(\Delta \tilde{\otimes}$ id $) \circ \Delta=(\operatorname{id} \tilde{\otimes} \Delta) \circ \Delta$. A (proper) multiplier $(B, \Gamma)$-*-bialgebroid is a (proper) $(B, \Gamma)^{\mathrm{ev}}$-algebra with a comultiplication. A morphism of multiplier $(B, \Gamma)$ - $*$-bialgebroids $\left(A, \Delta_{A}\right)$, $\left(B, \Delta_{B}\right)$ is a morphism $\phi$ from $A$ to $B$ satisfying $\Delta_{B} \circ \phi=(\phi \tilde{\otimes} \phi) \circ \Delta_{A}$.

Let $(A, \Delta)$ be a multiplier $(B, \Gamma)$ - $*$-bialgebroid.

We shall need to form products of the form $\Delta(x)(1 \otimes y)$ or $(y \otimes 1) \Delta(x)$ when $\partial_{y} \neq e$ or $\bar{\partial}_{y} \neq e$, respectively, which are defined as follows. Let $x, y \in A$. The multiplication on $A \otimes A$ induces a canonical $A \tilde{\otimes} A-A \otimes A$-bimodule structure on ${ }_{s} A \underset{B}{\otimes} r A$ and a canonical $A \otimes A-A \tilde{\otimes} A$-bimodule structure on $A_{s} \underset{B}{\otimes} A_{r}$. Using the natural maps ${ }_{s} M(A) \otimes_{B} M(A) \rightarrow M\left({ }_{s} A \otimes_{B} A\right)$ and $M(A)_{s} \otimes_{B} M(A)_{r} \rightarrow M\left(A_{s} \otimes_{B} A_{r}\right)$, we define multipliers $1{\underset{B}{B}}_{y} y, x{\underset{B}{\otimes}}_{1} 1 \in M\left({ }_{s} A{\underset{B}{r}}_{r} A\right)$ and $x \underset{B}{\otimes} 1,1 \otimes_{B} y \in M\left(A_{s} \otimes_{B} A_{r}\right)$. Regarding $M\left({ }_{s} A \underset{B}{\otimes}{ }_{r} A\right)$ as an $M(A \tilde{\otimes} A)-M(A \otimes A)$-bimodule and $M\left(A_{s} \underset{B}{\otimes} A_{r}\right)$ as an $M(A \otimes A)-M(A \tilde{\otimes} A)$-bimodule (see $\S 2.1)$, we can then multiply these multipliers with $\Delta(x)$ or $\Delta(y)$, respectively. 
Lemma 2.3.2. The following linear maps are well defined:

$$
\begin{aligned}
& T_{1}: A_{s} \underset{B}{\otimes}{ }_{s} A \rightarrow{ }_{s} A \underset{B}{\otimes} A, \quad x \underset{B}{\otimes} y \mapsto \Delta(x)(1 \underset{B}{\otimes} y), \\
& T_{2}: A_{r} \underset{B}{\otimes} r A \rightarrow A_{s} \underset{B}{\otimes} A_{r}, \quad x \underset{B}{\otimes} y \mapsto(x \underset{B}{\otimes} 1) \Delta(y), \\
& T_{3}:{ }_{s} A \underset{B}{\otimes} A_{s} \rightarrow A_{s} \underset{B}{\otimes} A_{r}, \quad x \underset{B}{\otimes} y \mapsto(1 \underset{B}{\otimes} y) \Delta(x), \\
& T_{4}:{ }_{r} A \underset{B}{\otimes} A_{r} \rightarrow{ }_{s} A \underset{B}{\otimes}{ }_{r} A, \quad x \underset{B}{\otimes} y \mapsto \Delta(y)(x \underset{B}{\otimes} 1) .
\end{aligned}
$$

Proof. We only prove the assertion concerning $T_{1}$, the cases of $T_{2}, \ldots, T_{4}$ being similar. Using the explanations above, we obtain a linear map $A \otimes A \rightarrow M\left({ }_{s} A \otimes_{B} A\right)$, $x \otimes y \mapsto \Delta(x)(1 \underset{B}{\otimes} y)$. This map factorizes through the quotient map $A \otimes A \rightarrow$ $A_{s} \otimes_{B} A$ because $\Delta(x s(b))=\Delta(x)(1 \tilde{\otimes} s(b))$ for all $x \in A, b \in B$, and takes values

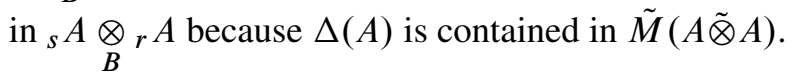

We adopt the Sweedler notation and write $\Delta(x)=\sum x_{(1)} \tilde{\otimes} x_{(2)}$ for $x \in A$. This notation requires extra care because $\Delta(x)$ need not lie in $A \tilde{\otimes} A$ but only in $\tilde{M}(A \tilde{\otimes} A)$, so that $x_{(1)}$ and $x_{(2)}$ do not simply represent elements of $A$. In this notation, the maps introduced above take the form

$$
\begin{array}{rlrl}
T_{1}: x \underset{B}{\otimes} y & \mapsto \sum x_{(1)} \underset{B}{\otimes} x_{(2)} y, & T_{2}: x \underset{B}{\otimes} y \mapsto \sum x y_{(1)} \underset{B}{\otimes} y_{(2)}, \\
T_{3}: x \underset{B}{\otimes} y \mapsto \sum x_{(1)} \underset{B}{\otimes} y x_{(2)}, & T_{4}: x \underset{B}{\otimes} y \mapsto \sum y_{(1)} x \underset{B}{\otimes} y_{(2)} .
\end{array}
$$

We shall almost exclusively use the Sweedler notation for products as above. A detailed explanation of this notation in the context of multiplier Hopf algebras is given in [30,32]. Apart from the fact that we use tensor products of $B$-modules instead of tensor products of vector spaces, this explanation carries over easily. As in the theory of (multiplier) Hopf algebras, we extend the Sweedler notation to iterated applications of $\Delta$, writing

$$
(\Delta \tilde{\otimes} \mathrm{id})(\Delta(x))=\sum x_{(1)} \tilde{\otimes} x_{(2)} \tilde{\otimes} x_{(3)}=(\mathrm{id} \tilde{\otimes} \Delta)(\Delta(x))
$$

for $x \in A$, and to iterated applications of the maps $T_{1}, \ldots, T_{4}$, writing, for example,

$$
\begin{aligned}
\left(T_{2} \underset{B}{\otimes} \mathrm{id}\right)\left(\left(\mathrm{id} \underset{B}{\otimes} T_{1}\right)(x \underset{B}{\otimes} y \underset{B}{\otimes} z)\right) & =\sum x y_{(1)} \underset{B}{\otimes} y_{(2)} \underset{B}{\otimes} y_{(3)} z \\
& =\left(\mathrm{id} \underset{B}{\otimes} T_{1}\right)\left(\left(T_{2} \underset{B}{\otimes} \mathrm{id}\right)(x \underset{B}{\otimes} y \underset{B}{\otimes} z)\right)
\end{aligned}
$$

for all $x, y, z \in A$.

Definition 2.3.3. A counit for a multiplier $(B, \Gamma)-*$-bialgebroid $(A, \Delta)$ is a proper morphism of $(B, \Gamma)^{\mathrm{ev}}$-algebras $\epsilon: A \rightarrow B \rtimes \Gamma$ satisfying $(\epsilon \tilde{\otimes}$ id $) \circ \Delta=\mathrm{id}_{A}=$ (id $\tilde{\otimes} \epsilon) \circ \Delta$. 
Let $(A, \Delta)$ be a multiplier $(B, \Gamma)$-*-bialgebroid with counit $\epsilon$. Using the linear maps

$$
\sharp: B \rtimes \Gamma \rightarrow B, \sum_{\gamma} b_{\gamma} \gamma \mapsto \sum_{\gamma} b_{\gamma}, \quad b: B \rtimes \Gamma \rightarrow B, \sum_{\gamma} \gamma b_{\gamma} \mapsto \sum_{\gamma} b_{\gamma},
$$

we define $\epsilon^{\sharp}, \epsilon^{\mathrm{b}}: A \rightarrow B$ by $\epsilon^{\sharp}:=\sharp \circ \epsilon$ and $\epsilon^{\mathrm{b}}:=\mathrm{b} \circ \epsilon$. Define $m_{r}: A_{r} \underset{B}{\otimes_{r} A \rightarrow A}$ and $m_{s}: A_{s} \underset{B}{\otimes} A \rightarrow A$ by $\sum_{i} x_{i} \underset{B}{\otimes} y_{i} \mapsto \sum_{i} x_{i} y_{i}$.

\section{Remarks 2.3.4.}

i) Clearly, $\epsilon\left(A_{\gamma, \gamma^{\prime}}\right) \subseteq(B \rtimes \Gamma)_{\gamma, \gamma^{\prime}}=0$ whenever $\gamma, \gamma^{\prime} \in \Gamma$ and $\gamma \neq \gamma^{\prime}$.

ii) If $\epsilon^{\prime}$ is a counit as well, then $\epsilon=\epsilon \circ\left(\right.$ id $\left.\tilde{\otimes} \epsilon^{\prime}\right) \circ \Delta=\epsilon^{\prime} \circ(\epsilon \tilde{\otimes}$ id $) \circ \Delta=\epsilon^{\prime}$.

iii) The condition $(\epsilon \tilde{\otimes}$ id $) \circ \Delta=\operatorname{id}_{A}=(\mathrm{id} \tilde{\otimes} \epsilon) \circ \Delta$ is equivalent to the relations

$$
\sum r\left(\epsilon^{\sharp}\left(x_{(1)}\right)\right) x_{(2)} y=x y=\sum x y_{(1)} s\left(\epsilon^{\mathrm{b}}\left(y_{(2)}\right)\right) \text { for all } x, y \in A,
$$

and hence to commutativity of the diagrams
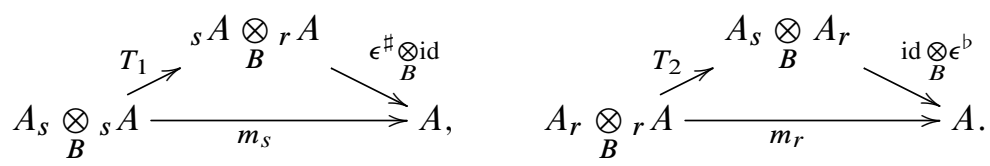

Furthermore, this condition is equivalent to the relations

$$
\sum x y_{(2)} r\left(\epsilon^{\mathrm{b}}\left(y_{(1)}\right)\right)=x y=\sum s\left(\epsilon^{\sharp}\left(x_{(2)}\right)\right) x_{(1)} y \quad \text { for all } x, y \in A .
$$

The definition of the antipode involves the isomorphism

$$
\sigma_{A, A}:(A \tilde{\otimes} A)^{\mathrm{co}, \mathrm{op}} \rightarrow A^{\mathrm{co}, \mathrm{op}} \tilde{\otimes} A^{\mathrm{co}, \mathrm{op}}, \quad x \tilde{\otimes} y \mapsto y \tilde{\otimes} x .
$$

Definition 2.3.5. An antipode for a multiplier $(B, \Gamma)$-*-bialgebroid $(A, \Delta)$ with counit $\epsilon$ is an isomorphism $S: A \rightarrow A^{\text {co,op }}$ of $(B, \Gamma)^{\mathrm{ev}}$-algebras that makes the following diagrams commute:

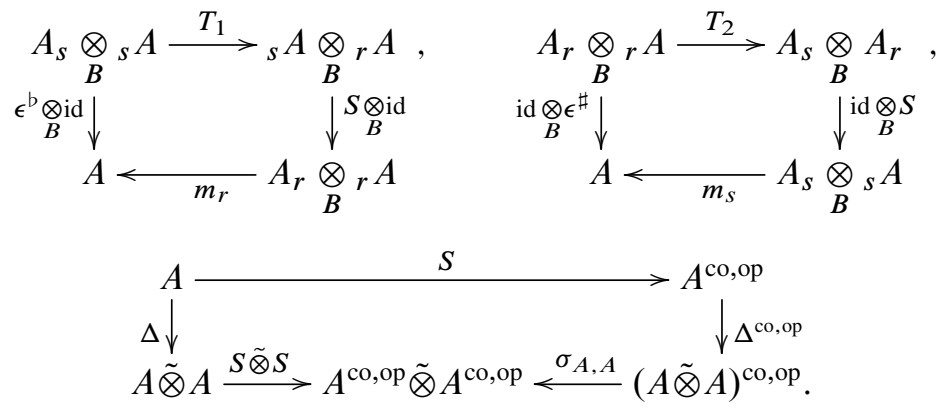

A multiplier $(B, \Gamma)$-Hopf $*$-algebroid is a multiplier $(B, \Gamma)$-*-bialgebroid with counit and antipode. 


\section{Examples 2.3.6.}

i) The tensor product $B \otimes B$ is a multiplier $(B, \Gamma)$-Hopf $*$-algebroid, where $\Delta\left(b \otimes b^{\prime}\right)=(b \otimes 1) \tilde{\otimes}\left(1 \otimes b^{\prime}\right), \epsilon\left(b \otimes b^{\prime}\right)=b b^{\prime}, S\left(b \otimes b^{\prime}\right)=b^{\prime} \otimes b$ for all $b, b^{\prime} \in B$.

ii) The crossed product $B \rtimes \Gamma$ is a multiplier $(B, \Gamma)$-Hopf $*$-algebroid, where $\Delta(b \gamma)=b \gamma \tilde{\otimes} \gamma=\gamma \tilde{\otimes} b \gamma, \epsilon=\mathrm{id}$ and $S(\gamma b)=b \gamma^{-1}$ for all $b \in B, \gamma \in \Gamma$.

Given an antipode $S$ on a multiplier $(B, \Gamma)$-*-bialgebroid $(A, \Delta)$ and an element $a \in A$, we shall henceforth always regard $S(a)$ as an element of $A$ and not of $A^{\mathrm{co}, \mathrm{op}}$.

Remarks 2.3.7. Let $(A, \Delta, \epsilon, S)$ be a multiplier $(B, \Gamma)$-Hopf $*$-algebroid.

i) In Sweedler notation, commutativity of the diagrams in Definition 2.3.5 amount to

$$
\begin{aligned}
& \sum S\left(x_{(1)}\right) x_{(2)} y=s\left(\epsilon^{b}(x)\right) y, \quad \sum x y_{(1)} S\left(y_{(2)}\right)=x r\left(\epsilon^{\sharp}(y)\right) \\
& \text { for all } x, y \in A \text {, } \\
& \sum S\left(x_{(1)}\right) \tilde{\otimes} S\left(x_{(2)}\right)=\sum S(x)_{(2)} \tilde{\otimes} S(x)_{(1)} \quad \text { for all } x \in A \text {. }
\end{aligned}
$$

ii) If $S^{\prime}$ is an antipode as well, then $S^{\prime}=S$ because for all $x, y, z \in A$,

$$
\begin{aligned}
x S(y) z=S\left(y S^{-1}(x)\right) z & =\sum S\left(s\left(\epsilon^{\sharp}\left(y_{(2)}\right)\right) y_{(1)} S^{-1}(x)\right) z \\
& =\sum S\left(y_{(2)} S^{-1}(x)\right) r\left(\epsilon^{\sharp}\left(y_{(2)}\right)\right) z \\
& =\sum S\left(y_{(1)} S^{-1}(x)\right) y_{(2)} S^{\prime}\left(S^{\prime-1}(z) y_{(3)}\right) \\
& =x S^{\prime}(y) z .
\end{aligned}
$$

For every multiplier $(B, \Gamma)$-Hopf $*$-algebroid, the maps $T_{1}, \ldots, T_{4}$ defined above are bijections.

Proposition 2.3.8. Let $(A, \Delta)$ be a multiplier $(B, \Gamma)$-*-bialgebroid. If $(A, \Delta)$ has a counit $\epsilon$ and an antipode $S$, then the maps $T_{1}, \ldots, T_{4}$ are bijective and for all $x, y \in A$,

$$
\begin{aligned}
& T_{1}^{-1}(x \underset{B}{\otimes} y)=\sum x_{(1)} \underset{B}{\otimes} S\left(S^{-1}(y) x_{(2)}\right), \\
& T_{2}^{-1}(x \underset{B}{\otimes} y)=\sum S\left(y_{(1)} S^{-1}(x)\right) \underset{B}{\otimes} y_{(2)}, \\
& T_{3}^{-1}(x \underset{B}{\otimes} y)=\sum x_{(1)} \underset{B}{\otimes} S^{-1}\left(x_{(2)} S(y)\right), \\
& T_{4}^{-1}(x \underset{B}{\otimes} y)=\sum S^{-1}\left(S(x) y_{(1)}\right) \underset{B}{\otimes} y_{(2)} .
\end{aligned}
$$


Proof. We only prove the assertion concerning $T_{1}$. One first checks that the formula given for $T_{1}^{-1}$ yields a well-defined map $T_{1}^{\prime}{ }_{s} A \underset{B}{\otimes}{ }_{r} A \rightarrow A_{s} \underset{B}{\otimes}{ }_{s} A$, and then that for all $x, y \in A$ and $u, v \in A_{e, e}$,

$$
\begin{aligned}
& (u \otimes v) \cdot\left(T_{1} \circ T_{1}^{\prime}\right)(x \underset{B}{\otimes} y)=\sum u x_{(1)} \underset{B}{\otimes} v x_{(2)} S\left(S^{-1}(y) x_{(3)}\right) \\
& =\sum u x_{(1)} \underset{B}{\otimes} v x_{(2)} S\left(x_{(3)}\right) y \\
& =\sum u x_{(1)} \underset{B}{\otimes} \operatorname{vr}\left(\epsilon^{\sharp}\left(x_{(2)}\right)\right) y \\
& =\sum u s\left(\epsilon^{\sharp}\left(x_{(2)}\right)\right) x_{(1)} \underset{B}{\otimes} v y=u x \underset{B}{\otimes} v y, \\
& (u \otimes v) \cdot\left(T_{1}^{\prime} \circ T_{1}\right)(x \underset{B}{\otimes} y)=\sum u x_{(1)} \underset{B}{\otimes} v S\left(S^{-1}\left(x_{(3)} y\right) x_{(2)}\right) \\
& =\sum u x_{(1)} \underset{B}{\otimes} v S\left(x_{(2)}\right) x_{(3)} y \\
& =\sum u x_{(1)} \underset{B}{\otimes} v s\left(\epsilon^{b}\left(x_{(2)}\right)\right) y \\
& =\sum u x_{(1)} s\left(\epsilon^{\mathrm{b}}\left(x_{(2)}\right)\right) \underset{B}{\otimes} v y=u x \underset{B}{\otimes} v y \text {. }
\end{aligned}
$$

2.4. Bi-measured multiplier $(B, \Gamma)$ - $*$-bialgebroids. We now introduce the main objects of this article - multiplier $(B, \Gamma)$-Hopf $*$-algebroids equipped with certain integrals. In $\S 3$, we shall construct completions of such objects in the form of measured quantum groupoids.

As on a groupoid, integration on a multiplier $(B, \Gamma)$-*-bialgebroid $(A, \Delta)$ proceeds in stages. First, one needs partial integrals $\phi, \psi: A \rightarrow B$ with suitable left or right invariance properties, and second a suitable weight $\mu: B \rightarrow \mathbb{C}$ that is compatible with the action of $\Gamma$. The results in [10] suggest that dynamical quantum groups that are compact in a suitable sense even possess a bi-invariant integral $h: A \rightarrow B \otimes B$ that can be obtained from a Peter-Weyl decomposition of $A$.

We first focus on the weight $\mu$ and the bi-integral $h$, and discuss left and right integrals in the next subsection.

Let us briefly recall some terminology. Let $C$ be a $*$-algebra with local units. A linear map $\mu: C \rightarrow \mathbb{C}$ is faithful if $\mu(C c)=0$ implies $c=0$, and positive if $\mu\left(c^{*} c\right) \geq 0$ for all $c \in C$. Assume that $\mu$ is positive. Then $\mu$ is $*$-linear, because positivity of $\mu\left((b+c)^{*}(b+c)\right)$ and $\mu\left((b+i c)^{*}(b+i c)\right)$ implies $\mu\left(b^{*} c\right)=\overline{\mu\left(c^{*} b\right)}$ for all $b, c \in C$, and faithful as soon as $\mu\left(c^{*} c\right) \neq 0$ whenever $c \neq 0$.

Definition 2.4.1. A weight for $(B, \Gamma)$ is a faithful, positive linear map $\mu: B \rightarrow \mathbb{C}$ that is quasi-invariant with respect to $\Gamma$ in the sense that for each $\gamma \in \Gamma$, there exists some $D_{\gamma} \in M(B)$ such that $\mu\left(\gamma\left(b D_{\gamma}\right)\right)=\mu(b)$ for all $b \in B$. 
Remark 2.4.2. Let $\mu$ be a weight for $(B, \Gamma)$. Then

i) each $D_{\gamma}$ is uniquely determined and self-adjoint,

ii) $D_{\gamma \gamma^{\prime}}=\gamma^{\prime-1}\left(D_{\gamma}\right) D_{\gamma^{\prime}}$ and $1=\gamma^{-1}\left(D_{\gamma^{-1}}\right) D_{\gamma}$ for all $\gamma, \gamma^{\prime} \in \Gamma$,

iii) $\left.\left.\mu\left(\gamma^{-1}(b) c\right)=\mu\left(b \gamma(c) D_{\gamma^{-1}}^{-1}\right)\right)\right)=\mu\left(b \gamma\left(c D_{\gamma}\right)\right)$ for all $b, c \in B, \gamma \in \Gamma$.

Indeed, i) and ii) follow easily from the fact that $\mu$ is faithful and the relations $\mu\left(\gamma\left(b D_{\gamma}^{*}\right)\right)=\overline{\mu\left(\gamma\left(D_{\gamma} b^{*}\right)\right)}=\overline{\mu\left(b^{*}\right)}=\mu(b)$ and $\mu\left(\gamma\left(\gamma^{\prime}\left(b D_{\gamma \gamma^{\prime}}\right)\right)\right)=\mu(b)=$ $\mu\left(\gamma^{\prime}\left(b D_{\gamma^{\prime}}\right)\right)=\mu\left(\gamma\left(\gamma^{\prime}\left(b D_{\gamma^{\prime}}\right) D_{\gamma}\right)\right)$.

We henceforth call the family $\left(D_{\gamma}\right)_{\gamma \in \Gamma}$ the Radon-Nikodym cocycle of $\mu$.

The following definition is inspired by the notion of a Haar functional introduced in [10].

Definition 2.4.3. A bi-integral on $(A, \Delta)$ is a morphism of $(B, \Gamma)^{\mathrm{ev}}$-modules $h: A \rightarrow$ $B \otimes B$ satisfying $\Delta(\operatorname{ker} h)\left(1 \tilde{\otimes} A_{e, e}\right) \subseteq \operatorname{ker} h \tilde{\otimes} A$ and $\Delta(\operatorname{ker} h)\left(A_{e, e} \tilde{\otimes} 1\right) \subseteq A \tilde{\otimes} \operatorname{ker} h$. If $(A, \Delta)$ is proper and $h\left(r(b) s\left(b^{\prime}\right)\right)=b \otimes b^{\prime}$ for all $b, b^{\prime} \in B$, we call such a biintegral normalized.

Lemma 2.4.4. Let $(A, \Delta)$ be proper and let $h$ be a normalized bi-integral on $(A, \Delta)$.

i) (id $\left.\tilde{\otimes} m_{B} \circ h\right) \circ \Delta=h=\left(m_{B} \circ h \tilde{\otimes}\right.$ id $) \circ \Delta$, where $m_{B}: B \otimes B \rightarrow B$ denotes the multiplication.

ii) If $h^{\prime}$ is a normalized bi-integral on $(A, \Delta)$, then $h^{\prime}=h$.

iii) If $(A, \Delta, \epsilon, S)$ is a proper multiplier $(B, \Gamma)$-Hopf $*$-algebroid, then $h \circ S=$ $\sigma_{B} \circ h$, where $\sigma_{B}: B \otimes B \rightarrow B \otimes B$ denotes the flip $b \otimes c \mapsto c \otimes b$.

Proof. i) We only prove the first equation. Let $\omega:(A, r) \rightarrow B$ be a morphism of $(B, \Gamma)$-modules sending $I:=\operatorname{ker} h$ to 0 . Then

$$
(\operatorname{id} \tilde{\otimes} \omega)(\Delta(I)) A_{e, e}=(\operatorname{id} \underset{B}{\otimes} \omega)\left(\Delta(I)\left(A_{e, e} \tilde{\otimes} 1\right)\right) \subseteq(\operatorname{id} \underset{B}{\otimes} \omega)(A \underset{B}{\otimes} I)=0
$$

and hence $(\operatorname{id} \tilde{\otimes} \omega)(\Delta(I))=0$. Moreover, if $b, b^{\prime}, b^{\prime \prime} \in B$ and $u \in A_{e, e}$, then

$$
\begin{aligned}
(\mathrm{id} \tilde{\otimes} \omega)\left(\Delta\left(r(b) s\left(b^{\prime}\right)\right)\right) s\left(b^{\prime \prime}\right) u & =\left(\underset{B}{\operatorname{id} \omega}\left(r(b) s\left(b^{\prime \prime}\right) u \underset{B}{\otimes} s\left(b^{\prime}\right)\right)\right. \\
& =r(b) s\left(\omega\left(s\left(b^{\prime}\right) r\left(b^{\prime \prime}\right)\right)\right) u .
\end{aligned}
$$

For $\omega=m_{B} \circ h$, these calculations imply for all $a \in I$ and $b, b^{\prime} \in B$

$$
\begin{gathered}
\left(\operatorname{id} \tilde{\otimes} m_{B} \circ h\right)(\Delta(a))=0=h(a), \\
\left(\operatorname{id} \tilde{\otimes} m_{B} \circ h\right)\left(\Delta\left(r(b) s\left(b^{\prime}\right)\right)\right)=r(b) s\left(b^{\prime}\right)=h\left(r(b) s\left(b^{\prime}\right)\right) .
\end{gathered}
$$

Since $A=I+r(B) s(B)$, we can conclude (id $\left.\tilde{\otimes} m_{B} \circ h\right) \circ \Delta=h$.

ii) Let $x \in \operatorname{ker} h$ and choose $u, u^{\prime} \in B \otimes B$ such that $u\left(1 \otimes m_{B}\left(u^{\prime}\right)\right) h^{\prime}(x)=$ $h^{\prime}(x)$. Then

$$
h^{\prime}(x)=h\left(u h^{\prime}(x) s\left(m_{B}\left(u^{\prime}\right)\right)\right)=\sum h\left(u x_{(1)} s\left(m_{B}\left(h^{\prime}\left(x_{(2)} u^{\prime}\right)\right)\right)\right)=0
$$


because $\sum u x_{(1)} \otimes x_{(2)} u^{\prime} \in u(\operatorname{ker} h) \underset{B}{\otimes} A$. Thus, $\operatorname{ker} h \subseteq \operatorname{ker} h^{\prime}$. Since $h$ and $h^{\prime}$ are normalized and $\operatorname{ker} h+B \otimes B=A$, we can conclude $h=h^{\prime}$.

iii) One easily verifies that $\sigma_{B} \circ h \circ S$ is a normalized bi-integral. By ii), it equals $h$.

Definition 2.4.5. A proper multiplier $(B, \Gamma)$-*-bialgebroid $(A, \Delta)$ is bi-measured if it is equipped with a normalized bi-integral $h: A \rightarrow B \otimes B$ and a weight $\mu$ for $(B, \Gamma)$ such that $v:=(\mu \otimes \mu) \circ h$ is faithful and positive.

Remark 2.4.6. Given a bi-measured proper multiplier $(B, \Gamma)$-Hopf $*$-algebroid as above, $h$ is evidently faithful, and also $*$-linear. To see this, note that $(\mu \otimes$ $\mu)\left(h\left(a^{*}\right)(b \otimes c)\right)=v\left(a^{*} r(b) s(c)\right)=\overline{v\left(s\left(c^{*}\right) r\left(b^{*}\right) a\right)}=\overline{(\mu \otimes \mu)\left((b \otimes c)^{*} h(a)\right)}=$ $(\mu \otimes \mu)\left(h(a)^{*}(b \otimes c)\right)$ for all $a \in A, b, c \in B$.

2.5. Left and right integrals. For large parts of this article, the multiplier $(B, \Gamma)$ Hopf $*$-algebroids under consideration need not be equipped with a bi-integral, but only with left and right integrals $\phi, \psi$. The definition of these integrals involves slice maps of the following form.

Let $(A, \Delta)$ be a multiplier $(B, \Gamma)$ - $*$-bialgebroid and let $\phi:(A, r) \rightarrow B$ be a morphism of $(B, \Gamma)$-modules. Then there exists a unique linear map id $\tilde{\otimes} \phi: \tilde{M}(A \tilde{\otimes} A) \rightarrow$ $M(A)$ such that

$$
((\operatorname{id} \tilde{\otimes} \phi)(T)) a=(\operatorname{id} \underset{B}{\otimes} \phi)(T(a \otimes 1)), \quad a((\operatorname{id} \tilde{\otimes} \phi)(T))=(\operatorname{id} \underset{B}{\otimes} \phi)((a \otimes 1) T)
$$

for all $T \in \tilde{M}(A \tilde{\otimes} A)$ and $a \in A$, where we regard $T(a \otimes 1)$ and $(a \otimes 1) T$ as elements of ${ }_{s} A \underset{B}{\otimes}{ }_{r} A$ and $A_{s} \underset{B}{\otimes} A_{r}$, respectively. In the case $T=\Delta(x)$ for some $x \in A$,

$$
(\mathrm{id} \tilde{\otimes} \phi)(\Delta(x)) a=\sum s\left(\phi\left(x_{(2)}\right)\right) x_{(1)} a, \quad a(\mathrm{id} \tilde{\otimes} \phi)(\Delta(x))=\sum a x_{(1)} s\left(\phi\left(x_{(2)}\right)\right) .
$$

Likewise, every morphism $\psi:(A, s) \rightarrow B$ yields a slice map $\psi \tilde{\otimes}$ id: $\tilde{M}(A \tilde{\otimes} A) \rightarrow$ $M(A)$.

Definition 2.5.1. A left integral on $(A, \Delta)$ is a morphism $\phi:(A, r) \rightarrow B$ satisfying (id $\tilde{\otimes} \phi) \circ \Delta=r \circ \phi$. A right integral on $(A, \Delta)$ is a morphism $\psi:(A, s) \rightarrow B$ satisfying $(\psi \tilde{\otimes}$ id $) \circ \Delta=s \circ \psi$.

\section{Remarks 2.5.2.}

i) In Sweedler notation, the invariance conditions on $\phi$ and $\psi$ become

$$
\sum s\left(\phi\left(x_{(2)}\right)\right) x_{(1)} a=r(\phi(x)) a, \quad \sum a x_{(2)} r\left(\psi\left(x_{(1)}\right)\right)=a s(\psi(x))
$$

for all $a, x \in A$. 
ii) If $(A, \Delta, \epsilon, S)$ is a $(B, \Gamma)$-Hopf $*$-algebroid, then the map $\phi \mapsto \phi \circ S$ gives a bijection between left and right integrals on $(A, \Delta)$. This follows easily from (2.7).

iii) If $\phi$ is a left integral, then $\phi(-s(b))$ is also a left integral for each $b \in B$. Likewise, if $\psi$ is a right integral, then also $\psi(-r(b))$ is a right integral for each $b \in B$.

We shall frequently use the following strong invariance relations:

Proposition 2.5.3. Assume that $(A, \Delta, \epsilon, S)$ is a $(B, \Gamma)$-Hopf $*$-algebroid. Then

i) $(\mathrm{id} \underset{B}{\otimes} \phi)((1 \tilde{\otimes} z) \Delta(x))=S((\operatorname{id} \underset{B}{\otimes} \phi)(\Delta(z)(1 \tilde{\otimes} x)))$ for every left integral $\phi$ and all $x, z \in A$;

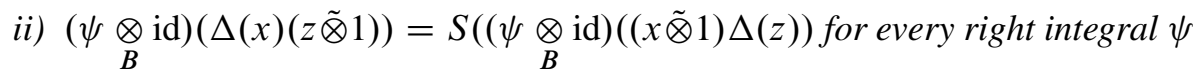
and all $x, z \in A$.

Proof. Using Sweedler notation, we calculate

$$
\begin{aligned}
\sum x_{(1)} s\left(\phi\left(z x_{(2)}\right)\right) & =\sum x_{(1)} s\left(\phi\left(z_{(2)} r\left(\epsilon^{b}\left(z_{(1)}\right)\right) x_{(2)}\right)\right) \\
& =\sum s\left(\epsilon^{b}\left(z_{(1)}\right)\right) x_{(1)} s\left(\phi\left(z_{(2)} x_{(2)}\right)\right) \\
& =\sum S\left(z_{(1)}\right) z_{(2)} x_{(1)} s\left(\phi\left(z_{(3)} x_{(2)}\right)\right)=\sum S\left(z_{(1)}\right) r\left(\phi\left(z_{(2)} x\right)\right)
\end{aligned}
$$

and

$$
\begin{aligned}
\sum r\left(\psi\left(x_{(1)} z\right)\right) x_{(2)} & =\sum r\left(\psi\left(x_{(1)} s\left(\epsilon^{\sharp}\left(z_{(2)}\right)\right) z_{(1)}\right)\right) x_{(2)} \\
& =\sum r\left(\psi\left(x_{(1)} z_{(1)}\right)\right) x_{(2)} r\left(\epsilon^{\sharp}\left(z_{(2)}\right)\right) \\
& =\sum r\left(\psi\left(x_{(1)} z_{(1)}\right)\right) x_{(2)} z_{(2)} S\left(z_{(3)}\right)=s\left(\psi\left(x z_{(1)}\right)\right) S\left(z_{(2)}\right) .
\end{aligned}
$$

Normalized bi-integrals yield left and right integrals as follows:

Lemma 2.5.4. Assume that $(A, \Delta)$ is proper, $h$ is a normalized bi-integral on $(A, \Delta)$, and $\mu: B \rightarrow \mathbb{C}$ is linear. Then $\phi:=(\mathrm{id} \otimes \mu) \circ h$ and $\psi:=(\mu \otimes \mathrm{id}) \circ h$ are a left and a right integral, respectively, and $\phi \circ S^{ \pm 1}=\psi$.

Proof. Repeating the proof of Lemma 2.4.4 i) with $\omega:=\phi=($ id $\otimes \mu) \circ h$, we find

$$
\begin{gathered}
(\operatorname{id} \tilde{\otimes} \phi)(\Delta(a))=0=r(\phi(a)), \\
(\operatorname{id} \tilde{\otimes} \phi)\left(\Delta\left(r(b) s\left(b^{\prime}\right)\right)\right)=r\left(b \mu\left(b^{\prime}\right)\right)=\phi\left(r(b) s\left(b^{\prime}\right)\right)
\end{gathered}
$$

for all $a \in \operatorname{ker} h$ and $b, b^{\prime} \in B$. Since $A=(\operatorname{ker} h)+r(B) s(B)$, we can conclude (id $\tilde{\otimes} \phi) \circ \Delta=r \circ \phi$. The assertion on $\psi$ follows similarly, and the last equation follows from Lemma 2.4.4 iii). 
2.6. Measured multiplier $(B, \Gamma)$-*-bialgebroids. Much of the ensuing material applies not only to bi-measured proper multiplier $(B, \Gamma)$-Hopf $*$-algebroids but also to the following class of objects.

Definition 2.6.1. A multiplier $(B, \Gamma)$-*-bialgebroid $(A, \Delta)$ is measured if it is equipped with a left integral $\phi$, a right integral $\psi$, and a weight $\mu$ for $(B, \Gamma)$ such that $v:=\mu \circ \phi$ and $v^{-1}:=\mu \circ \psi$ are faithful, positive, and coincide, and $\psi(A)=B=\phi(A)$.

\section{Remarks 2.6.2.}

i) Given a measured multiplier $(B, \Gamma)$-Hopf $*$-algebroid as above, the maps $\phi$ and $\psi$ are $*$-linear. This can be seen from a similar argument as in Remark 2.4.6.

ii) If ( $A, \Delta, \epsilon, S, h, \mu)$ is a bi-measured proper multiplier $(B, \Gamma)$-Hopf $*$-algebroid and $\phi=(\mathrm{id} \otimes \mu) \circ h$ and $\psi=(\mu \otimes \mathrm{id}) \circ h$, then $(A, \Delta, \epsilon, S, \phi, \psi, \mu)$ is a measured multiplier $(B, \Gamma)$-Hopf $*$-algebroid by Lemma 2.5.4. In that case, $\phi \circ S^{ \pm 1}=\psi$ and $v \circ S=v$ by Lemma 2.4.4 iii).

Till the end of this subsection, let $(A, \Delta, \epsilon, S, \phi, \psi, \mu)$ be a measured multiplier $(B, \Gamma)$-Hopf $*$-algebroid and let $\left(D_{\gamma}\right)_{\gamma}$ be the Radon-Nikodym cocycle for $\mu$. Define $D, \bar{D}: A \rightarrow A$ by

$$
D(a)=r\left(D_{\partial_{a}^{-1}}\right) a=\operatorname{ar}\left(D_{\partial_{a}}^{-1}\right), \quad \bar{D}(a)=s\left(D_{\bar{\partial}_{a}^{-1}}\right) a=\operatorname{as}\left(D_{\bar{\partial}_{a}}^{-1}\right)
$$

for all homogeneous $a \in A$.

Lemma 2.6.3. $D$ and $\bar{D}$ both are algebra and $(B, \Gamma)^{\mathrm{ev}}$-module automorphisms of $A$, and satisfy

$$
\begin{gathered}
(D \tilde{\otimes} \mathrm{id}) \circ \Delta=\Delta \circ D, \quad(\operatorname{id} \tilde{\otimes} \bar{D}) \circ \Delta=\Delta \circ \bar{D}, \quad(\bar{D} \tilde{\otimes} \mathrm{id}) \circ \Delta=(\operatorname{id} \tilde{\otimes} D) \circ \Delta, \\
D \circ \bar{D}=\bar{D} \circ D, \quad S \circ D=\bar{D}^{-1} \circ S, \quad S \circ \bar{D}=D^{-1} \circ S, \\
* \circ D=D^{-1} \circ *, \quad * \circ \bar{D}=\bar{D}^{-1} \circ * .
\end{gathered}
$$

Proof. The maps $D$ and $\bar{D}$ are bijective because $D_{\gamma}$ is invertible for each $\gamma \in \Gamma$. The remaining assertions follow from straightforward calculations, for example,

$$
\begin{gathered}
D(x y)=r\left(D_{\partial_{x y}^{-1}}\right) x y=r\left(D_{\partial_{x}^{-1}} \partial_{x}\left(D_{\partial_{y}^{-1}}\right)\right) x y=r\left(D_{\partial_{x}^{-1}}\right) x r\left(D_{\partial_{y}^{-1}}\right) y=D(x) D(y) \\
S(D(x))=S\left(r\left(D_{\partial_{x}}^{-1}\right) x\right)=S(x) S\left(D_{\bar{\partial}_{S(x)}}\right)=\bar{D}^{-1}(S(x)) \\
D(x)^{*}=x^{*} r\left(D_{\partial_{x}^{-1}}^{*}\right)=x^{*} r\left(D_{\partial_{x^{*}}}\right)=D^{-1}\left(x^{*}\right) \quad \text { for all } x, y \in A
\end{gathered}
$$

Lemma 2.6.4. Let $\omega \in\{\phi, \psi, v\}$.

i) $\omega\left(A_{\gamma, \gamma^{\prime}}\right)=0$ whenever $\left(\gamma, \gamma^{\prime}\right) \neq(e, e)$.

ii) $\omega\left(r(b) s\left(b^{\prime}\right) a\right)=\omega\left(\operatorname{ar}(b) s\left(b^{\prime}\right)\right)$ for all $a \in A, b, b^{\prime} \in B$. 
iii) $\omega\left(D(a) a^{\prime}\right)=\omega\left(a D^{-1}\left(a^{\prime}\right)\right)$ and $\omega\left(\bar{D}(a) a^{\prime}\right)=\omega\left(a \bar{D}^{-1}\left(a^{\prime}\right)\right)$ for all $a, a^{\prime} \in$ $A$.

Proof. i) For $\omega=v$, the assertion follows from the relation $\operatorname{ker} \phi+\operatorname{ker} \psi \subseteq \operatorname{ker} v$. To obtain the assertion for $\omega=\phi, \psi$, use the fact that $\mu$ is faithful.

ii) Let $a \in A$ and $b, b^{\prime} \in B$. Then $v(r(b) a)=\mu(b \phi(a))=\mu(\phi(a) b)=$ $v(\operatorname{ar}(b))$ and similarly $v\left(s\left(b^{\prime}\right) a\right)=v\left(a s\left(b^{\prime}\right)\right)$. To obtain the assertion for $\omega=\phi, \psi$, use the fact that $\mu$ is faithful again.

iii) This follows immediately from equation (2.9) and i).

We shall now show that $v=\mu \circ \phi$ has a modular automorphism and thus satisfies an algebraic variant of the KMS-condition. Let us briefly recall this concept.

Let $C$ be a $*$-algebra with local units and a faithful, positive, linear map $\omega: C \rightarrow \mathbb{C}$. A modular automorphism for $\omega$ is a bijection $\theta_{\omega}: C \rightarrow C$ satisfying $\omega\left(c c^{\prime}\right)=\omega\left(c^{\prime} \theta_{\omega}(c)\right)$ for all $c, c^{\prime} \in C$. If it exists, a modular automorphism $\theta_{\omega}$ for $\omega$ is uniquely determined, an algebra automorphism, and satisfies $\omega \circ \theta_{\omega}=\omega$ and $\theta_{\omega} \circ * \circ \theta_{\omega} \circ *=\mathrm{id}_{C}$. This follows easily from the relations

$$
\begin{aligned}
\omega\left(z \theta_{\omega}(x y)\right) & =\omega(x y z)=\omega\left(y z \theta_{\omega}(x)\right)=\omega\left(z \theta_{\omega}(x) \theta_{\omega}(y)\right), \\
\omega(y x) & =\overline{\omega\left(x^{*} y^{*}\right)}=\overline{\omega\left(y^{*} \theta_{\omega}\left(x^{*}\right)\right)}=\omega\left(\theta_{\omega}\left(x^{*}\right)^{*} y\right)=\omega\left(y \theta_{\omega}\left(\theta_{\omega}\left(x^{*}\right)^{*}\right)\right),
\end{aligned}
$$

where $x, y, z \in C$.

As before, let $(A, \Delta, \epsilon, S, \phi, \psi, \mu)$ be a measured multiplier $(B, \Gamma)$-Hopf $*$ algebroid.

Theorem 2.6.5. Let $(A, \Delta, \epsilon, S, \phi, \psi, \mu)$ be a measured multiplier $(B, \Gamma)$-Hopf $*-$ algebroid and let $v=\mu \circ \phi=\mu \circ \psi$.

i) There exists a modular automorphism $\theta$ for $v$.

ii) $\theta$ is a $(B, \Gamma)^{\mathrm{ev}}$-module automorphism of $A$.

iii) If $v \circ S=v$, then $\theta \circ S=S \circ \theta^{-1}$.

Proof. i) The proof repeatedly uses strong invariance of $\phi$ and $\psi$, and closely follows [31], where the corresponding result was obtained for multiplier Hopf algebras. We proceed in three steps. 
Step 1. Repeatedly using Remark 2.4.2 iii), we find that for all homogeneous $x, x^{\prime}, y, y^{\prime} \in A$,

$$
\begin{aligned}
\bar{\partial}_{x^{\prime}}=\bar{\partial}_{y^{\prime}}^{-1} \quad \Rightarrow \quad v^{-1}\left(y s\left(\psi\left(x x^{\prime}\right)\right) y^{\prime}\right) & =\mu\left(\psi\left(y y^{\prime}\right) \bar{\partial}_{y^{\prime}}^{-1}\left(\psi\left(x x^{\prime}\right)\right)\right) \\
& =\mu\left(\psi\left(x x^{\prime}\right) \bar{\partial}_{y^{\prime}}\left(\psi\left(y y^{\prime}\right) D_{\bar{\partial}_{y^{\prime}}}\right)\right) \\
& =v^{-1}\left(x s\left(\psi\left(y y^{\prime}\right)\right) \bar{D}\left(x^{\prime}\right)\right), \\
\bar{\partial}_{x}=\partial_{y^{\prime}} \quad \Rightarrow \quad v\left(y r\left(\psi\left(x x^{\prime}\right)\right) y^{\prime}\right) & =\mu\left(\phi\left(y y^{\prime}\right) \partial_{y^{\prime}}^{-1}\left(\psi\left(x x^{\prime}\right)\right)\right) \\
& =\mu\left(\partial_{y^{\prime}}\left(\phi\left(y y^{\prime}\right) D_{\partial_{y^{\prime}}}\right) \psi\left(x x^{\prime}\right)\right) \\
& =v^{-1}\left(x s\left(\phi\left(D(y) y^{\prime}\right)\right) x^{\prime}\right), \\
\partial_{x}=\partial_{y}^{-1} \Rightarrow \quad v\left(y r\left(\phi\left(x x^{\prime}\right)\right) y^{\prime}\right) & =\mu\left(\partial_{y}\left(\phi\left(x x^{\prime}\right)\right) \phi\left(y y^{\prime}\right)\right) \\
& =\mu\left(\partial_{y}^{-1}\left(\phi\left(y y^{\prime}\right) D_{\partial_{y}^{-1}}\right) \phi\left(x x^{\prime}\right)\right) \\
& =v\left(x r\left(\phi\left(D(y) y^{\prime}\right)\right) x^{\prime}\right) .
\end{aligned}
$$

Step 2. Let $c, d \in A$ be homogeneous and let

$a=\sum \bar{D}\left(s\left(\psi\left(d S\left(c_{(2)}\right)\right)\right) c_{(1)}\right) \in A, \quad a^{\prime}=\sum d_{(2)} r\left(\phi\left(D\left(S\left(d_{(1)}\right)\right) \bar{D}(c)\right)\right) \in A$.

Then the equations above and Proposition 2.5.3 imply

$$
\begin{aligned}
v(z a) & =\sum v\left(z \bar{D}\left(s\left(\psi\left(d S\left(c_{(2)}\right)\right)\right) c_{(1)}\right)\right) & & \\
& =\sum v\left(d s\left(\psi\left(z c_{(1)}\right)\right) S\left(c_{(2)}\right)\right) & & \text { (Equation (2.10)) } \\
& =\sum v\left(d r\left(\psi\left(z_{(1)} c\right)\right) z_{(2)}\right) & & \text { (Proposition 2.5.3) } \\
& =\sum v\left(z_{(1)} s\left(\phi\left(D(d) z_{(2)}\right)\right) c\right) & & \text { (Equation (2.11)) } \\
& =\sum v\left(S\left(D\left(d_{(1)}\right)\right) r\left(\phi\left(d_{(2)} z\right)\right) c\right) & & \text { (Proposition 2.5.3) } \\
& =\sum v\left(S\left(d_{(1)}\right) r\left(\phi\left(d_{(2)}\right)\right) \bar{D}(c)\right) & & \text { (use } S \circ D=\bar{D}^{-1} \circ S \\
& =\sum v\left(d_{(2)} r\left(\phi\left(D\left(S\left(d_{(1)}\right)\right) \bar{D}(c)\right)\right) z\right)=v\left(a^{\prime} z\right) . & & \text { (Equation 2.12) }
\end{aligned}
$$

Step 3. Using bijectivity of the maps $\bar{D}, S, T_{1}$ and the relation $\langle s(\psi(A)) A\rangle=A$, one finds that all elements of the form like $a$ in (2.13) span $A$. A similar argument shows that the same is true for elements of the form like $a^{\prime}$. Hence, there exists a bijection $\theta: A \rightarrow A$ such that $v(a z)=v(z \theta(a))$ for all $a \in A$, and uniqueness of such a bijection follows from faithfulness of $\nu$.

ii) We first show that $\theta$ respects the grading. Let $c, d \in A$ be homogeneous. Then the element $a$ in (2.13) is homogeneous as well, with grading given by $\partial_{a}=\partial_{c}$ and 
$\bar{\partial}_{a}=\bar{\partial}_{d}$ because $\psi\left(d S\left(c_{(2)}\right)\right)=0$ unless $\bar{\partial}_{d}=\partial_{c_{(2)}}=\bar{\partial}_{c_{(1)}}$, and similarly $a^{\prime}$ in (2.13) is homogeneous with the same degree like $a$. To see that $\theta$ is $B \otimes B$ linear, use the relation $v\left(y \theta\left(r(b) s\left(b^{\prime}\right) x\right)\right)=v\left(r(b) s\left(b^{\prime}\right) x y\right)=v\left(x y r(b) s\left(b^{\prime}\right)\right)=$ $v\left(y r(b) s\left(b^{\prime}\right) \theta(x)\right)$, where $x, y \in A$ and $b, b^{\prime} \in B$, and faithfulness of $v$.

iii) If $v \circ S=v$, then we have $v(y \theta(S(x)))=v(S(x) y)=v\left(S^{-1}(y) x\right)=$ $v\left(\theta^{-1}(x) S^{-1}(y)\right)=v\left(y S\left(\theta^{-1}(x)\right)\right)$ for all $x, y \in A$.

Define $\theta_{D}, \theta_{\bar{D}}, \theta_{D, \bar{D}}: A \rightarrow A$ by

$$
\begin{aligned}
\theta_{D} & :=\theta \circ D^{-1}=D^{-1} \circ \theta, \\
\theta_{\bar{D}} & :=\theta \circ \bar{D}^{-1}=\bar{D}^{-1} \circ \theta, \\
\theta_{D, \bar{D}} & :=\theta \circ D^{-1} \circ \bar{D}^{-1} .
\end{aligned}
$$

Proposition 2.6.6. Let $x, y \in A$ be homogeneous. Then

i) $\phi \circ \theta=\phi$ and $\phi(x y)=\partial_{x}\left(\phi\left(y \theta_{D}(x)\right)\right)$;

ii) $\psi \circ \theta=\psi$ and $\psi(x y)=\bar{\partial}_{x}\left(\psi\left(y \theta_{\bar{D}}(x)\right)\right)$;

iii) $h \circ \theta=h$ and $h(x y)=\left(\partial_{x} \otimes \bar{\partial}_{x}\right)\left(h\left(y \theta_{D, \bar{D}}(x)\right)\right)$ if $h$ is a bi-invariant integral and $v=(\mu \otimes \mu) \circ h$.

Proof. Assertion i) follows from the fact that $\mu$ is faithful and that for all homogeneous $x, y \in A$ and all $b \in B$,

$$
\begin{aligned}
\mu(b \phi(\theta(x)))=v(r(b) \theta(x)) & =v(\theta(r(b) x))=v(r(b) x)=\mu(b \phi(x)), \\
\mu(b \phi(y \theta(x)))=v(r(b) y \theta(x)) & =v(x r(b) y) \\
& =v\left(r\left(\partial_{x}\left(b D_{\partial_{x}}\right)\right) x r\left(D_{\partial_{x}}^{-1}\right) y\right) \\
& =\mu\left(\partial_{x}\left(b D_{\partial_{x}}\right) \phi(D(x) y)\right)=\mu\left(b \partial_{x}^{-1}(\phi(D(x) y))\right) .
\end{aligned}
$$

Assertions ii) and iii) follow similarly.

Recall that a $B$-module $N$ is called flat if the functor $N \underset{B}{\otimes}$ - on the category of $B$-modules is exact or, equivalently, preserves injectivity of morphisms.

Proposition 2.6.7. Assume that $A_{S}$ is a flat B-module. Then $\Delta \circ \theta_{D}=\left(S^{2} \tilde{\otimes} \theta_{D}\right) \circ \Delta$. 
Proof. Let $x, y \in A$ be homogeneous. Using Sweedler notation, we calculate

$$
\begin{aligned}
\sum \theta_{D}(x)_{(1)} s\left(\phi\left(y \theta_{D}(x)_{(2)}\right)\right) & =\sum S\left(s\left(\phi\left(y_{(2)} \theta_{D}(x)\right)\right) y_{(1)}\right) \\
& =\sum S\left(s\left(\partial_{x}^{-1}\left(\phi\left(x y_{(2)}\right)\right)\right) y_{(1)}\right) \\
& =\sum S\left(y_{(1)} s\left(\phi\left(x y_{(2)}\right)\right)\right) \\
& =\sum S^{2}\left(s\left(\phi\left(x_{(2)} y\right)\right) x_{(1)}\right) \\
& =\sum S^{2}\left(s\left(\partial_{x_{(2)}}\left(\phi\left(y \theta_{D}\left(x_{(2)}\right)\right)\right)\right) x_{(1)}\right) \\
& =\sum S^{2}\left(x_{(1)}\right) s\left(\phi\left(y \theta_{D}\left(x_{(2)}\right)\right)\right) .
\end{aligned}
$$

Since $A_{s}$ is a flat $B$-module and maps of the form $a \mapsto \phi(y a)$, where $y \in A$ is homogeneous, separate the points of $A$, we can conclude

$$
\sum \theta_{D}(x)_{(1)} \tilde{\otimes} \theta_{D}(x)_{(2)}=\sum S^{2}\left(x_{(1)}\right) \tilde{\otimes} \theta_{D}\left(x_{(2)}\right) .
$$

2.7. The dual $*$-algebra. Let $(A, \Delta, \epsilon, S, \phi, \psi, \mu)$ be a measured multiplier $(B, \Gamma)$-Hopf $*$-algebroid. Denote by $M(A)^{\prime}$ the dual vector space of $M(A)$ and let

$$
\hat{A}:=\{v(x-): x \in A\} \subseteq M(A)^{\prime} .
$$

Then $\hat{A}=\{v(-x): x \in A\}$ by Theorem 2.6.5 and for each $\omega \in \hat{A}$, there exist unique $B$-module maps ${ }_{r} \omega:{ }_{r} M(A) \rightarrow B, \omega r: M(A)_{r} \rightarrow B,{ }_{s} \omega:{ }_{s} M(A) \rightarrow B$, $\omega_{s}: M(A)_{s} \rightarrow B$ whose compositions with $\mu$ are equal to $\omega$, because $v=\mu \circ \phi=$ $\mu \circ \psi$ and $\mu$ is faithful. Using either of these $B$-module maps, one can equip $\hat{A}$ with the structure of a $*$-algebra. We shall choose an approach that fits well with the duality on the operator-algebraic level in the next section.

First, we define an abstract Fourier transform

$$
A \rightarrow \hat{A}, \quad x \mapsto \hat{x}:=v(S(x)-),
$$

which is a linear bijection because $v$ is faithful. Evidently, $\hat{x}_{s}=\psi(S(x)-)$ and $\hat{x}_{r}=$ $\phi(S(x)-)$, and by Proposition 2.6.6, ${ }_{s} \hat{x}=\psi(-\theta(S(x)))$ and ${ }_{r} \hat{x}=\phi(-\theta(S(x)))$. For all $x, a \in A$, we define a right convolution

$$
a * \hat{x}:=\sum a_{(2)} r\left(\hat{x}_{S}\left(a_{(1)}\right)\right)=\sum a_{(2)} r\left(\psi\left(S(x) a_{(1)}\right)\right) \in A .
$$

Remark 2.7.1. One could also work with the transform $A \rightarrow \hat{A}, x \mapsto \check{x}:=$ $v(-S(x))$, and the left convolution defined by

$$
\check{x} * a:=\sum s\left({ }_{r} \check{x}\left(a_{(2)}\right)\right) a_{(1)}=\sum s\left(\phi\left(a_{(2)} S(x)\right)\right) a_{(1)} \in A \quad \text { for all } x, a \in A .
$$


If $\phi \circ S=\psi$, for example, if we are in the bi-measured case (see Remark 2.6.2 ii)), then

$\widetilde{S(x)} * S(a)=\sum S\left(\phi\left(S(a)_{(2)} S^{2}(x)\right)\right) S(a)_{(1)}=S\left(a_{(2)} r\left(\psi\left(S(x) a_{(1)}\right)\right)\right)=S(a * \hat{x})$ for all $a, x \in A$.

We collect a few useful formulas. First, for all $a, x \in A$,

$$
\begin{aligned}
a * \hat{x} & =\sum r\left(\psi\left(a_{(1)} \theta_{\bar{D}}(x)\right)\right) a_{(2)}, & & \text { (Proposition 2.6.6) } \\
a * \hat{x} & =\sum S^{-1}\left(r\left(\psi\left(S(x)_{(1)} a\right)\right) S(x)_{(2)}\right) & & \\
& =\sum x_{(1)} s\left(\psi\left(S\left(x_{(2)}\right) a\right)\right) & & \text { (Proposition 2.5.3) }
\end{aligned}
$$

Next, for all $a, x, y \in A, b \in B, \gamma, \gamma^{\prime}, \delta, \delta^{\prime} \in \Gamma$,

$$
\begin{aligned}
& r(b) a * \hat{x}=a * \widehat{s(b) x}, \quad \operatorname{ar}(b) * \hat{x}=a * \widehat{x s(b)}, \\
& s(b) a * \hat{x}=s(b)(a * \hat{x}), \quad \operatorname{as}(b) * \hat{x}=(a * \hat{x}) s(b), \\
&(a * \hat{x}) * \hat{y}=\sum a_{(3)} r\left(\psi\left(S(y) a_{(2)} r\left(\psi\left(S(x) a_{(1)}\right)\right)\right)\right) \\
&= \sum a_{(2)} r\left(\psi\left(S(y) x_{(1)} s\left(\psi\left(S\left(x_{(2)}\right) a_{(1)}\right)\right)\right)\right) \\
&= \sum a_{(2)} r\left(\psi\left(S\left(x_{(2)} r\left(\psi\left(S(y) x_{(1)}\right)\right)\right) a_{(1)}\right)\right)=a * \widehat{(x * \hat{y}),} \\
& A_{\gamma, \gamma^{\prime}} * \widehat{A_{\delta, \delta^{\prime}}} \subseteq \sum_{\gamma^{\prime \prime}} s\left(\psi\left(A_{\delta^{\prime}-1, \delta^{-1}} A_{\gamma, \gamma^{\prime \prime}}\right)\right) A_{\gamma^{\prime \prime}, \gamma^{\prime}} \subseteq \delta_{\gamma, \delta^{\prime}} A_{\delta, \gamma^{\prime}},
\end{aligned}
$$

where we used Lemma 2.6.4 in the last line. Finally, note that the surjectivity of $T_{2}$ (Proposition 2.3.8) and of $\psi$ imply

$$
\langle A * \hat{A}\rangle=(\psi \underset{B}{\otimes} \mathrm{id})\left(T_{2}\left(A_{r} \underset{B}{\otimes_{r}} r A\right)\right)=\langle A r(\psi(A))\rangle=\langle\operatorname{Ar}(B)\rangle=A .
$$

The $(B, \Gamma)^{\mathrm{ev}}$-algebra structure on $A$ induces the following structure on $\hat{A}$ :

\section{Proposition 2.7.2.}

i) $\hat{A}$ carries the structure of a non-degenerate $*$-algebra, where $\hat{y} \hat{x}=\widehat{x * \hat{y}}$ and $\hat{x}^{*}=\widehat{S(x)^{*}}$ for all $x, y \in A$.

ii) There exist non-degenerate $*$-homomorphisms $\hat{r}, \hat{s}: B \rightarrow M(\hat{A})$ such that

$$
\hat{r}(b) \hat{x}=\widehat{x r(b)}, \quad \hat{x} \hat{r}(b)=\widehat{x s(b)}, \quad \hat{s}(b) \hat{x}=\widehat{r(b) x}, \quad \hat{x} \hat{s}(b)=\widehat{s(b) x}
$$

for all $x \in A, b \in B$. The images of $\hat{r}$ and $\hat{s}$ commute.

iii) Let $\hat{A}^{\gamma, \gamma^{\prime}}=\widehat{\left(A_{\gamma, \gamma^{\prime}}\right)}$ for all $\gamma, \gamma^{\prime} \in \Gamma$. Then $\hat{A}=\bigoplus_{\gamma, \gamma^{\prime} \in \Gamma} \hat{A}^{\gamma, \gamma^{\prime}}$ as a vector space and

$$
\hat{A}^{\gamma, \gamma^{\prime}} \hat{A}^{\delta, \delta^{\prime}} \subseteq \delta_{\gamma^{\prime}, \delta} \hat{A}^{\gamma, \delta^{\prime}}, \quad\left(\hat{A}^{\gamma, \gamma^{\prime}}\right)^{*}=\hat{A}^{\gamma^{\prime}, \gamma}, \quad \hat{r}(B) \hat{s}(B) \hat{A}^{\gamma, \gamma^{\prime}} \subseteq \hat{A}^{\gamma, \gamma^{\prime}}
$$

for all $\gamma, \gamma^{\prime}, \delta, \delta^{\prime} \in \Gamma$. 
Furthermore, for all $\gamma, \gamma^{\prime}, \delta, \delta^{\prime} \in \Gamma, \hat{a} \in \hat{A}^{\gamma, \gamma^{\prime}}, b, b^{\prime} \in B$,

$$
\hat{r}(b) \hat{s}\left(b^{\prime}\right) \hat{a}=\hat{r}\left(\gamma^{-1}\left(b^{\prime}\right)\right) \hat{s}(\gamma(b)) \hat{a} \quad \text { and } \quad \hat{a} \hat{r}(b) \hat{s}\left(b^{\prime}\right)=\hat{a} \hat{r}\left(\gamma^{\prime-1}\left(b^{\prime}\right)\right) \hat{s}\left(\gamma^{\prime}(b)\right) \text {. }
$$

Proof. i) The multiplication is associative and turns $\hat{A}$ into a non-degenerate algebra by (2.19) and (2.21). The $*$-operation is involutive because $* \circ S$ is involutive, and anti-multiplicative because

$$
\begin{aligned}
S(y * \hat{x})^{*} & =\sum S\left(y_{(2)} r\left(\psi\left(S(x) y_{(1)}\right)\right)\right)^{*} \\
& =\sum S\left(y_{(2)}\right)^{*} s\left(\psi\left(y_{(1)}^{*} S(x)^{*}\right)\right) \\
& =\sum S(y)_{(1)}^{*} s\left(\psi\left(S\left(S(y)_{(2)}^{*}\right) S(x)^{*}\right)\right)=S(x)^{*} * \widehat{S(y)^{*} .}
\end{aligned}
$$

ii) For each $b \in B$, the formulas above define multipliers $\hat{r}(b), \hat{s}(b) \in M(\hat{A})$ because

$$
\hat{y}(\hat{r}(b) \hat{x})=(x r(b) * \hat{y}) \widehat{=}=(x * \widehat{y s(b)}) \widehat{=}=(\hat{y} \hat{r}(b)) \hat{x}
$$

and similarly $\hat{y}(\hat{s}(b) \hat{x})=(\hat{y} \hat{s}(b)) \hat{x}$ for all $x, y \in A$ by (2.18). The maps $\hat{r}, \hat{s}: B \rightarrow$ $M(\hat{A})$ are non-degenerate homomorphisms because $r, s: B \rightarrow M(A)$ have the same properties, their images evidently commute, and they are involutive because

$$
(\hat{x} \hat{r}(b))^{*}=(\widehat{x s(b)})^{*}=\left(S(x s(b))^{*} \widehat{)}=\left(S(x)^{*} r\left(b^{*}\right) \widehat{)}=\hat{r}\left(b^{*}\right) \hat{x}^{*}\right.\right.
$$

and similarly $(\hat{x} \hat{s}(b))^{*}=\hat{s}\left(b^{*}\right) \hat{x}^{*}$ for all $x \in A, b \in B$.

iii) All of these assertions follow easily from the definitions and relation (2.20), for example, $\hat{r}(b) \hat{x}=\widehat{x r(b)}=\left(r(\gamma(b)) x \hat{)}=\hat{s}(\gamma(b)) \hat{x}\right.$ for all $\gamma, \gamma^{\prime} \in \Gamma$, $x \in A_{\gamma, \gamma^{\prime}}, b \in B$.

\section{Construction of associated measured quantum groupoids}

In this section, we fix a measured multiplier $(B, \Gamma)$-Hopf $*$-algebroid $(A, \Delta, \epsilon, S, \mu$, $\phi, \psi)$ and construct operator-algebraic completions of this algebraic object in the form of a Hopf $C^{*}$-bimodule, Hopf-von Neumann bimodule and a measured quantum groupoid. Along the way, we shall impose further assumptions on $B, \Gamma, \mu, A$ which were mentioned already in the introduction, most notably properness.

The basic idea is to use the GNS-representations for the weight $\mu$ on the basis $B$ and the functional $v$ on the total algebra $A$, respectively. Naturally, some restrictions have to be made on $B, \Gamma, \mu$. To show that $v$ admits a bounded GNS-representation and to lift the comultiplication to the level of operator algebras, we use a fundamental unitary. To take full advantage of this unitary, we describe its domain and range as relative tensor products, and show that it is a pseudo-multiplicative unitary in the 
sense of [24] and [27]. The necessary modules are introduced in $\$ 3.2$, and the unitary itself is constructed in §3.3. This part uses Connes' spatial theory [20], and the relative tensor product of Hilbert spaces over $C^{*}$-algebras which was introduced in [22]. The fundamental unitary then gives rise to completions of $A$ and $\hat{A}$ in the form of Hopf $C^{*}$-bimodules and two Hopf-von Neumann bimodules; see \$3.4-\$3.6.To obtain the full structure of a measured quantum groupoid, we finally extend the integrals $\phi, \psi$ to the level of von Neumann algebras and show that these extensions are left- or right-invariant again in $\$ 3.7$.

Before we turn to details, let us briefly sketch the construction of the fundamental unitary, which we denote by $W$. Its domain and range can be described as separated completions of the relative tensor products ${ }_{s} A \underset{B}{\otimes}{ }_{r} A$ and ${ }_{r} A \underset{B}{\otimes} A_{r}$ with respect to the sesquilinear forms given by

$$
\begin{aligned}
\left\langle x \underset{B}{\otimes} y \mid x^{\prime} \underset{B}{\otimes} y^{\prime}\right\rangle_{\left(s, \underset{B}{\otimes_{r}} A\right)} & =v\left(x^{*} s\left(\partial_{y}\left(\phi\left(y^{*} y^{\prime}\right)\right)\right) x^{\prime}\right), \\
\langle x \underset{B}{\otimes} y)\left|x^{\prime} \underset{B}{\otimes} y^{\prime}\right\rangle_{\left(r A{ }_{B} A_{r}\right)} & =v\left(x^{*} r\left(\phi\left(y^{*} y^{\prime}\right)\right) x^{\prime}\right)
\end{aligned}
$$

for all $x, y \in A$, where $y$ is assumed to be homogeneous in the upper line. Note that positivity of these forms is not evident because $\phi$ is not assumed to be completely positive in any sense. Given that positivity, the map

$$
T_{4}: r A \underset{B}{\otimes} A_{r} \rightarrow{ }_{s} A \underset{B}{\otimes} r A, \quad x \underset{B}{\otimes} y \mapsto \Delta(y)(x \underset{B}{\otimes} 1)=\sum y_{(1)} x \underset{B}{\otimes} y_{(2)},
$$

extends to a unitary on the respective completions because it is surjective by Proposition 2.3.8 and isometric as shown by the calculation

$$
\begin{aligned}
\sum\left\langle y_{(1)} x \underset{B}{\otimes} y_{(2)} \mid y_{(1)}^{\prime} x^{\prime}{\underset{B}{\otimes}}_{(2)}^{\prime}\right\rangle_{\left(s A \otimes_{B} r A\right)} & =\sum v\left(x^{*} y_{(1)}^{*} s\left(\partial_{y_{(2)}}\left(\phi\left(y_{(2)}^{*} y_{(2)}^{\prime}\right)\right)\right) y_{(1)}^{\prime} x^{\prime}\right) \\
& =\sum v\left(x^{*} s\left(\phi\left(y_{(2)}^{*} y_{(2)}^{\prime}\right)\right) y_{(1)}^{*} y_{(1)}^{\prime} x^{\prime}\right) \\
& =v\left(x^{*} r\left(\phi\left(y^{*} y^{\prime}\right)\right) x^{\prime}\right) \\
& =\left\langle x \underset{B}{\otimes} y \mid x^{\prime} \underset{B}{\otimes} y^{\prime}\right\rangle_{\left(r A A_{B} A_{r}\right)}
\end{aligned}
$$

where $y_{(2)}$ is assumed to be homogeneous without loss of generality. The adjoint of this extension is the fundamental unitary $W$.

Similarly, one can construct and employ another unitary $V$ which is an extension of the map $T_{1}: A_{s} \underset{B}{\otimes}{ }_{s} A \rightarrow{ }_{s} A \underset{B}{\otimes} r A, x \underset{B}{\otimes} y \mapsto \Delta(x)(1 \underset{B}{\otimes} y)$. We shall focus on $W$ because this unitary is given preference in the theory of locally compact quantum groups and measured quantum groupoids.

3.1. Preparations concerning the base. We define an inner product on $B$ by $\left\langle b \mid b^{\prime}\right\rangle:=\mu\left(b^{*} b^{\prime}\right)$ for all $b, b^{\prime} \in B$, and denote by $K$ the Hilbert space obtained by completion, and by $\Lambda_{\mu}: B \rightarrow K$ the canonical inclusion. 
From now on, we assume:

(A1) The weight $\mu$ admits a GNS-representation via bounded operators in the sense that the following equivalent conditions hold:

i) for each $b \in B$, there is a $\lambda>0$ such that $\mu\left(c^{*} b^{*} b c\right) \leq \lambda \mu\left(c^{*} c\right)$ for all $c \in B$;

ii) the formula $\pi_{\mu}(b) \Lambda_{\mu}(c)=\Lambda_{\mu}(b c)$ defines a $*$-homomorphism $\pi_{\mu}: B \rightarrow \mathcal{L}(K)$.

\section{Remarks 3.1.1.}

i) Assumption (A1) holds if $B$ is a pre- $C^{*}$-algebra since then $\mu\left(c^{*} b^{*} b c\right) \leq$ $\mu\left(c^{*}\left\|b^{*} b\right\| c\right)=\left\|b^{*} b\right\| \mu\left(c^{*} c\right)$ for all $b, c \in B$. Conversely, if (A1) holds, then $B$ can be regarded as a pre- $C^{*}$-algebra with respect to the norm given by $b \mapsto\left\|\pi_{\mu}(b)\right\|$.

ii) To apply the constructions below, it may be useful to first perform a base change, similarly as described in $[21, \S 2]$, to replace $B$ by a pre- $C^{*}$-algebra of the form $C_{c}(\Omega)$, where $\Omega$ is a locally compact space with an action of $\Gamma$. For example, one can take $\Omega$ to be the set of all $*$-homomorphisms $\chi: B \rightarrow \mathbb{C}$, equipped with the weakest topology that makes the function $\Omega \rightarrow \mathbb{C}, \chi \mapsto \chi(b)$, continuous for each $b \in B$, and perform a base change along the canonical map $B \rightarrow M\left(C_{c}(\Omega)\right)$. Note, however, that such a base change can not simply be applied to left and right integrals, but only to biintegrals.

Recall that a Hilbert algebra is a $*$-algebra with an inner product such that left multiplication by each element is bounded, the resulting *-representation is nondegenerate, and the involution is pre-closed with respect to the norm induced by the inner product. Since $B$ is commutative, the map $\Lambda_{\mu}(B) \rightarrow \Lambda_{\mu}(B)$ given by $\Lambda_{\mu}(b) \mapsto \Lambda_{\mu}\left(b^{*}\right)$ extends to an anti-unitary operator $J_{\mu}$ on $K$, and hence $\Lambda_{\mu}(B) \subseteq K$ together with the *-algebra structure inherited from $B$ is a Hilbert algebra. We thus obtain

- a von Neumann algebra $N:=\pi_{\mu}(B)^{\prime \prime} \subseteq \mathcal{L}(K)$,

- a n.s.f. weight $\tilde{\mu}$ on $N$ such that $\tilde{\mu}\left(\pi_{\mu}\left(b^{*} b\right)\right)=\left\langle\Lambda_{\mu}(b) \mid \Lambda_{\mu}(b)\right\rangle=\mu\left(b^{*} b\right)$ for all $b \in B$,

- a left ideal $\mathfrak{N}_{\tilde{\mu}}:=\left\{x \in N: \tilde{\mu}\left(x^{*} x\right)<\infty\right\} \subseteq N$ of square-integrable elements,

- a closed map $\Lambda_{\tilde{\mu}}: \mathfrak{N}_{\tilde{\mu}} \rightarrow K$ such that $\left(K, \Lambda_{\tilde{\mu}}, \mathrm{id}_{N}\right)$ is a GNS-representation for $\tilde{\mu}$; this is the closure of the map $\pi_{\mu}(B) \rightarrow K$ given by $\pi_{\mu}(b) \rightarrow \Lambda_{\mu}(b)$. 
3.2. Various module structures. We define an inner product on $A$ by $\left\langle a \mid a^{\prime}\right\rangle:=$ $v\left(a^{*} a^{\prime}\right)$ for all $a, a^{\prime} \in A$ and denote by $H$ the Hilbert space obtained by completion. We call the canonical inclusion of $A$ into $H$ the GNS-map for $v$ and denote it by $\Lambda_{\nu}$.

Lemma 3.2.1. There exist maps $\Lambda_{\phi}, \Lambda_{\psi}, \Lambda_{\phi}^{\dagger}, \Lambda_{\psi}^{\dagger}: A \rightarrow \mathcal{L}(K, H)$ such that for all $x, y \in A, b \in B$,

$$
\begin{gathered}
\Lambda_{\phi}(x) \Lambda_{\mu}(b)=\Lambda_{v}(x r(b)), \\
\Lambda_{\phi}(x)^{*} \Lambda_{v}(y)=\Lambda_{\mu}\left(\phi\left(x^{*} y\right)\right), \quad \Lambda_{\phi}(x)^{*} \Lambda_{\phi}(y)=\pi_{\mu}\left(\phi\left(x^{*} y\right)\right), \\
\Lambda_{\psi}(x) \Lambda_{\mu}(b)=\Lambda_{v}(x s(b)), \\
\Lambda_{\psi}(x)^{*} \Lambda_{v}(y)=\Lambda_{\mu}\left(\psi\left(x^{*} y\right)\right), \quad \Lambda_{\psi}(x)^{*} \Lambda_{\psi}(y)=\pi_{\mu}\left(\psi\left(x^{*} y\right)\right), \\
\Lambda_{\phi}^{\dagger}(x) \Lambda_{\mu}(b)=\Lambda_{v}(r(b) x), \\
\Lambda_{\phi}^{\dagger}(x)^{*} \Lambda_{v}(y)=\Lambda_{\mu}\left(\phi\left(y \theta\left(x^{*}\right)\right)\right), \quad \Lambda_{\phi}^{\dagger}(x)^{*} \Lambda_{\phi}^{\dagger}(y)=\pi_{\mu}\left(\phi\left(y \theta\left(x^{*}\right)\right)\right), \\
\Lambda_{\psi}^{\dagger}(x) \Lambda_{\mu}(b)=\Lambda_{v}(s(b) x), \\
\Lambda_{\psi}^{\dagger}(x)^{*} \Lambda_{v}(y)=\Lambda_{\mu}\left(\psi\left(y \theta\left(x^{*}\right)\right)\right), \quad \Lambda_{\psi}^{\dagger}(x)^{*} \Lambda_{\psi}^{\dagger}(y)=\pi_{\mu}\left(\psi\left(y \theta\left(x^{*}\right)\right)\right) .
\end{gathered}
$$

Proof. We only prove the assertions concerning $\Lambda_{\phi}$ and $\Lambda_{\phi}^{\dagger}$. They follow from the relations

$$
\begin{aligned}
& \left\|\Lambda_{v}(x r(b))\right\|^{2}=v\left(r(b)^{*} x^{*} x r(b)\right)=\mu\left(b^{*} \phi\left(x^{*} x\right) b\right) \leq\left\|\pi_{\mu}\left(\phi\left(x^{*} x\right)\right)\right\|\left\|\Lambda_{\mu}(b)\right\|^{2} \\
& \left\langle\Lambda_{v}(y) \mid \Lambda_{v}(x r(b))\right\rangle=v\left(y^{*} x r(b)\right)=\mu\left(\phi\left(y^{*} x\right) b\right)=\left\langle\Lambda_{\mu}\left(\phi\left(x^{*} y\right)\right) \mid \Lambda_{\mu}(b)\right\rangle
\end{aligned}
$$

and

$$
\begin{aligned}
\left\|\Lambda_{v}(r(b) x)\right\|^{2}=v\left(x^{*} r\left(b^{*} b\right) x\right) & =v\left(\theta^{-1}(x) x^{*} r\left(b^{*} b\right)\right) \\
& =\mu\left(\phi\left(\theta^{-1}(x) x^{*}\right) b^{*} b\right) \\
& \leq\left\|\Lambda_{\mu}(b)\right\|^{2}\left\|\pi_{\mu}\left(\theta^{-1}(x) x^{*}\right)\right\|, \\
\left\langle\Lambda_{v}(y) \mid \Lambda_{v}(r(b) x)\right\rangle=v\left(y^{*} r(b) x\right) & =v\left(\theta^{-1}(x) y^{*} r(b)\right) \\
& =\mu\left(\phi\left(\theta^{-1}(x) y^{*}\right) b\right) \\
& =\left\langle\Lambda_{\mu}\left(\phi\left(y \theta\left(x^{*}\right)\right)\right) \mid \Lambda_{\mu}(b)\right\rangle,
\end{aligned}
$$

which hold for all $x, y \in A$ and $b \in B$.

The maps introduced above yield various module structures on $H$ as follows. Let

$$
E_{\phi}:=\left[\Lambda_{\phi}(A)\right], \quad E_{\psi}:=\left[\Lambda_{\psi}(A)\right], \quad E_{\phi}^{\dagger}:=\left[\Lambda_{\phi}^{\dagger}(A)\right], \quad E_{\psi}^{\dagger}:=\left[\Lambda_{\psi}^{\dagger}(A)\right] .
$$

We shall use the following concepts introduced in [22, 24]. A $C^{*}-\mathfrak{b}$-module, where $\mathfrak{b}=\left(K,\left[\pi_{\mu}(B)\right],\left[\pi_{\mu}(B)\right]\right)$, consists of a Hilbert space $L$ and a closed subset $E \subseteq \mathcal{L}(K, L)$ such that $[E K]=L,\left[E \pi_{\mu}(B)\right]=E,\left[E^{*} E\right]=\left[\pi_{\mu}(B)\right]$. Each 
such $C^{*}$-b-module gives rise to a normal, faithful, non-degenerate representation $\rho_{E}: N=\pi_{\mu}(B)^{\prime \prime} \rightarrow \mathcal{L}(L)$ such that $\rho_{E}(x) \xi=\xi x$ for all $x \in N, \xi \in E$. A $C^{*}-$ $(\mathfrak{b}, \mathfrak{b})$-module is a triple $(L, E, F)$ such that $(L, E)$ and $(L, F)$ are $C^{*}$-b-modules and $\left[\rho_{E}\left(\pi_{\mu}(B)\right) F\right]=F$ and $\left[\rho_{F}\left(\pi_{\mu}(B)\right) E\right]=[E]$.

Lemma 3.2.2. The Hilbert space $H$ is a $C^{*}-(\mathfrak{b}, \mathfrak{b})$-module with respect to either two of the spaces $E_{\phi}, E_{\psi}, E_{\phi}^{\dagger}, E_{\psi}^{\dagger}$. The representations $\alpha:=\rho_{E_{\phi}^{\dagger}}, \beta:=\rho_{E_{\psi}^{\dagger}}$, $\widehat{\alpha}:=\rho_{E_{\psi}}, \widehat{\beta}:=\rho_{E_{\phi}}$ of $N$ on $H$ are given by

$$
\begin{array}{ll}
\alpha\left(\pi_{\mu}(b)\right) \Lambda_{v}(a)=\Lambda_{v}(r(b) a), & \beta\left(\pi_{\mu}(b)\right) \Lambda_{v}(a)=\Lambda_{v}(s(b) a), \\
\widehat{\beta}\left(\pi_{\mu}(b)\right) \Lambda_{v}(a)=\Lambda_{v}(\operatorname{ar}(b)), & \widehat{\alpha}\left(\pi_{\mu}(b)\right) \Lambda_{v}(a)=\Lambda_{v}(a s(b))
\end{array}
$$

for all $b \in B, a \in A$.

Proof. Let $E, F$ be any two of the spaces listed above. Then $[E H]=H$ and $\left[E \pi_{\mu}(B)\right]=E$ because $\langle r(B) s(B) \operatorname{Ar}(B) s(B)\rangle=A$, and $\left[E^{*} E\right]=\left[\pi_{\mu}(B)\right]$ because $\phi(A)=B=\psi(A)$. Thus, $(H, E)$ is a $C^{*}$-b-module. The formulas for the associated representations are easily verified. Using these formulas and the relation $\langle r(B) s(B) \operatorname{Ar}(B) s(B)\rangle=A$, one easily checks that $\left[\rho_{E}\left(\pi_{\mu}(B)\right) F\right]=F$ and $\left[\rho_{F}\left(\pi_{\mu}(B)\right) E\right]=E$.

Recall that a vector $\zeta$ in a Hilbert space $L$ is bounded with respect to a normal, non-degenerate representation $\rho: N \rightarrow \mathcal{L}(L)$ and the weight $\tilde{\mu}$ if the following equivalent conditions hold:

i) there exists a $K \geq 0$ such that $\|\rho(x) \zeta\| \leq K \tilde{\mu}\left(x^{*} x\right)$ for all $x \in \mathfrak{N}_{\tilde{\mu}}$;

ii) there exists an operator $R_{\zeta}^{\rho, \tilde{\mu}} \in \mathcal{L}(K, L)$ such that $R_{\zeta}^{\rho, \tilde{\mu}} \Lambda_{\mu}(x)=\rho(x) \zeta$ for all $x \in \mathfrak{N}_{\tilde{\mu}}$.

The set of all such bounded vectors is denoted by $D\left(L_{\rho}, \tilde{\mu}\right)$. This spaces carries an $N$-valued inner product $\langle-\mid-\rangle_{\rho, \tilde{\mu}}$, given by $\left\langle\zeta \mid \zeta^{\prime}\right\rangle_{\rho, \tilde{\mu}}=\left(R_{\zeta}^{\rho, \tilde{\mu}}\right)^{*} R_{\zeta^{\prime}}^{\rho, \tilde{\mu}}$ for all $\zeta, \zeta^{\prime} \in D\left(L_{\rho}, \tilde{\mu}\right)$, and $\rho(N)^{\prime} D\left(L_{\rho}, \tilde{\mu}\right)=D\left(L_{\rho}, \tilde{\mu}\right)$ and

$\Lambda_{\tilde{\mu}}\left(\left\langle\zeta \mid \zeta^{\prime}\right\rangle_{\rho, \tilde{\mu}}\right)=\left(R_{\zeta}^{\rho, \tilde{\mu}}\right)^{*} \zeta^{\prime}, \quad R_{T \zeta}^{\rho, \tilde{\mu}}=T R_{\zeta}^{\rho, \tilde{\mu}} \quad$ for all $T \in \rho(N)^{\prime}, \zeta, \zeta^{\prime} \in D\left(L_{\rho}, \tilde{\mu}\right)$.

Lemma 3.2.3. $\Lambda_{v}(A) \subseteq D\left(H_{\alpha}, \tilde{\mu}\right) \cap D\left(H_{\beta}, \tilde{\mu}\right) \cap D\left(H_{\widehat{\alpha}}, \tilde{\mu}\right) \cap D\left(H_{\widehat{\beta}}, \tilde{\mu}\right)$ and for all $x, y \in A$,

$$
R_{\Lambda_{v}(x)}^{\alpha, \tilde{\mu}}=\Lambda_{\phi}^{\dagger}(x), \quad R_{\Lambda_{v}(x)}^{\beta, \tilde{\mu}}=\Lambda_{\psi}^{\dagger}(x), \quad R_{\Lambda_{v}(x)}^{\widehat{\alpha}, \tilde{\mu}}=\Lambda_{\psi}(x), \quad R_{\Lambda_{v}(x)}^{\widehat{\beta}, \tilde{\mu}}=\Lambda_{\phi}(x) .
$$

Proof. We shall only prove the assertion concerning $\alpha$. Let $a \in A$. Then $\Lambda_{\phi}^{\dagger}(a) \Lambda_{\tilde{\mu}}\left(\pi_{\mu}(b)\right)=\Lambda_{\nu}(r(b) a)=\alpha\left(\pi_{\mu}(b)\right) \Lambda_{\nu}(a)$ for all $b \in B$, and since $\pi_{\mu}(B)$ is a core for $\Lambda_{\tilde{\mu}}$, we can conclude $\Lambda_{\phi}^{\dagger}(a) \Lambda_{\tilde{\mu}}(x)=\alpha(x) \Lambda_{v}(a)$ for all $x \in \mathfrak{N}_{\tilde{\mu}}$. 
The preceding result and Lemma 3.2.1 imply that for all $x, y \in A$,

$$
\begin{array}{lll}
\left\langle\Lambda_{v}(x) \mid \Lambda_{v}(y)\right\rangle_{\alpha, \tilde{\mu}}=\pi_{\mu}\left(\phi\left(y \theta\left(x^{*}\right)\right)\right), & \left\langle\Lambda_{v}(x) \mid \Lambda_{v}(y)\right\rangle_{\beta, \tilde{\mu}}=\pi_{\mu}\left(\psi\left(y \theta\left(x^{*}\right)\right)\right), \\
\left\langle\Lambda_{v}(x) \mid \Lambda_{v}(y)\right\rangle_{\hat{\alpha}, \tilde{\mu}}=\pi_{\mu}\left(\psi\left(x^{*} y\right)\right), & & \left\langle\Lambda_{v}(x) \mid \Lambda_{v}(y)\right\rangle_{\hat{\beta}, \tilde{\mu}}=\pi_{\mu}\left(\phi\left(x^{*} y\right)\right) .
\end{array}
$$

3.3. The fundamental unitary. To define the domain and the range of the fundamental unitary, we use Connes' relative tensor product of Hilbert modules and the module structures introduced above. Connes' original manuscript on the construction remained unpublished; we therefore refer to [20] and [23] for details.

The relative tensor product $H_{\beta} \underset{\widetilde{\mu}}{\otimes_{\alpha} H}$ is the separated completion of the algebraic tensor product $D\left(H_{\beta}, \tilde{\mu}\right) \otimes K \otimes D\left(H_{\alpha}, \tilde{\mu}\right)$ with respect to the sesquilinear form given by

$$
\left\langle\xi \otimes \zeta \otimes \eta \mid \xi^{\prime} \otimes \zeta^{\prime} \otimes \eta^{\prime}\right\rangle=\left\langle\zeta \mid\left\langle\xi \mid \xi^{\prime}\right\rangle_{\beta, \tilde{\mu}}\left\langle\eta \mid \eta^{\prime}\right\rangle_{\alpha, \tilde{\mu}} \zeta^{\prime}\right\rangle .
$$

This Hilbert space can naturally be identified with the separated completions of the algebraic tensor products $D\left(H_{\beta}, \tilde{\mu}\right) \otimes H$ and $H \otimes D\left(H_{\alpha}, \tilde{\mu}\right)$ with respect to the sesquilinear forms given by

$\left\langle\xi \otimes \eta \mid \xi^{\prime} \otimes \eta^{\prime}\right\rangle=\left\langle\eta \mid \alpha\left(\left\langle\xi \mid \xi^{\prime}\right\rangle_{\beta, \tilde{\mu}}\right) \eta^{\prime}\right\rangle \quad$ and $\quad\left\langle\xi \otimes \eta \mid \xi^{\prime} \otimes \eta^{\prime}\right\rangle=\left\langle\xi \mid \beta\left(\left\langle\eta \mid \eta^{\prime}\right\rangle_{\alpha, \tilde{\mu}}\right) \xi^{\prime}\right\rangle$,

respectively, via

$$
\xi \otimes R_{\xi}^{\alpha, \tilde{\mu}} \zeta \equiv \xi \otimes \zeta \otimes \eta \equiv R_{\xi}^{\beta, \tilde{\mu}} \zeta \otimes \eta,
$$

and we shall use these identifications without further notice. Replacing the representations $\beta, \alpha$ by $\alpha, \widehat{\beta}$ or $\widehat{\alpha}, \beta$, respectively, one obtains the relative tensor products $H_{\alpha} \underset{\widetilde{\mu}}{\otimes_{\widehat{\beta}}} H$ and $H_{\widehat{\alpha}} \otimes_{\widetilde{\mu}} \beta H$.

To proceed, we impose the following simplifying assumption:

(A2) The Radon-Nikodym cocycle $\left(D_{\gamma}\right)_{\gamma}$ of $\mu$ has a positive square root in $M(B)$ in the sense that there exists a family $\left(D_{\gamma}^{\frac{1}{2}}\right)_{\gamma \in \Gamma}$ in $M(B)$ such that for all $\gamma, \gamma^{\prime} \in \Gamma, c \in B$,

$$
\begin{gathered}
D_{e}^{\frac{1}{2}}=1, \quad\left(D_{\gamma}^{\frac{1}{2}}\right)^{*}=D_{\gamma}^{\frac{1}{2}}, \quad\left(D_{\gamma}^{\frac{1}{2}}\right)^{2}=D_{\gamma}, \\
D_{\gamma \gamma^{\prime}}^{\frac{1}{2}}=\gamma^{\prime-1}\left(D_{\gamma}^{\frac{1}{2}}\right) D_{\gamma^{\prime}}^{\frac{1}{2}}, \quad \mu\left(c^{*} D_{\gamma}^{\frac{1}{2}} c\right) \geq 0 .
\end{gathered}
$$

Clearly, this condition implies the existence of a unitary representation $U: \Gamma \rightarrow$ $\mathcal{L}(K)$ such that

$$
U_{\gamma} \Lambda_{\mu}(c)=\Lambda_{\mu}\left(\gamma\left(c D_{\gamma}^{\frac{1}{2}}\right)\right), \quad U_{\gamma} \pi_{\mu}(b) U_{\gamma}^{*}=\pi_{\mu}(\gamma(b)) \quad \text { for all } b, c \in B, \gamma \in \Gamma \text {. }
$$


Similarly as in (2.9), we define linear maps $D^{\frac{1}{2}}, \bar{D}^{\frac{1}{2}}: A \rightarrow A$ by

$$
D^{\frac{1}{2}}(a)=r\left(D_{\partial_{a}^{-1}}^{\frac{1}{2}}\right) a=\operatorname{ar}\left(D_{\partial_{a}}^{-\frac{1}{2}}\right), \quad \bar{D}^{\frac{1}{2}}(a)=s\left(D_{\bar{\partial}_{a}^{-1}}^{\frac{1}{2}}\right) a=\operatorname{as}\left(D_{\bar{\partial}_{a}}^{-\frac{1}{2}}\right)
$$

for all homogeneous $a \in A$. These maps share all the properties of the maps $D, \bar{D}$ listed in Lemma 2.6.3. Short calculations show that for all homogeneous $x, y \in A$,

$$
\begin{aligned}
& \Lambda_{\phi}(x) U_{\partial_{x}^{-1}}=\Lambda_{\phi}^{\dagger}\left(D^{\frac{1}{2}}(x)\right), \quad\left\langle\Lambda_{\nu}\left(D^{\frac{1}{2}}(x)\right) \mid \Lambda_{\nu}\left(D^{\frac{1}{2}}(y)\right)\right\rangle_{\alpha, \tilde{\mu}}=\pi_{\mu}\left(\partial_{x}\left(\phi\left(x^{*} y\right)\right)\right), \\
& \Lambda_{\psi}(x) U_{\bar{\partial}_{\bar{x}}^{-1}}=\Lambda_{\psi}^{\dagger}\left(\bar{D}^{\frac{1}{2}}(x)\right), \quad\left\langle\Lambda_{\nu}\left(\bar{D}^{\frac{1}{2}}(x)\right) \mid \Lambda_{\nu}\left(\bar{D}^{\frac{1}{2}}(y)\right)\right\rangle_{\beta, \tilde{\mu}}=\pi_{\mu}\left(\bar{\partial}_{x}\left(\psi\left(x^{*} y\right)\right)\right) .
\end{aligned}
$$

Indeed, for all homogeneous $x, y \in A$ and $b \in B$,

$$
\begin{aligned}
\Lambda_{\phi}(x) U_{\partial_{x}^{-1}} \Lambda_{\mu}(b) & =\Lambda_{v}\left(x r\left(\partial_{x}^{-1}\left(b D_{\partial_{x}^{-1}}^{\frac{1}{2}}\right)\right)\right. \\
& =\Lambda_{v}\left(r\left(b D_{\partial_{x}^{-1}}^{\frac{1}{2}}\right) x\right)=\Lambda_{\phi}^{\dagger}\left(D^{\frac{1}{2}}(x)\right) \Lambda_{\mu}(b), \\
\Lambda_{\phi}^{\dagger}\left(D^{\frac{1}{2}}(x)\right)^{*} \Lambda_{\phi}^{\dagger}\left(D^{\frac{1}{2}}(y)\right) & =U_{\partial_{x}^{-1}}^{*} \Lambda_{\phi}(x)^{*} \Lambda_{\phi}(y) U_{\partial_{y}^{-1}} \\
& =U_{\partial_{x}} \pi_{\mu}\left(\phi\left(x^{*} y\right)\right) U_{\partial_{y}^{-1}}=\pi_{\mu}\left(\partial_{x}\left(\phi\left(x^{*} y\right)\right)\right) .
\end{aligned}
$$

Lemma 3.3.1. The sesquilinear forms on ${ }_{s} A \underset{B}{\otimes} \otimes_{r} A$ and ${ }_{r} A \underset{B}{\otimes} A_{r}$ defined in (3.1) are positive. Denote by $\overline{{ }_{s} A \otimes_{B} \otimes_{r} A}$ and $\overline{{ }_{r} A \otimes_{B} A_{r}}$ the respective separated completions. Then there exist isomorphisms

$$
\begin{aligned}
& \Lambda: \overline{{ }_{r} A \underset{B}{\otimes} A_{r}} \rightarrow H_{\alpha} \underset{\widetilde{\mu}}{\bigotimes_{\beta}} H, \quad x \underset{B}{\otimes} y \mapsto \Lambda_{v}(x) \underset{\widetilde{\mu}}{\otimes_{B}} \Lambda_{v}(y),
\end{aligned}
$$

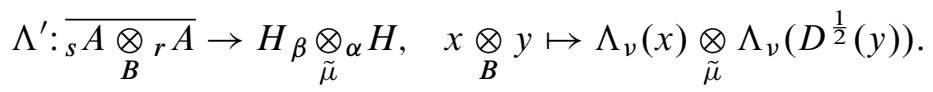

Proof. The maps $\Lambda, \Lambda^{\prime}$ are surjective because $\Lambda_{v}(A) \subseteq H$ is dense, and they are well-defined and isometric because (3.8), (3.6) and (3.11) imply for all homogeneous $x, y \in A$

$$
\begin{aligned}
\left\langle\Lambda(x \otimes y) \mid \Lambda\left(x^{\prime} \otimes y^{\prime}\right)\right\rangle & =v\left(x^{*} s\left(\phi\left(y^{*} y^{\prime}\right)\right) x^{\prime}\right) \\
\left\langle\Lambda^{\prime}(x \otimes y) \mid \Lambda^{\prime}\left(x^{\prime} \otimes y^{\prime}\right)\right\rangle & =v\left(x^{*} r\left(\partial_{y}\left(\phi\left(y^{*} y^{\prime}\right)\right)\right) x^{\prime}\right) .
\end{aligned}
$$




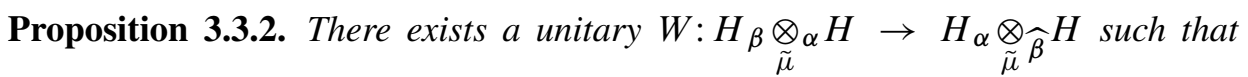
$W^{*} \circ \Lambda=\Lambda^{\prime} \circ T_{4}$ as maps from ${ }_{r} A \underset{B}{\otimes} A_{r}$ to $H_{\beta} \underset{\tilde{\mu}}{\otimes_{\alpha}} H$, that is, for all homogeneous $x, y \in A$,

$$
\begin{aligned}
W^{*}\left(\Lambda_{v}(x) \underset{\widetilde{\mu}}{\otimes} \Lambda_{v}(y)\right) & =\sum \Lambda_{v}\left(\bar{D}^{\frac{1}{2}}\left(y_{(1)}\right) x\right) \underset{\widetilde{\mu}}{\otimes} \Lambda_{v}\left(y_{(2)}\right) \\
& =\sum \Lambda_{v}\left(y_{(1)} x\right) \underset{\widetilde{\mu}}{\otimes} \Lambda_{v}\left(D^{\frac{1}{2}}\left(y_{(2)}\right)\right) \\
W\left(\Lambda_{v}(x) \underset{\widetilde{\mu}}{\otimes} \Lambda_{v}(y)\right) & =\sum \Lambda_{v}\left(S^{-1}\left(D^{-\frac{1}{2}}\left(y_{(1)}\right)\right) x\right) \underset{\widetilde{\mu}}{\otimes} \Lambda_{v}\left(y_{(2)}\right) \\
& =\sum \Lambda_{v}\left(\bar{D}^{\frac{1}{2}}\left(S^{-1}\left(y_{(1)}\right)\right) x\right) \underset{\widetilde{\mu}}{\otimes} \Lambda_{v}\left(y_{(2)}\right) .
\end{aligned}
$$

Proof. Calculation (3.2) and Lemma 3.3.1 imply that the map $\Lambda_{v}(x) \underset{\widetilde{\mu}}{\otimes} \Lambda_{v}(y) \mapsto$ $\sum \Lambda_{v}\left(y_{(1)} x\right) \underset{\widetilde{\mu}}{\otimes} \Lambda_{v}\left(D^{\frac{1}{2}}\left(y_{(2)}\right)\right)$ extends to an isometry $H_{\alpha} \underset{\widetilde{\mu}}{\bigotimes_{\widehat{\beta}}} H \rightarrow H_{\beta} \underset{\widetilde{\mu}}{\otimes_{\alpha}} H$. Bijectivity of this isometry and the formula for $W$ follow from Proposition 2.3.8 and Lemma 2.6.3.

Similarly, the map $T_{1}$ yields a second fundamental unitary:

Proposition 3.3.3. There exists a unitary $V: H_{\widehat{\alpha}} \underset{\tilde{\mu}}{\bigotimes_{\beta}} H \rightarrow H_{\beta} \underset{\widetilde{\mu}}{\bigotimes_{\alpha} H}$ such that for all homogeneous $x, y \in A$,

$$
\begin{aligned}
V\left(\Lambda_{v}(x) \underset{\widetilde{\mu}}{\otimes} \Lambda_{v}(y)\right) & =\sum \Lambda_{v}\left(\bar{D}^{\frac{1}{2}}\left(x_{(1)}\right)\right) \underset{\widetilde{\mu}}{\otimes} \Lambda_{v}\left(x_{(2)} y\right) \\
& =\sum \Lambda_{v}\left(x_{(1)}\right) \underset{\widetilde{\mu}}{\otimes} \Lambda_{v}\left(D^{\frac{1}{2}}\left(x_{(2)}\right) y\right), \\
V^{*}\left(\Lambda_{v}(x) \underset{\widetilde{\mu}}{\otimes} \Lambda_{v}(y)\right) & =\sum \Lambda_{v}\left(x_{(1)}\right) \underset{\widetilde{\mu}}{\otimes} \Lambda_{v}\left(S\left(\bar{D}^{-\frac{1}{2}}\left(x_{(2)}\right)\right) y\right) \\
& =\sum \Lambda_{v}\left(x_{(1)}\right) \underset{\widetilde{\mu}}{\otimes} \Lambda_{v}\left(D^{\frac{1}{2}}\left(S\left(x_{(2)}\right)\right) y\right) .
\end{aligned}
$$

Proof. The formula above defines an isometry $V$. Indeed, (3.8), (3.6) and (3.12) imply

$$
\begin{aligned}
& \sum\left\langle\Lambda_{v}\left(\bar{D}^{\frac{1}{2}}\left(x_{(1)}\right)\right) \underset{\widetilde{\mu}}{\otimes} \Lambda_{v}\left(x_{(2)} y\right) \mid \Lambda_{v}\left(\bar{D}^{\frac{1}{2}}\left(x_{(1)}^{\prime}\right)\right) \underset{\widetilde{\mu}}{\underset{\sim}{\otimes}} \Lambda_{v}\left(x_{(2)}^{\prime} y^{\prime}\right)\right\rangle_{\left(H_{\beta} \underset{\tilde{\mu}}{\left.\otimes_{\alpha} H\right)}\right.} \\
& =\sum v\left(y^{*} x_{(2)}^{*} r\left(\bar{\partial}_{x_{(1)}}\left(\psi\left(x_{(1)}^{*} x_{(1)}^{\prime}\right)\right)\right) x_{(2)}^{\prime} y^{\prime}\right) \\
& =\sum v\left(y^{*} x_{(2)}^{*} x_{(2)}^{\prime} r\left(\psi\left(x_{(1)}^{*} x_{(1)}^{\prime}\right)\right) y^{\prime}\right), \\
& \left\langle\Lambda_{v}(x) \underset{\widetilde{\mu}}{\otimes} \Lambda_{v}(y) \mid \Lambda_{v}\left(x^{\prime}\right) \underset{\widetilde{\mu}}{\otimes} \Lambda_{v}\left(y^{\prime}\right)\right\rangle_{\left(H_{\alpha \sim \mu} \mathcal{\otimes}_{\beta} H\right)}=v\left(y^{*} s\left(\psi\left(x^{*} x^{\prime}\right)\right) y^{\prime}\right)
\end{aligned}
$$


for all homogeneous $x, x^{\prime}, y, y^{\prime} \in A$, where $x_{(1)}$ is assumed to be homogeneous without loss of generality, and by right-invariance of $\psi$ (see Remark 2.5.2 i)), the expressions above coincide. Bijectivity of $V$ and the inversion formula follow from Proposition 2.3.8.

3.4. Boundedness of the canonical representations. The first application of the fundamental unitary $W$ is to show that left multiplication on $A$ and right convolution by $\hat{A}$ extend to representations on the Hilbert space $H$.

The proof of Theorem 3.4.2 involves operators and slice maps of the following form. For each $\xi \in D\left(H_{\beta}, \tilde{\mu}\right)$ and $\eta \in D\left(H_{\alpha}, \tilde{\mu}\right)$, there exist bounded linear operators

$$
\lambda_{\xi}^{\beta, \alpha}: H \rightarrow H_{\beta} \underset{\tilde{\mu}}{\otimes_{\alpha}} H, \eta^{\prime} \mapsto \xi \underset{\tilde{\mu}}{\otimes} \eta^{\prime}, \quad \rho_{\eta}^{\beta, \alpha}: H \rightarrow H_{\beta} \underset{\tilde{\mu}}{\mathbb{\sim}_{\alpha} H,} \xi^{\prime} \mapsto \xi^{\prime} \underset{\tilde{\mu}}{\otimes} \eta,
$$

whose adjoints are given by

$$
\left(\lambda_{\xi}^{\beta, \alpha}\right)^{*}\left(\xi^{\prime} \otimes \eta^{\prime}\right)=\alpha\left(\left\langle\xi \mid \xi^{\prime}\right\rangle_{\beta, \tilde{\mu}}\right) \eta^{\prime}, \quad\left(\rho_{\eta}^{\beta, \alpha}\right)^{*}\left(\xi^{\prime} \otimes \eta^{\prime}\right)=\beta\left(\left\langle\eta \mid \eta^{\prime}\right\rangle_{\alpha, \tilde{\mu}}\right) \xi^{\prime}
$$

see also [4]. Likewise, there exist operators $\lambda_{\xi}^{\alpha, \widehat{\beta}}, \rho_{\eta}^{\alpha, \widehat{\beta}}: H \rightarrow H_{\alpha} \underset{\widetilde{\mu}}{\otimes} \widehat{\beta} H$ for all $\xi \in$ $D\left(H_{\alpha}, \tilde{\mu}\right)$ and $\eta \in D\left(H_{\widehat{\beta}}, \tilde{\mu}\right)$ which are defined similarly. Using these operators, one defines slice maps

$$
\begin{gathered}
\omega_{\xi, \xi^{\prime}} * \operatorname{id}: \mathcal{L}\left(H_{\alpha} \underset{\widetilde{\mu}}{\otimes_{\widehat{\beta}}} H, H_{\beta} \underset{\widetilde{\mu}}{\left.\otimes_{\alpha} H\right)} \rightarrow \mathcal{L}(H), \quad T \mapsto\left(\lambda_{\xi}^{\beta, \alpha}\right)^{*} T \lambda_{\xi^{\prime}}^{\alpha, \widehat{\beta}},\right. \\
\operatorname{id} * \omega_{\eta, \eta^{\prime}}: \mathcal{L}\left(H_{\alpha} \underset{\widetilde{\mu}}{\otimes_{\widehat{\beta}}} H, H_{\beta} \underset{\tilde{\mu}}{\left.\otimes_{\alpha} H\right)} \rightarrow \mathcal{L}(H), \quad T \mapsto\left(\rho_{\eta}^{\beta, \alpha}\right)^{*} T \rho_{\eta^{\prime}}^{\alpha, \widehat{\beta}}\right.
\end{gathered}
$$

for all $\xi \in D\left(H_{\beta}, \tilde{\mu}\right), \xi^{\prime} \in D\left(H_{\alpha}, \tilde{\mu}\right), \eta \in D\left(H_{\alpha}, \tilde{\mu}\right), \eta^{\prime} \in D\left(H_{\widehat{\beta}}, \tilde{\mu}\right)$; see [3].

Lemma 3.4.1. Let $x, x^{\prime}, y, y^{\prime} \in A$. Then

$$
\begin{gathered}
\left(\operatorname{id} * \omega_{\Lambda_{v}(y), \Lambda_{v}\left(y^{\prime}\right)}\right)\left(W^{*}\right) \Lambda_{v}(x)=\Lambda_{v}(\text { ax }), \text { where } a=\sum \bar{D}^{-\frac{1}{2}}\left(y_{(1)}^{\prime} s\left(\phi\left(y^{*} y_{(2)}^{\prime}\right)\right)\right) \\
\left(\omega_{\Lambda_{v}(x), \Lambda_{v}\left(x^{\prime}\right)} * \mathrm{id}\right)\left(W^{*}\right) \Lambda_{v}(y)=\Lambda_{v}(y * \hat{c}), \text { where } c=S^{-1}\left(\bar{D}^{\frac{1}{2}}\left(\theta^{-1}\left(x^{\prime}\right) x^{*}\right)\right)
\end{gathered}
$$


Proof. Without loss of generality, we may assume $y$ to be homogeneous. Then

$$
\begin{aligned}
\left(\rho_{\Lambda_{v}(y)}^{\beta, \alpha}\right)^{*} W^{*} & \rho_{\Lambda_{v}\left(y^{\prime}\right)}^{\alpha, \widehat{\beta}} \Lambda_{v}(x) \\
& =\sum\left(\rho_{\Lambda_{v}(y)}^{\beta, \alpha}\right)^{*}\left(\Lambda_{v}\left(y_{(1)}^{\prime} x\right) \underset{\widetilde{\mu}}{\otimes} \Lambda_{v}\left(D^{\frac{1}{2}}\left(y_{(2)}^{\prime}\right)\right)\right) \\
& =\sum \beta\left(\left\langle\Lambda_{v}(y) \mid \Lambda_{v}\left(D^{\frac{1}{2}}\left(y_{(2)}^{\prime}\right)\right)\right\rangle_{\alpha, \tilde{\mu}}\right) \Lambda_{v}\left(y_{(1)}^{\prime} x\right) \\
& =\sum \Lambda_{v}\left(s\left(\partial_{y}\left(\phi\left(D^{-\frac{1}{2}}(y)^{*} y_{(2)}^{\prime}\right)\right)\right) y_{(1)}^{\prime} x\right) \\
& =\sum \Lambda_{v}\left(y_{(1)}^{\prime} s\left(\phi\left(y^{*} D^{-\frac{1}{2}}\left(y_{(2)}^{\prime}\right)\right)\right) x\right) \\
& =\sum \Lambda_{v}\left(\bar{D}^{-\frac{1}{2}}\left(y_{(1)^{\prime}}^{\prime}\left(\phi\left(y^{*} y_{(2)}^{\prime}\right)\right)\right) x\right) \\
\left(\lambda_{\Lambda_{v}(x)}^{\beta, \alpha}\right)^{*} W^{*} & \lambda_{\Lambda_{v}\left(x^{\prime}\right)}^{\alpha} \Lambda_{v}(y) \\
& =\sum\left(\lambda_{\Lambda_{v}(x)}^{\beta, \alpha}\right)^{*}\left(\Lambda_{v}\left(\bar{D}^{\frac{1}{2}}\left(y_{(1)}\right) x^{\prime}\right) \underset{\tilde{\mu}}{\otimes} \Lambda_{v}\left(y_{(2)}\right)\right) \\
& =\sum \alpha\left(\left\langle\Lambda_{v}(x) \mid \Lambda_{v}\left(\bar{D}^{\frac{1}{2}}\left(y_{(1)}\right) x^{\prime}\right)\right\rangle_{\beta, \tilde{\mu}}\right) \Lambda_{v}\left(y_{(2)}\right)
\end{aligned}
$$

Theorem 3.4.2. Let $(A, \Delta, \epsilon, S, \mu, \phi, \psi)$ be a measured multiplier $(B, \Gamma)$-Hopf *-algebroid such that $\mu$ admits a GNS-representation via bounded operators $\left(K, \Lambda_{\mu}, \pi_{\mu}\right)$ and its Radon-Nikodym cocycle has a positive square root in $M(B)$. Denote by $\Lambda_{v}: A \rightarrow \mathcal{L}(H)$ the GNS-map for $v=\mu \circ \phi=\mu \circ \psi$. Then there exist non-degenerate $*$-homomorphisms $\pi_{\nu}: A \rightarrow \mathcal{L}(H)$ and $\rho: \hat{A} \rightarrow \mathcal{L}(H)$ such that

$$
\pi_{v}(x) \Lambda_{v}(y)=\Lambda_{v}(x y) \text { and } \rho(\omega) \Lambda_{v}(y)=\Lambda_{v}(y * \omega)
$$

for all $x, y \in A, \omega \in \hat{A}$.

Proof of Theorem 3.4.2. For elements $a$ and $c$ of the form in Lemma 3.4.1, the maps $\Lambda_{v}(y) \mapsto \Lambda_{v}(a y)$ and $\Lambda_{v}(x) \mapsto \Lambda_{v}(x * \hat{c})$ coincide with compositions of bounded operators and therefore are bounded. Since elements of this form span $A$, we obtain maps $\pi_{v}: A \rightarrow \mathcal{L}(H)$ and $\rho: \hat{A} \rightarrow \mathcal{L}(H)$ as in (3.15). Evidently, $\pi_{v}$ is a *-homomorphism. The map $\rho$ is multiplicative by (2.19) and Proposition 2.7.2, and 
it is involutive because by (2.17) and Proposition 2.7.2,

$$
\begin{aligned}
\left\langle\rho(\hat{x})^{*} \Lambda_{v}(z) \mid \Lambda_{v}(y)\right\rangle & =\left\langle\rho \left(\widehat{\left.S(x)^{*}\right) \Lambda_{v}(z)\left|\Lambda_{v}(y)\right\rangle}\right.\right. \\
& =\sum\left\langle\Lambda_{v}\left(S(x)_{(1)}^{*} s\left(\psi\left(S\left(S(x)_{(2)}^{*}\right) z\right)\right)\right) \mid \Lambda_{v}(y)\right\rangle \\
& =v\left(s\left(\psi\left(z^{*} x_{(1)}\right)\right) S\left(x_{(2)}\right) y\right) \\
& =v\left(z^{*} x_{(1)} s\left(\psi\left(S\left(x_{(2)}\right) y\right)\right)\right)=\left\langle\Lambda_{v}(z) \mid \rho(\hat{x}) \Lambda_{v}(y)\right\rangle .
\end{aligned}
$$

Finally, $\pi_{v}$ and $\rho$ are non-degenerate because $\langle A A\rangle=A$ and $\langle A * \hat{A}\rangle=A$ (see (2.21)).

Remark 3.4.3. Lemma 3.4.1, Theorem 3.4.2 and self-adjointness of $\pi_{v}(A)$ and $\rho(\hat{A})$ imply

$$
\begin{aligned}
\pi_{v}(A) & =\operatorname{span}\left\{\left(\operatorname{id} * \omega_{\Lambda_{v}(y), \Lambda_{v}\left(y^{\prime}\right)}\right)\left(W^{*}\right) \mid y, y^{\prime} \in A\right\} \\
& =\operatorname{span}\left\{\left(\operatorname{id} * \omega_{\Lambda_{v}(y), \Lambda_{v}\left(y^{\prime}\right)}\right)(W) \mid y, y^{\prime} \in A\right\}, \\
\rho(\hat{A}) & =\operatorname{span}\left\{\left(\omega_{\Lambda_{v}(x), \Lambda_{v}\left(x^{\prime}\right)} * \mathrm{id}\right)\left(W^{*}\right) \mid x, x^{\prime} \in A\right\} \\
& =\operatorname{span}\left\{\left(\omega_{\Lambda_{v}(x), \Lambda_{v}\left(x^{\prime}\right)} * \operatorname{id}\right)(W) \mid x, x^{\prime} \in A\right\} .
\end{aligned}
$$

For later use, we calculate the slices of $V$, which are defined similarly as those of $W^{*}$.

Lemma 3.4.4. Let $x, x^{\prime}, y, y^{\prime} \in A$. Then

$$
\begin{aligned}
\left(\omega_{\Lambda_{v}(x), \Lambda_{v}\left(x^{\prime}\right)} * \operatorname{id}\right)(V) \Lambda_{v}(y) & =\Lambda_{v}(\text { ay }), \text { where } a=\sum D^{-\frac{1}{2}}\left(x_{(2)}^{\prime} r\left(\psi\left(x^{*} x_{(1)}^{\prime}\right)\right)\right), \\
\left(\operatorname{id} * \omega_{\Lambda_{v}(y), \Lambda_{v}\left(y^{\prime}\right)}\right)(V) \Lambda_{v}(x) & =\Lambda_{v}(\check{c} * x), \text { where } c=S^{-1}\left(D^{-\frac{1}{2}}\left(y^{\prime} \theta\left(y^{*}\right)\right)\right)
\end{aligned}
$$

Proof. Without loss of generality, we assume $x$ to be homogeneous. Proceeding as in the proof of Lemma 3.4.1, we then find

$$
\begin{aligned}
\left(\lambda_{\Lambda_{v}(x)}^{\beta, \alpha}\right)^{*} V & \lambda_{\Lambda_{v}\left(x^{\prime}\right)}^{\widehat{\alpha}, \beta} \Lambda_{v}(y) \\
& \left.=\sum\left(\lambda_{\Lambda_{v}(x)}^{\beta, \alpha}\right)^{*}\left(\Lambda_{v}\left(\bar{D}^{\frac{1}{2}}\left(x_{(1)}^{\prime}\right)\right) \underset{\widetilde{\mu}}{\otimes} \Lambda_{v}\left(x_{(2)}^{\prime} y\right)\right) \quad \text { (Def. of } V\right) \\
& =\sum \alpha\left(\left\langle\Lambda_{v}(x) \mid \Lambda_{v}\left(\bar{D}^{\frac{1}{2}}\left(x_{(1)}^{\prime}\right)\right)\right\rangle_{\beta, \tilde{\mu}}\right) \Lambda_{v}\left(x_{(2)}^{\prime} y\right) \\
& =\sum \Lambda_{v}\left(r\left(\bar{\partial}_{x}\left(\psi\left(\bar{D}^{-\frac{1}{2}}(x)^{*} x_{(1)}^{\prime}\right)\right)\right) x_{(2)}^{\prime} y\right) \\
& =\sum \Lambda_{v}\left(D^{-\frac{1}{2}}\left(x_{(2)}^{\prime} r\left(\psi\left(x^{*} x_{(1)}^{\prime}\right)\right)\right) y\right),
\end{aligned}
$$




$$
\begin{aligned}
\left(\rho_{\Lambda_{v}(y)}^{\beta, \alpha}\right)^{*} V & \rho_{\Lambda_{v}\left(y^{\prime}\right)}^{\widehat{\alpha}, \beta} \Lambda_{v}(x) \\
& \left.=\sum\left(\rho_{\Lambda_{v}(y)}^{\beta, \alpha}\right)^{*}\left(\Lambda_{v}\left(x_{(1)}\right) \underset{\widetilde{\mu}}{\otimes} \Lambda_{v}\left(D^{\frac{1}{2}}\left(x_{(2)}\right) y^{\prime}\right)\right) \quad \text { (Def. of } V\right) \\
& =\sum \beta\left(\left\langle\Lambda_{v}(y) \mid \Lambda_{v}\left(D^{\frac{1}{2}}\left(x_{(2)}\right) y^{\prime}\right)\right\rangle_{\alpha, \tilde{\mu}}\right) \Lambda_{v}\left(x_{(1)}\right) \\
& =\sum \Lambda_{v}\left(s\left(\phi\left(D^{\frac{1}{2}}\left(x_{(2)}\right) y^{\prime} \theta\left(y^{*}\right)\right)\right) x_{(1)}\right) \\
& =\sum \Lambda_{v}\left(s\left(\phi\left(x_{(2)} D^{-\frac{1}{2}}\left(y^{\prime} \theta\left(y^{*}\right)\right)\right)\right) x_{(1)}\right) .
\end{aligned}
$$

3.5. The Hopf-von Neumann bimodules. We next show that the fundamental unitary $W$ is pseudo-multiplicative in the sense of [27] and therefore yields two Hopf-von Neumann bimodules, which are completions of $A$ and $\hat{A}$, respectively. First, we need further preliminaries.

The relative tensor product is functorial so that there exist bounded linear operators $S \underset{\tilde{\mu}}{\otimes} T \in \mathcal{L}\left(H_{\beta} \underset{\tilde{\mu}}{\otimes} \alpha\right)$ for all $S \in \beta(N)^{\prime}, T \in \alpha(N)^{\prime}$, as well as $S \underset{\widetilde{\mu}}{\otimes} T \in \mathcal{L}\left(H_{\alpha} \underset{\widetilde{\mu}}{\otimes} \widehat{\beta} H\right)$ for all $S \in \alpha(N)^{\prime}, T \in \widehat{\beta}(N)^{\prime}$, both times given by $\xi \underset{\tilde{\mu}}{\otimes} \eta \mapsto S \xi \underset{\tilde{\mu}}{\otimes} T \eta$.

In particular, the commuting representations $\alpha, \beta, \widehat{\alpha}, \widehat{\beta}$ yield six representations $\alpha \underset{\widetilde{\mu}}{\otimes}$ id, $\widehat{\alpha} \underset{\widetilde{\mu}}{\otimes}$ id, $\widehat{\beta} \underset{\widetilde{\mu}}{\otimes}$ id, id $\underset{\tilde{\mu}}{\otimes} \beta$, id $\underset{\tilde{\mu}}{\otimes} \widehat{\alpha}$, id $\underset{\widetilde{\mu}}{\otimes \beta}$ of $N$ on $H_{\beta} \underset{\tilde{\mu}}{\otimes_{\alpha}} H$, and further six

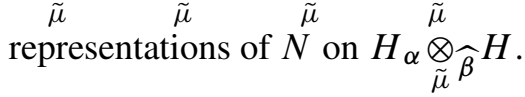

Lemma 3.5.1. The following relations hold for all $x \in N$ :

$$
\begin{aligned}
& W(\mathrm{id} \underset{\widetilde{\mu}}{\otimes} \widehat{\beta}(x))=(\beta(x) \underset{\widetilde{\mu}}{\otimes} \mathrm{id}) W, \quad W(\widehat{\alpha}(x) \underset{\widetilde{\mu}}{\otimes} \mathrm{id})=(\widehat{\alpha}(x) \underset{\widetilde{\mu}}{\otimes} \mathrm{id}) W, \\
& W(\widehat{\beta}(x) \underset{\widetilde{\mu}}{\otimes} \mathrm{id})=(\widehat{\beta}(x) \underset{\widetilde{\mu}}{\otimes} \mathrm{id}) W, \quad W(\alpha(x) \underset{\widetilde{\mu}}{\otimes} \mathrm{id})=(\underset{\tilde{\mu}}{\operatorname{id}} \underset{\widetilde{\alpha}}{\otimes} \alpha(x)) W, \\
& W(\operatorname{id} \underset{\tilde{\mu}}{\otimes} \beta(x))=(\operatorname{id} \underset{\tilde{\mu}}{\otimes} \beta(x)) W, \quad W(\operatorname{id} \underset{\tilde{\mu}}{\otimes} \widehat{\alpha}(x))=(\operatorname{id} \underset{\tilde{\mu}}{\underset{\alpha}{\alpha}}(x)) W .
\end{aligned}
$$

Proof. This follows immediately from the fact that $\pi_{\mu}(B) \subseteq N$ is weakly dense, the definition of $W$, and the formulas for $\alpha, \beta, \widehat{\alpha}, \widehat{\beta}$ given in Lemma 3.2.2.

The relative tensor product is associative in a natural sense. The intertwining relations for $W$ obtained above imply that all operators in the diagram below are well defined, 


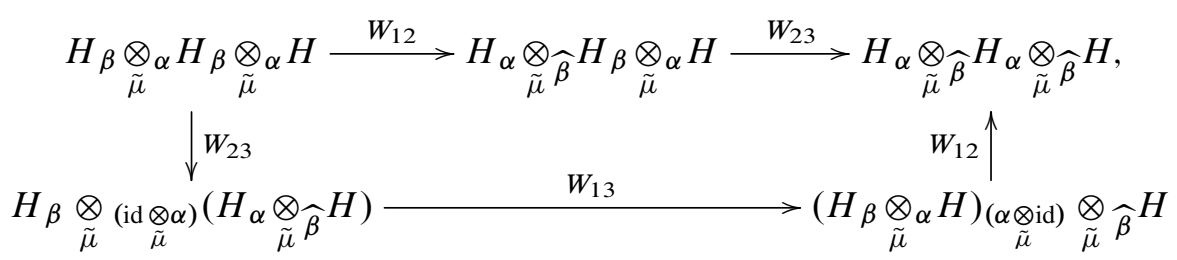

where $W_{12}=W \underset{\widetilde{\mu}}{\otimes} \mathrm{id}, W_{23}=\mathrm{id} \underset{\widetilde{\mu}}{\otimes}$, and $W_{13}$ acts on the first and third tensor factor; see [27] for details.

Lemma 3.5.2. Diagram (3.16) commutes, that is, $W_{23} W_{12}=W_{12} W_{13} W_{23}$.

Proof. A short calculation shows that the adjoints of both compositions are given by

$$
\begin{aligned}
\Lambda_{v}(x) \underset{\tilde{\mu}}{\otimes} \Lambda_{v}(y) & \underset{\widetilde{\mu}}{\otimes} \Lambda_{v}(z) \\
& \mapsto \sum \Lambda_{v}\left(z_{(1)} y_{(1)} x\right) \underset{\widetilde{\mu}}{\otimes} \Lambda_{v}\left(D^{\frac{1}{2}}\left(z_{(2)} y_{(2)}\right)\right) \underset{\widetilde{\mu}}{\otimes} \Lambda_{v}\left(D^{\frac{1}{2}}\left(z_{(3)}\right)\right) .
\end{aligned}
$$

Theorem 3.5.3. Let $(A, \Delta, \epsilon, S, \mu, \phi, \psi)$ be a measured multiplier $(B, \Gamma)$-Hopf *-algebroid such that $\mu$ admits a GNS-representation via bounded operators $\left(K, \Lambda_{\mu}, \pi_{\mu}\right)$ and its Radon-Nikodym cocycle has a positive square root in $M(B)$. Let $\tilde{\mu}$ be the weight on $N=\pi_{\mu}(B)^{\prime \prime}$ associated to the Hilbert algebra $\Lambda_{\mu}(B)$, let $\Lambda_{\nu}: A \rightarrow \mathcal{L}(H)$ be the GNS-map for $v=\mu \circ \phi=\mu \circ \psi$, and define $\alpha, \beta, \hat{\beta}: N \rightarrow \mathcal{L}(H)$ as in (3.4). Then the unitaries $W: H_{\beta} \underset{\tilde{\mu}}{\otimes_{\alpha}} H \rightarrow H_{\alpha}{\underset{\tilde{\mu}}{\widehat{\beta}}}_{\widehat{\beta}} H$ and $V: H_{\widehat{\alpha}} \otimes_{\widetilde{\mu}} \beta \rightarrow H_{\beta}{\underset{\widetilde{\mu}}{\alpha}}_{\alpha} H$ defined in Proposition 3.3 .2 and 3.3 .3 are pseudomultiplicative in the sense of [27].

Proof. The assertion on $W$ is just Lemma 3.5.1 and Lemma 3.5.2. For $V$, the proof is similar.

Definition 3.5.4. Let $(A, \Delta, \epsilon, S, \mu, \phi, \psi), W$ and $V$ as in Theorem 3.5.3. Then we call $W$ and $V$ the left and the right pseudo-multiplicative unitary of $(A, \Delta, \epsilon, S, \mu, \phi, \psi)$, respectively.

Recall from [26] that a Hopf-von Neumann bimodule over $(N, \tilde{\mu})$ is a von Neumann algebra $M$ acting on a Hilbert space $L$ together with faithful, nondegenerate, commuting normal representations $\gamma, \delta: N \rightarrow M$ and a non-degenerate, normal $*$-homomorphism $\Delta_{M}: M \rightarrow M_{\delta}{\underset{\tilde{\mu}}{\gamma}}_{\gamma} M$ such that $\Delta_{M} \circ \gamma=\gamma \underset{\widetilde{\mu}}{\otimes}$ id, $\Delta_{M} \circ \delta=\operatorname{id} \underset{\tilde{\mu}}{\otimes} \delta$ and $\left(\Delta_{M} \underset{\tilde{\mu}}{*} \mathrm{id}\right) \circ \Delta_{M}=\left(\mathrm{id} \underset{\tilde{\mu}}{*} \Delta_{M}\right)$, where $M_{\delta} \underset{\tilde{\mu}}{*} \gamma=\left(M^{\prime} \underset{\tilde{\mu}}{\otimes}\right.$ $\left.M^{\prime}\right)^{\prime} \subseteq \mathcal{L}\left(L_{\delta} \underset{\tilde{\mu}}{\otimes} \gamma\right.$, and $\Delta_{M} \underset{\tilde{\mu}}{*}$ id and id $\underset{\tilde{\mu}}{*} \Delta_{M}$ are suitably defined [16]. 
Lemma 3.5.5. The following relations hold:

$$
\begin{aligned}
& \alpha(N) \cup \beta(N) \subseteq \pi_{v}(A)^{\prime \prime} \subseteq \widehat{\beta}(N)^{\prime} \cap \widehat{\alpha}(N)^{\prime}, \\
& \hat{\beta}(N) \cup \alpha(N) \subseteq \rho(\hat{A})^{\prime \prime} \subseteq \beta(N)^{\prime} \cap \widehat{\alpha}(N)^{\prime}
\end{aligned}
$$

and

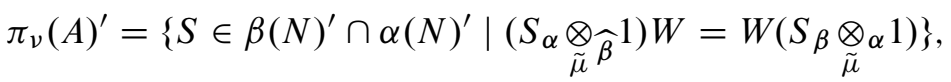

$$
\begin{aligned}
& \rho(\hat{A})^{\prime}=\left\{T \in \alpha(N)^{\prime} \cap \hat{\beta}(N)^{\prime} \mid\left(1_{\alpha} \underset{\tilde{\mu}}{\otimes} \widehat{\beta} T\right) W=W\left(1_{\beta} \underset{\widetilde{\mu}}{\underset{\tilde{\beta}}{\otimes}} T\right)\right\} .
\end{aligned}
$$

Proof. The inclusions in (3.17) follow from Lemma 3.2.2, non-degeneracy of $\pi_{v}(A)$ and $\rho(\hat{A})$ and equation (2.18). The equations (3.18) follow from (3.17) and Remark 3.4.3.

Using (3.17) and (3.18) and slightly abusing notation, we define faithful, normal, non-degenerate $*$-homomorphisms

$$
\begin{array}{ll}
\Delta: \pi_{\nu}(A)^{\prime \prime} \rightarrow \mathcal{L}\left(H_{\beta} \underset{\tilde{\mu}}{\left.\otimes_{\alpha} H\right),} \quad x \mapsto W^{*}(\underset{\widetilde{\mu}}{\operatorname{id}} x) W,\right. \\
\hat{\Delta}: \rho(\hat{A})^{\prime \prime} \rightarrow \mathcal{L}\left(H_{\alpha} \underset{\widetilde{\mu}}{\bigotimes_{\widehat{\beta}}} H\right), \quad y \mapsto \Sigma W(y \underset{\widetilde{\mu}}{\otimes} \mathrm{id}) W^{*} \Sigma .
\end{array}
$$

Theorem 3.5.6. Let $\mathcal{A}=(A, \Delta, \epsilon, S, \mu, \phi, \psi)$ be a measured multiplier $(B, \Gamma)$ Hopf $*$-algebroid such that $\mu$ admits a GNS-representation via bounded operators $\left(K, \Lambda_{\mu}, \pi_{\mu}\right)$ and the Radon-Nikodym cocycle of $\mu$ has a positive square root in $M(B)$. Let $\tilde{\mu}$ be the n.s.f. weight on $N=\pi_{\mu}(B)^{\prime \prime}$ associated to the Hilbert algebra $\Lambda_{\mu}(B)$ and let $\Lambda_{\nu}: A \rightarrow \mathcal{L}(H)$ be the GNS-map for $v=\mu \circ \phi=\mu \circ \psi$. Define $\pi_{\nu}: A \rightarrow \mathcal{L}(H), \rho: \hat{A} \rightarrow \mathcal{L}(H)$ as in (3.15), $\alpha, \beta, \hat{\beta}: N \rightarrow \mathcal{L}(H)$ as in (3.4) and

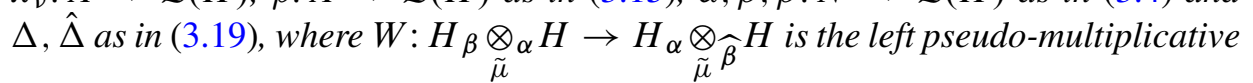
unitary of $\mathcal{A}$. Then $\left(\pi_{v}(A)^{\prime \prime}, \alpha, \beta, \Delta\right)$ and $\left(\rho(\hat{A})^{\prime \prime}, \widehat{\beta}, \alpha, \hat{\Delta}\right)$ are Hopf-von Neumann bimodules over $(N, \tilde{\mu})$.

Proof. The tuples $\left(\pi_{v}(A)^{\prime \prime}, \alpha, \beta, \Delta\right)$ and $\left(\rho(\hat{A})^{\prime \prime}, \widehat{\beta}, \alpha, \hat{\Delta}\right)$ are the Hopf-von Neumann bimodules associated to the pseudo-multiplicative unitary $W$. More precisely, the assertion follows from Proposition 10.3.10 and Theorem 10.3.11 in [23] and equation (3.18).

Definition 3.5.7. Let $(A, \Delta, \epsilon, S, \mu, \phi, \psi),\left(\pi_{\nu}(A)^{\prime \prime}, \alpha, \beta, \Delta\right)$ and $\left(\rho(\hat{A})^{\prime \prime}, \widehat{\beta}, \alpha, \hat{\Delta}\right)$ be as in Theorem 3.5.6. Then we call $\left(\pi_{v}(A)^{\prime \prime}, \alpha, \beta, \Delta\right)$ the Hopf-von Neumann bimodule of $(A, \Delta, \epsilon, S, \mu, \phi, \psi)$ and $\left(\rho(\hat{A})^{\prime \prime}, \widehat{\beta}, \alpha, \hat{\Delta}\right)$ the dual Hopf-von Neumann bimodule of $(A, \Delta, \epsilon, S, \mu, \phi, \psi)$. 
Theorem 3.5.6 above can also be deduced from the following explicit formulas for $\Delta$ and $\hat{\Delta}$ :

Lemma 3.5.8. For all $a, c, x, y \in A$,

$$
\begin{aligned}
\Delta\left(\pi_{v}(a)\right)\left(\Lambda_{v}(x) \underset{\tilde{\mu}}{\otimes} \Lambda_{v}(y)\right) & =\sum \Lambda_{v}\left(a_{(1)} x\right) \underset{\tilde{\mu}}{\otimes} \Lambda_{v}\left(D^{\frac{1}{2}}\left(a_{(2)}\right) y\right), \\
\hat{\Delta}(\rho(\hat{c}))\left(\Lambda_{v}(x) \underset{\tilde{\mu}}{\otimes} \Lambda_{v}(y)\right) & =\sum \Lambda_{v}\left(x_{(2)} r\left(\psi\left(S(c) x_{(1)} y_{(1)}\right)\right)\right) \underset{\tilde{\mu}}{\otimes} \Lambda_{v}\left(y_{(2)}\right),
\end{aligned}
$$

Proof. We calculate

$$
\begin{aligned}
& \Delta\left(\pi_{v}(a)\right) \sum \Lambda_{v}\left(y_{(1)} x\right) \underset{\tilde{\mu}}{\otimes} \Lambda_{v}\left(D^{\frac{1}{2}}\left(y_{(2)}\right)\right) \\
& =W^{*}\left(\underset{\tilde{\mu}}{\operatorname{id}} \pi_{v}(a)\right) W W^{*}\left(\Lambda_{v}(x) \underset{\tilde{\mu}}{\otimes} \Lambda_{v}(y)\right) \\
& =W^{*}\left(\Lambda_{\nu}(x) \underset{\tilde{\mu}}{\otimes} \Lambda_{\nu}(a y)\right) \\
& =\sum \Lambda_{v}\left(a_{(1)} y_{(1)} x\right) \underset{\tilde{\mu}}{\otimes} \Lambda_{v}\left(D^{\frac{1}{2}}\left(a_{(2)} y_{(2)}\right)\right), \\
& W^{*} \hat{\Delta}(\rho(\hat{c}))\left(\Lambda_{v}(x) \underset{\tilde{\mu}}{\otimes} \Lambda_{v}(y)\right) \\
& =(\rho(\hat{c}) \underset{\tilde{\mu}}{\otimes} \mathrm{id}) W^{*}\left(\Lambda_{v}(x) \underset{\tilde{\mu}}{\otimes} \Lambda_{v}(y)\right) \\
& =\sum \rho(\hat{c}) \Lambda_{v}\left(y_{(1)} x\right) \underset{\tilde{\mu}}{\otimes} \Lambda_{v}\left(D^{\frac{1}{2}}\left(y_{(2)}\right)\right) \\
& =\sum \Lambda_{v}\left(y_{(2)} x_{(2)} r\left(\psi\left(S(c) y_{(1)} x_{(1)}\right)\right)\right) \underset{\tilde{\mu}}{\otimes} \Lambda_{v}\left(D^{\frac{1}{2}}\left(y_{(3)}\right)\right) \\
& =W^{*} \sum \Lambda_{v}\left(x_{(2)} r\left(\psi\left(S(c) y_{(1)} x_{(1)}\right)\right)\right) \underset{\tilde{\mu}}{\otimes} \Lambda_{v}\left(y_{(2)}\right) .
\end{aligned}
$$

Remark 3.5.9. Under the identification (3.9), for all homogeneous $a, x, y \in A$ and $\zeta \in K$

$$
\Delta\left(\pi_{v}(a)\right)\left(\Lambda_{v}(x) \otimes \zeta \otimes \Lambda_{v}(y)\right)=\sum \Lambda_{v}\left(a_{(1)} x\right) \otimes U_{\bar{\partial}_{(1)}} \zeta \otimes \Lambda_{v}\left(a_{(2)} y\right),
$$

where $a_{(1)}$ is assumed to be homogeneous without loss of generality.

3.6. The Hopf $C^{*}$-bimodules. The fundamental unitary $W$ is a regular $C^{*}$ pseudo-multiplicative unitary in the sense of [24], and therefore yields Hopf $C^{*}$. bimodules which are completions of $A$ and $\hat{A}$. To prove this, we again need some preliminaries concerning the relative tensor product in the setting of $C^{*}$-algebras; for details, see [22] and [24]. The construction is parallel to the von Neumann-algebraic setting and differs mainly in notation. 
As before, let $\mathfrak{b}=\left(K,\left[\pi_{\mu}(B)\right],\left[\pi_{\mu}(B)\right]\right)$. The relative tensor product $H_{E_{\psi}^{\dagger} \mathfrak{b}}^{\otimes} E_{\phi}^{\dagger} H$ of the $C^{*}$-b -modules $\left(H, E_{\psi}^{\dagger}\right)$ and $\left(H, E_{\phi}^{\dagger}\right)$ is the separated completion of the algebraic tensor product $E_{\psi}^{\dagger} \otimes K \otimes E_{\phi}^{\dagger}$ with respect to the sesquilinear form given by

$$
\left\langle\xi \otimes \zeta \otimes \eta \mid \xi^{\prime} \otimes \zeta^{\prime} \otimes \eta^{\prime}\right\rangle=\left\langle\zeta \mid\left(\xi^{*} \xi^{\prime}\right)\left(\eta^{*} \eta^{\prime}\right) \zeta^{\prime}\right\rangle
$$

It can be regarded as a twofold internal tensor product of Hilbert $C^{*}$-modules and identified with certain separated completions $E_{\psi}^{\dagger} \theta_{\alpha} H$ and $H_{\beta} \otimes E_{\phi}^{\dagger}$ of the algebraic tensor products $E_{\psi}^{\dagger} \otimes H$ and $H \otimes E_{\phi}^{\dagger}$, respectively, such that

$$
E_{\psi}^{\dagger} \otimes_{\alpha} H \cong H_{E_{\psi}^{\dagger}} \otimes_{\mathfrak{b}} E_{\phi}^{\dagger} H \cong H_{\beta} \otimes E_{\phi}^{\dagger}, \quad \xi \otimes \eta \zeta \equiv \xi \otimes \zeta \otimes \eta \equiv \xi \zeta \otimes \eta .
$$

Comparing the sesquilinear forms (3.7) with (3.20) and using (3.6), one finds that there exists an isomorphism

$$
H_{\beta} \underset{\tilde{\mu}}{\otimes} \alpha \cong H_{E_{\psi}^{\dagger}}^{\dagger} \underset{\mathfrak{b}}{\otimes} E_{\phi}^{\dagger} H, \quad \Lambda_{v}(x) \otimes \zeta \otimes \Lambda_{v}(y) \equiv \Lambda_{\psi}^{\dagger}(x) \otimes \zeta \otimes \Lambda_{\phi}^{\dagger}(y) .
$$

For each $\xi \in E_{\psi}^{\dagger}$ and $\eta \in E_{\phi}^{\dagger}$, there exist bounded linear operators

$$
|\xi\rangle_{1}: H \rightarrow H_{E_{\psi}^{\dagger} \underset{\mathfrak{b}}{\otimes} E_{\phi}^{\dagger}}^{\otimes} H, \eta^{\prime} \mapsto \xi \otimes \eta^{\prime}, \quad|\eta\rangle_{2}: H \rightarrow H_{E_{\psi}^{\dagger}}^{\dagger} \underset{\mathfrak{b}}{\otimes} E_{\phi}^{\dagger} H, \xi^{\prime} \mapsto \xi^{\prime} \otimes \eta .
$$

We denote their adjoints by $\left\langle\left.\xi\right|_{1}\right.$ and $\left\langle\left.\eta\right|_{2} \text {, respectively, and write } \mid E_{\psi}^{\dagger}\right\rangle_{1}=\left\{|\xi\rangle_{1}\right.$ : $\left.\xi \in E_{\psi}^{\dagger}\right\},\left|E_{\phi}^{\dagger}\right\rangle_{2}=\left\{|\eta\rangle_{2}: \eta \in E_{\phi}^{\dagger}\right\}$ et cetera. Comparing with (3.13), we see that under the identification (3.22), $\lambda_{\Lambda_{\nu}(x)}^{\widehat{\alpha}, \beta} \equiv\left|\Lambda_{\psi}^{\dagger}(x)\right\rangle_{1}$ and $\rho_{\Lambda_{\nu}(y)}^{\widehat{\alpha}, \beta} \equiv\left|\Lambda_{\phi}^{\dagger}(y)\right\rangle_{2}$ for all $x, y \in A$.

Replacing $E_{\psi}^{\dagger}$ and $E_{\phi}^{\dagger}$ by $E_{\phi}^{\dagger}$ and $E_{\phi}$, respectively, one similarly defines the relative tensor product $H_{E_{\phi}^{\dagger}}^{\dagger} \underset{\mathfrak{b}}{\otimes} E_{\phi} H$ with a canonical isomorphism $H_{E_{\phi}^{\dagger}} \underset{\mathfrak{b}}{\otimes_{E_{\phi}}} H \cong$

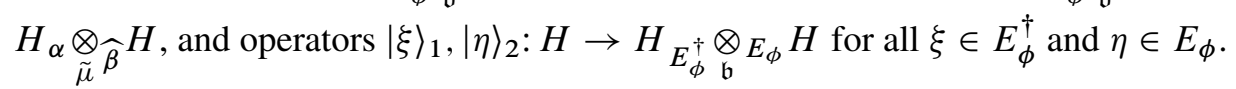

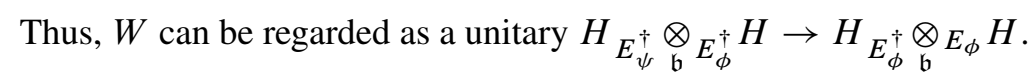


Lemma 3.6.1. For all $x, x^{\prime}, y, y^{\prime} \in A$ and $\gamma \in\{\alpha, \beta, \widehat{\beta}\}, \gamma^{\prime} \in\{\alpha, \widehat{\alpha}, \beta\}$,

$$
\begin{aligned}
& \Lambda_{v}(x) \underset{\tilde{\mu}}{\otimes} \Lambda_{v}(y) \in D\left(\left(H_{\beta} \underset{\tilde{\mu}}{\otimes_{\alpha} H}\right)_{\mathrm{id}} \underset{\tilde{\mu}}{\otimes \gamma}, \tilde{\mu}\right), \\
& R_{\Lambda_{\nu}(x) \underset{\tilde{\mu}}{\operatorname{id}} \underset{\tilde{\mu}}{\otimes}, \tilde{\mu}}=\lambda_{\Lambda_{\nu}(y)}^{\beta, \alpha} R_{\Lambda_{v}(y)}^{\gamma, \tilde{\mu}}=\left|\Lambda_{\psi}^{\dagger}(x)\right\rangle_{1} R_{\Lambda_{\nu}(y)}^{\gamma, \tilde{\mu}},
\end{aligned}
$$

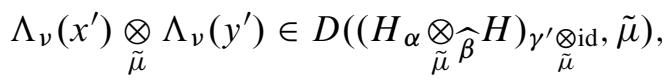

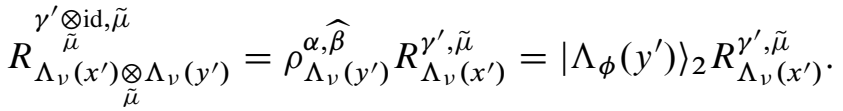

Proposition 3.6.2. The following equations for subspaces of $\mathcal{L}\left(H, H_{E_{\psi}^{\dagger}}^{\dagger} \underset{\mathfrak{b}}{\otimes} E_{\phi}^{\dagger} H\right)$ hold:

$$
\begin{aligned}
W\left[\left|E_{\psi}^{\dagger}\right\rangle_{1} E_{\phi}\right] & =\left[\left|E_{\phi}\right\rangle_{2} E_{\psi}^{\dagger}\right], & W\left[\left|E_{\phi}^{\dagger}\right\rangle_{2} E_{\psi}\right] & =\left[\left|E_{\phi}\right\rangle_{2} E_{\psi}\right], \\
W\left[\left|E_{\phi}^{\dagger}\right\rangle_{2} E_{\phi}\right] & =\left[\left|E_{\phi}\right\rangle_{2} E_{\phi}\right], & W\left[\left|E_{\phi}^{\dagger}\right\rangle_{2} E_{\phi}^{\dagger}\right] & =\left[\left|E_{\phi}^{\dagger}\right\rangle_{1} E_{\phi}^{\dagger}\right], \\
W\left[\left|E_{\psi}^{\dagger}\right\rangle_{1} E_{\psi}^{\dagger}\right] & =\left[\left|E_{\phi}^{\dagger}\right\rangle_{1} E_{\psi}^{\dagger}\right], & W\left[\left|E_{\psi}^{\dagger}\right\rangle_{1} E_{\psi}\right] & =\left[\left|E_{\phi}^{\dagger}\right\rangle_{1} E_{\psi}\right] .
\end{aligned}
$$

Proof. We only prove the first equation; the others follow similarly:

$$
\begin{aligned}
& W\left[\left|E_{\psi}^{\dagger}\right\rangle_{1} E_{\phi}\right]=\left[\left\{W R_{\omega}^{\operatorname{id} \underset{\tilde{\mu}}{\otimes}, \tilde{\mu}}: \omega \in \Lambda_{v}(A) \underset{\widetilde{\mu}}{\otimes} \Lambda_{v}(A)\right\}\right] \quad \text { (Lem. 3.6.1 and (3.2.3)) } \\
& =\left[\left\{R_{W \omega}^{\substack{\otimes \\
\tilde{\mu} \mathrm{id}, \tilde{\mu}}}: \omega \in \Lambda_{v}(A) \underset{\widetilde{\mu}}{\otimes} \Lambda_{v}(A)\right\}\right] \quad \text { (Lem. 3.5.1) }
\end{aligned}
$$

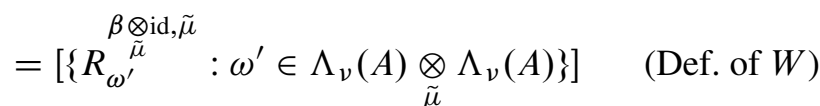

$$
\begin{aligned}
& =\left[\left|E_{\phi}\right\rangle_{2} E_{\psi}^{\dagger}\right] \text { (Lem. 3.6.1 and 3.2.3) }
\end{aligned}
$$

Theorem 3.6.3. Let $\mathcal{A}=(A, \Delta, \epsilon, S, \mu, \phi, \psi)$ be a measured multiplier $(B, \Gamma)$ Hopf $*$-algebroid such that $\mu$ admits a GNS-representation via bounded operators $\left(K, \Lambda_{\mu}, \pi_{\mu}\right)$ and the Radon-Nikodym cocycle of $\mu$ has a positive square root in $M(B)$. Let $\mathfrak{b}=\left(K,\left[\pi_{\mu}(B)\right],\left[\pi_{\mu}(B)\right]\right)$, let $\Lambda_{v}: A \rightarrow \mathcal{L}(H)$ be the GNS-map of $v=\mu \circ \phi=\mu \circ \psi$ and define $E_{\phi}, E_{\phi}^{\dagger}, E_{\psi}, E_{\psi}^{\dagger} \subseteq \mathcal{L}(K, H)$ as in (3.3). Then the left and the right pseudo-multiplicative unitary $W$ and $V$ of $\mathcal{A}$, regarded as operators $H_{E_{\psi}^{\dagger} \underset{\mathfrak{b}}{\otimes} E_{\phi}^{\dagger}} H \rightarrow H_{E_{\phi}^{\dagger}}^{\otimes_{\mathfrak{b}}} E_{\phi} H$ and $H_{E_{\psi}} \underset{\mathfrak{b} E_{\psi}^{\dagger}}{\otimes} H \rightarrow H_{E_{\psi}^{\dagger}} \underset{\mathfrak{b}}{\otimes} E_{\phi}^{\dagger} H$ as above, are $C^{*}$-pseudo-multiplicative unitaries in the sense of [24].

Proof. The assertion on $W$ is Proposition 3.6.2 and Lemma 3.5.2. For $V$, the proof is similar. 
Proposition 3.6.4. $W$ and $V$ are regular in the sense that $\left[\left\langle\left. E_{\phi}^{\dagger}\right|_{1} W \mid E_{\phi}^{\dagger}\right\rangle_{2}\right]=$ $\left[E_{\phi}^{\dagger}\left(E_{\phi}^{\dagger}\right)^{*}\right] \subseteq \mathcal{L}(H)$ and $\left[\left\langle\left. E_{\psi}^{\dagger}\right|_{1} V \mid E_{\psi}^{\dagger}\right\rangle_{2}\right]=\left[E_{\psi}^{\dagger}\left(E_{\psi}^{\dagger}\right)^{*}\right] \subseteq \mathcal{L}(H)$.

Proof. Let $x, x^{\prime}, y \in A$. Then $\Lambda_{\phi}^{\dagger}(y) \Lambda_{\phi}^{\dagger}(x)^{*} \Lambda_{v}\left(y^{\prime}\right)=\Lambda_{v}\left(r\left(\phi\left(y^{\prime} \theta\left(x^{*}\right)\right)\right) y\right)$ by Lemma 3.2.1 and

$$
\begin{aligned}
\left\langle\left.\Lambda_{\phi}^{\dagger}(y)\right|_{2}\right. & W^{*}\left|\Lambda_{\phi}^{\dagger}(x)\right\rangle_{1} \Lambda_{v}\left(y^{\prime}\right) \\
& =\left(\rho_{\Lambda_{v}(y)}^{\beta, \alpha}\right)^{*} W^{*}\left(\Lambda_{v}(x) \underset{\widetilde{\mu}}{\underset{\sim}{\otimes}} \Lambda_{v}\left(y^{\prime}\right)\right) \\
& =\sum \beta\left(\left\langle\Lambda_{v}(y) \mid \Lambda_{v}\left(D^{\frac{1}{2}}\left(y_{(2)}^{\prime}\right)\right)\right\rangle_{\alpha, \tilde{\mu}}\right) \Lambda_{v}\left(y_{(1)}^{\prime} x\right) \\
& =\sum \Lambda_{v}\left(s\left(\phi\left(D^{\frac{1}{2}}\left(y_{(2)}^{\prime}\right) \theta\left(y^{*}\right)\right)\right) y_{(1)}^{\prime} x\right) \\
& =\sum \Lambda_{v}\left(s\left(\phi\left(y_{(2)}^{\prime} z\right)\right) y_{(1)}^{\prime} x\right) \\
& =\sum \Lambda_{v}\left(r\left(\phi\left(y^{\prime} z_{(2)}\right)\right) S^{-1}\left(z_{(1)}\right) x\right) .
\end{aligned}
$$$$
\text { with } z:=D^{-\frac{1}{2}}\left(\theta\left(y^{*}\right)\right)
$$

Since the maps $\theta, D^{-\frac{1}{2}}, S$ and $T_{3}$ are bijections, we can conclude

$$
\left[\left\{\Lambda_{\phi}^{\dagger}(y) \Lambda_{\phi}^{\dagger}(x)^{*}: x, y \in A\right\}\right]=\left[\left\{\left\langle\left.\Lambda_{\phi}^{\dagger}(x)\right|_{2} W^{*} \mid \Lambda_{\phi}^{\dagger}(y)\right\rangle_{1}: x, y \in A\right\}\right] .
$$

The assertion on $V$ follows from a similar calculation.

Recall from [24] that a $\operatorname{Hopf} C^{*}$-bimodule over $\mathfrak{b}$ consists of a $C^{*}-(\mathfrak{b}, \mathfrak{b})$-module $(L, E, F)$, a non-degenerate $C^{*}$-algebra $C \subseteq \mathcal{L}(L)$ satisfying $\rho_{E}\left(\pi_{\mu}(B)\right) \subseteq M(C)$ and $\rho_{F}\left(\pi_{\mu}(B)\right) \subseteq M(C)$, and a non-degenerate $*$-homomorphism $\Delta_{C}: C \rightarrow$ $C_{F}{ }_{b}{ }_{E} C$ that is co-associative and compatible with $E$ and $F$ in a suitable sense, where

$$
\begin{aligned}
C_{F}{ }_{\mathfrak{b}}^{*} C=\left\{T \in \mathcal{L}\left(L_{F} \underset{\mathfrak{b}}{\otimes_{E}} L\right): T|F\rangle_{1}+T^{*}|F\rangle_{1} \subseteq\left[|F\rangle_{1} C\right]\right. \\
\\
\left.\quad \text { and } T|E\rangle_{2}+T^{*}|E\rangle_{2} \subseteq\left[|E\rangle_{2} C\right]\right\}
\end{aligned}
$$

is the fiber product of $C$ with itself relative to $F$ and $E$.

Theorem 3.6.5. Let $\mathcal{A}=(A, \Delta, \epsilon, S, \mu, \phi, \psi)$ be a measured multiplier $(B, \Gamma)$ Hopf $*$-algebroid such that $\mu$ admits a GNS-representation via bounded operators $\left(K, \Lambda_{\mu}, \pi_{\mu}\right)$ and its Radon-Nikodym cocycle has a positive square root in $M(B) . \quad$ Let $\mathfrak{b}=\left(K,\left[\pi_{\mu}(B)\right],\left[\pi_{\mu}(B)\right]\right)$, let $\Lambda_{\nu}: A \rightarrow \mathcal{L}(H)$ be the GNSmap for $v=\mu \circ \phi=\mu \circ \psi$, let $\left(\pi_{\nu}(A)^{\prime \prime}, \alpha, \beta, \Delta\right)$ be the Hopf-von Neumann bimodule of $\mathcal{A}$, let $\left(\rho(\hat{A})^{\prime \prime}, \hat{\beta}, \alpha, \hat{\Delta}\right)$ be the dual Hopf-von Neumann bimodule of $\mathcal{A}$, define $E_{\phi}, E_{\phi}^{\dagger}, E_{\psi}, E_{\psi}^{\dagger} \subseteq \mathcal{L}(K, H)$ as in (3.3) and regard $\Delta$

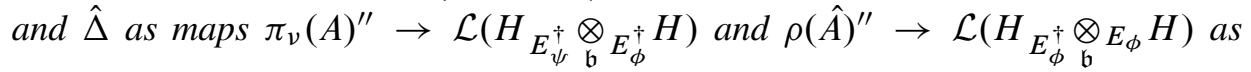
above. Then $\left(\left(H, E_{\phi}^{\dagger}, E_{\psi}^{\dagger}\right),\left[\pi_{\nu}(A)\right],\left.\Delta\right|_{\left[\pi_{v}(A)\right]}\right)$ and $\left(\left(H, E_{\psi}, E_{\phi}^{\dagger}\right),[\rho(\hat{A})],\left.\hat{\Delta}\right|_{[\rho(\hat{A})]}\right)$ are Hopf $C^{*}$-bimodules over $\mathfrak{b}$. 
Proof. By Theorem 3.6.3 and Proposition 3.6.4, the left pseudo-multiplicative unitary $W$ of $\mathcal{A}$, regarded as a unitary $H_{E_{\psi}^{\dagger} \underset{\mathfrak{b}}{\dagger} E_{\phi}^{\dagger}} H \rightarrow H_{E_{\phi}^{\dagger}}^{\underset{\mathfrak{b}}{\otimes_{E_{\phi}}}} H$, is a regular $C^{*}$ pseudo-multiplicative unitary. By [24], the latter gives rise to two Hopf $C^{*}$-bimodules $\left(\left(H, E_{\phi}^{\dagger}, E_{\psi}^{\dagger}\right),\left[\left\langle\left. E_{\phi}\right|_{2} W \mid E_{\phi}^{\dagger}\right\rangle_{2}\right], \Delta\right)$ and $\left(\left(H, E_{\psi}, E_{\psi}^{\dagger}\right),\left[\left\langle\left. E_{\phi}^{\dagger}\right|_{1} W \mid E_{\psi}^{\dagger}\right\rangle_{1}\right], \hat{\Delta}\right)$. Finally, by Lemma 3.4.1, $\left[\left\langle\left. E_{\phi}^{\dagger}\right|_{1} W \mid E_{\psi}^{\dagger}\right\rangle_{1}\right]=[\rho(\hat{A})]$ and $\left[\left\langle\left. E_{\phi}\right|_{2} W \mid E_{\phi}^{\dagger}\right\rangle_{2}\right]=[\lambda(\hat{A})]$.

3.7. The measured quantum groupoid. To obtain a measured quantum groupoid, we finally extend $v, \phi, \psi$ to normal, semi-finite, faithful weights on the level of von Neumann algebras. We impose the following simplifying assumptions:

(A3) the bimodule ${ }_{r} A_{s}$ is proper in the sense that $r(B) s(B) \subseteq A$.

(A4) There exists a net $\left(u_{i}\right)_{i}$ in $B$ that is truncating for $\mu$ in the sense that $\left(\pi_{\mu}\left(u_{i}\right)\right)_{i}$ is a net of positive elements in the unit ball of $\pi_{\mu}(B)$ that converges in $M\left(\left[\pi_{\mu}(B)\right]\right)$ strictly to 1 and such that $\left(\pi_{\mu}\left(u_{i}^{2}\right)\right)_{i}$ is increasing.

Note that a net $\left(u_{i}\right)_{i}$ as in (A4) exists always if we drop the condition that $\left(\pi_{\mu}\left(u_{i}^{2}\right)\right)_{i}$ should be increasing.

Let us also note that in the bi-measured case where $\phi, \psi$ and $v$ arise from a biintegral $h$ on $(A, \Delta)$, the extensions of $\phi, \psi, v$ and the invariance of these extensions can be proved quite easily, see Remark 3.7.5 and 3.7.10.

For the extension of $v$, we do not need the assumptions (A3) and (A4), but use the modular automorphism $\theta$ for $v$ obtained in Theorem 2.6.5, the theory of Hilbert algebras [20], and results of Kustermans and van Daele [11].

Lemma 3.7.1. $\Lambda_{v}(A) \subseteq H$ is a Hilbert algebra with respect to the $*$-algebra structure inherited from $A$.

Proof. The multiplication $\Lambda_{v}(y) \mapsto \Lambda_{v}(x y)$ is bounded for each $x \in A$ by Theorem 3.4.2, and the involution $\Lambda_{v}(x) \mapsto \Lambda_{v}\left(x^{*}\right)$ is pre-closed because

$$
\left\langle\Lambda_{v}(x) \mid \Lambda_{v}\left(y^{*}\right)\right\rangle=v\left(x^{*} y^{*}\right)=v\left(y^{*} \theta\left(x^{*}\right)\right)=\left\langle\Lambda_{v}(y) \mid \Lambda_{v}\left(\theta\left(x^{*}\right)\right)\right\rangle
$$

for all $x, y \in A$. 
The general theory of Hilbert algebras [20] now yields

- $M=\pi_{v}(A)^{\prime \prime} \subseteq \mathcal{L}(H)$ as the associated von Neumann algebra,

- a n.s.f. weight $\tilde{v}$ on $M$ such that $\tilde{v}\left(\pi_{v}\left(a^{*} a\right)\right)=\left\langle\Lambda_{v}(a) \mid \Lambda_{v}(a)\right\rangle=v\left(a^{*} a\right)$ for all $a \in A$,

- a left ideal $\mathfrak{N}_{\tilde{v}}:=\left\{x \in M: \tilde{v}\left(x^{*} x\right)<\infty\right\} \subseteq M$ of square-integrable elements,

- a closed map $\Lambda_{\tilde{v}}: \mathfrak{N}_{\tilde{v}} \rightarrow H$ such that $\left(H, \Lambda_{\tilde{v}}, \operatorname{id}_{M}\right)$ is a GNS-representation for $\tilde{v}$; this is the closure of the map $\pi_{v}(A) \rightarrow H$ given by $\pi_{v}(a) \rightarrow \Lambda_{v}(a)$;

- the usual objects $J_{\tilde{v}}, \Delta_{\tilde{v}}, \sigma^{\tilde{v}}, \mathcal{T}_{\tilde{v}}, \ldots$ of Tomita-Takesaki theory.

The modular automorphism $\theta$ is related to the modular automorphism group $\sigma^{\tilde{v}}$ as follows:

Proposition 3.7.2. $\pi_{v}(A) \subseteq \mathcal{T}_{\tilde{v}}$ and $\sigma_{n i}^{\tilde{\nu}}\left(\pi_{v}(a)\right)=\pi_{v}\left(\theta^{-n}(a)\right)$ for all $a \in A$, $n \in \mathbb{Z}$.

Proof. Use the arguments in [12, §3], in particular from Lemma 3.16 till Proposition 3.22 .

Let $A^{\theta}:=\{a \in A: \theta(a)=a\} \subseteq A$. Note that this space is a $*$-subalgebra and, by (A3), contains $r(B) s(B)$.

\section{Lemma 3.7.3.}

i) $\sigma^{\tilde{v}}$ acts trivially on $\pi_{v}\left(A^{\theta}\right)^{\prime \prime}$, in particular on $\alpha(N)$ and $\beta(N)$.

ii) $J_{\tilde{v}} \alpha(x)^{*} J_{\tilde{v}}=\widehat{\beta}(x)$ and $J_{\tilde{v}} \beta(x)^{*} J_{\tilde{v}}=\widehat{\alpha}(x)$ for all $x \in N$.

Proof. i) The first assertion follows from the fact that $\sigma_{t}^{\tilde{\nu}}(x)=\Delta_{\tilde{\nu}}^{i t} x \Delta_{\tilde{\nu}}^{-i t}$ and $\Delta_{\tilde{v}}^{-1} x \Delta_{\tilde{v}}=x$ for each $x \in \pi_{\tilde{v}}\left(A^{\theta}\right)$ by Proposition 3.7.2, and the second assertion follows from the fact that $\sigma_{t}^{\tilde{v}}$ is normal for all $t \in \mathbb{R}$ and acts trivially on $\pi_{v}(r(B) s(B))$.

ii) Combine i) and Lemma 3.2.2.

Proposition 3.7.4. There exist unique n.s.f. weights $T_{L}$ from $M$ to $\alpha(N)$ and $T_{R}$ from $M$ to $\beta(N)$ such that $\tilde{\mu} \circ \alpha^{-1} \circ T_{L}=\tilde{v}=\tilde{\mu} \circ \beta^{-1} \circ T_{R}$.

Proof. This follows from Lemma 3.7.3 i) and [18, 10.1] or [20, IX Theorem 4.18].

We thus obtain extensions $\tilde{\phi}:=\alpha^{-1} \circ T_{L}$ and $\tilde{\psi}:=\beta^{-1} \circ T_{R}$ of $\phi$ and $\psi$.

Remark 3.7.5. Assume that $\phi=(\mathrm{id} \otimes \mu) \circ h$ and $\psi=(\mu \otimes \mathrm{id}) \circ h$ for a normalized bi-integral $h$ on $(A, \Delta)$. Then the map $\Lambda_{\mu}(B) \otimes \Lambda_{\mu}(B) \rightarrow \Lambda_{\nu}(A)$ given by $\Lambda_{\mu}(b) \otimes$ $\Lambda_{\mu}\left(b^{\prime}\right) \mapsto \Lambda_{\nu}\left(r(b) s\left(b^{\prime}\right)\right)$ extends to an isometry $\iota: K \otimes K \rightarrow H$, and a short calculation shows that $\iota^{*} \pi_{\nu}(a) \iota=\left(\pi_{\mu} \otimes \pi_{\mu}\right)(h(a))$ for all $a \in A$. We therefore get a positive, normal, linear extension $\tilde{h}: M \rightarrow N, x \mapsto \iota^{*} x \iota$, of $h$, and thereby the desired extensions $\tilde{\phi}=(\operatorname{id} \bar{\otimes} \tilde{\mu}) \circ \tilde{h}, \tilde{\psi}=(\tilde{\mu} \bar{\otimes}$ id $) \circ \tilde{h}$ and $\tilde{v}=(\tilde{\mu} \bar{\otimes} \tilde{\mu}) \circ \tilde{h}$. 
Recall that an element $\xi \in H$ is right-bounded with respect to the Hilbert algebra $\Lambda_{v}(A)$ if there exists an operator $R_{\xi} \in \mathcal{L}(H)$ such that $\pi_{v}(a) \xi=R_{\xi} \Lambda_{v}(a)$ for all $a \in A$. Note that then $R_{\xi} \in M^{\prime}$.

\section{Lemma 3.7.6.}

i) If $x \in A^{\theta}$, then $\Lambda_{v}(x) \in H$ is right-bounded, $R_{\Lambda_{v}(x)}=J_{v} \pi_{v}(x)^{*} J_{v}$ and $\left\|R_{\Lambda_{v}(x)}\right\|=\left\|\pi_{v}(x)\right\|$.

ii) If $x \in A^{\theta} \cap r(B)^{\prime}$, then $\pi_{v}(a) \Lambda_{\phi}(x)=R_{\Lambda_{v}(x)} \Lambda_{\phi}(a)$ for all $a \in A$.

iii) If $a \in A$ and $\xi \in K$ is right-bounded with respect to $\Lambda_{\mu}(B)$, then $\Lambda_{\phi}(a) \xi=$ $\widehat{\beta}\left(R_{\xi}\right) \Lambda_{v}(a)$.

Proof. i) For all $x \in A^{\theta}, a \in A$, we have $\pi_{v}(a) \Lambda_{v}(x)=\Lambda_{v}(a x)=J_{v} \pi_{v}(x)^{*} J_{v} \Lambda_{v}(a)$.

ii) For all $x \in A^{\theta} \cap r(B)^{\prime}, a \in A, b \in B$,

$$
\begin{aligned}
\pi_{v}(a) \Lambda_{\phi}(x) \Lambda_{\mu}(b)=\Lambda_{v}(\operatorname{axr}(b)) & =\Lambda_{v}(\operatorname{ar}(b) x) \\
& =\pi_{v}(\operatorname{ar}(b)) \Lambda_{v}(x) \\
& =R_{\Lambda_{v}(x)} \Lambda_{v}(\operatorname{ar}(b))=R_{\Lambda_{v}(x)} \Lambda_{\phi}(a) \Lambda_{\mu}(b) .
\end{aligned}
$$

iii) If $a \in A$ and $\xi=\Lambda_{\mu}(b)$ for some $b \in B$, then $R_{\xi}=\pi_{\mu}(b)$ and $\Lambda_{\phi}(a) \xi=$ $\Lambda_{v}(\operatorname{ar}(b))=\widehat{\beta}\left(\pi_{\mu}(b)\right) \Lambda_{v}(a)$. Now, the assertion follows for all right-bounded $\xi$ because $\Lambda_{\mu}(B)$ is a core for $\Lambda_{\tilde{\mu}}$ and the right-bounded elements coincide with $\Lambda_{\tilde{\mu}}\left(\mathfrak{N}_{\tilde{\mu}}\right)$.

To prove Theorem 3.7.9, we construct increasing approximations of the weights $\tilde{\mu}, \tilde{v}, \tilde{\phi}, \tilde{\psi}$ by bounded positive maps, using an approximate unit $\left(u_{i}\right)_{i}$ in $B$ with the properties assumed in (A4). Let $u_{i, j}:=r\left(u_{i}\right) s\left(u_{j}\right) \in A$, and define for all $i, j$ bounded, normal, positive, linear maps

$$
\begin{aligned}
\mu_{i}: N \rightarrow \mathbb{C}, & x \mapsto\left\langle\Lambda_{\mu}\left(u_{i}\right) \mid x \Lambda_{\mu}\left(u_{i}\right)\right\rangle, \\
v_{i, j}: M \rightarrow \mathbb{C}, & x \mapsto\left\langle\Lambda_{v}\left(u_{i, j}\right) \mid x \Lambda_{v}\left(u_{i, j}\right)\right\rangle, \\
\phi_{i, j}: M \rightarrow N, & x \mapsto \Lambda_{\phi}\left(u_{i, j}\right)^{*} x \Lambda_{\phi}\left(u_{i, j}\right), \\
\psi_{i, j}: M \rightarrow N, & x \mapsto \Lambda_{\psi}\left(u_{i, j}\right)^{*} x \Lambda_{\psi}\left(u_{i, j}\right) .
\end{aligned}
$$

Given a net $\left(\lambda_{\kappa}\right)_{\kappa}$ of real numbers, we write $\left(\lambda_{\kappa}\right)_{\kappa} \nearrow \lambda$ if it is increasing and converges to $\lambda$. Likewise, given a von Neumann algebra $C$ with a net $\left(\omega_{\kappa}\right)_{\kappa}$ in $C_{*}^{+}$ and a n.s.f. weight $\omega$, we write $\left(\omega_{\kappa}\right)_{\kappa} \nearrow \omega$ if $\omega_{\kappa}\left(x^{*} x\right) \nearrow \omega\left(x^{*} x\right)$ for all $x \in C$.

\section{Proposition 3.7.7.}

i) $\left(\mu_{i}\right)_{i} \nearrow \tilde{\mu}$ and $\left(v_{i, j}\right)_{i, j} \nearrow \tilde{v}$;

ii) $\left(v \circ \phi_{i, j}\right)_{i, j} \nearrow v \circ \tilde{\phi}$ and $\left(v \circ \psi_{i, j}\right)_{i, j} \nearrow v \circ \tilde{\psi}$ for all $v \in N_{*}^{+}$. 
Proof. i) We only prove the assertion concerning $\tilde{v}$.

Let $\xi_{i, j}:=\Lambda_{v}\left(u_{i, j}\right)$ and $R_{i, j}:=R_{\xi_{i, j}}=J_{v} \pi_{v}\left(u_{i, j}\right) J_{v}$ for all $i, j$.

The net $\left(v_{i, j}\right)_{i, j}$ in $M_{*}^{+}$is increasing because $\left(R_{i, j}^{*} R_{i, j}\right)_{i, j}$ is increasing by assumption on $\left(u_{i}\right)_{i}, v_{i, j}\left(\pi_{v}\left(a^{*} a\right)\right)=\left\|R_{i, j} \Lambda_{v}(a)\right\|^{2}$ for all $a \in A$ and $\pi_{v}(A) \subseteq M$ is weakly dense.

Call $\xi \in H$ right-contractive if $\xi$ is right-bounded and $\left\|R_{\xi}\right\| \leq 1$. Let $x \in M$. Then

$$
\tilde{v}\left(x^{*} x\right)=\sup \left\{\|x \xi\|^{2} \mid \xi \in H \text { is right-contractive }\right\} .
$$

Each $\xi_{i, j}$ is right-contractive by Lemma 3.7.6 and hence $v_{i, j}\left(x^{*} x\right)=\left\|x \Lambda_{v}\left(u_{i, j}\right)\right\|^{2} \leq$ $\tilde{v}\left(x^{*} x\right)$ for all $i, j$. Conversely, for each right-contractive $\xi \in H$,

$$
\begin{aligned}
\|x \xi\|^{2}=\lim _{i, j}\left\|x \pi_{v}\left(u_{i, j}\right) \xi\right\|^{2} & =\lim _{i, j}\left\|x R_{\xi} \Lambda_{v}\left(u_{i, j}\right)\right\|^{2} \\
& \leq \lim _{i, j}\left\|x \Lambda_{v}\left(u_{i, j}\right)\right\|^{2}=\lim _{i, j} v_{i, j}\left(x^{*} x\right)
\end{aligned}
$$

because $R_{\xi} \in M^{\prime}$ and $R_{\xi}^{*} R_{\xi} \leq 1$. Therefore, $\tilde{v}\left(x^{*} x\right) \leq \lim _{i, j} v_{i, j}\left(x^{*} x\right)$.

ii) We only prove the assertion concerning $\tilde{\phi}$. A similar argument as above and Lemma 3.7.6 ii) show that for each $v \in N_{*}^{+}$, the net $\left(v \circ \phi_{i, j}\right)_{i, j}$ is increasing. Taking pointwise limits, we obtain a normal semi-finite weight $\omega$ from $M$ to $N$ such that for each $y \in M$, the element $\omega\left(y^{*} y\right)$ in the extended positive part $\hat{N}_{+}$is defined by $v(\omega(y))=\sup _{i, j} v\left(\phi_{i, j}\left(y^{*} y\right)\right)$ for all $v \in N_{*}^{+}$. Then for all $y \in M$,

$$
\begin{aligned}
\tilde{\mu}\left(\omega\left(y^{*} y\right)\right) \underset{i, j, k}{\nwarrow}\left\|y \Lambda_{\phi}\left(u_{i, j}\right) \Lambda_{\mu}\left(u_{k}\right)\right\|^{2} & =\left\|y \widehat{\beta}\left(\pi_{\mu}\left(u_{k}\right)\right) \xi_{i, j}\right\|^{2} \stackrel{k \rightarrow \infty}{\longrightarrow}\left\|y \xi_{i, j}\right\|^{2} \\
& =v_{i, j}\left(y^{*} y\right) \underset{i, j}{\tilde{v}} \tilde{v}\left(y^{*} y\right)
\end{aligned}
$$

and hence $\tilde{\mu} \circ \omega=\tilde{v}$. By [20, Theorem 4.18], $\omega=\tilde{\phi}$.

Lemma 3.7.8. $W^{*} \rho_{\Lambda_{v}\left(r(b) s\left(b^{\prime}\right)\right)}^{\alpha, \widehat{\beta}} \beta\left(\pi_{\mu}\left(b^{\prime \prime}\right)\right)=\rho_{\Lambda_{v}\left(r\left(b^{\prime \prime}\right) s\left(b^{\prime}\right)\right)}^{\beta, \alpha} \alpha\left(\pi_{\mu}(b)\right)$ for all $b, b^{\prime}, b^{\prime \prime} \in B$.

Proof. Applying both sides to $\Lambda_{v}(a)$, where $a \in A$ is arbitrary, we obtain $W^{*}\left(\Lambda_{v}\left(s\left(b^{\prime \prime}\right) a\right) \underset{\widetilde{\mu}}{\otimes} \Lambda_{v}\left(r(b) s\left(b^{\prime}\right)\right)\right)$ and $\Lambda_{v}(r(b) a) \underset{\widetilde{\mu}}{\otimes} \Lambda_{v}\left(r\left(b^{\prime \prime}\right) s\left(b^{\prime}\right)\right)$, respectively, which coincide.

As usual, let $\mathfrak{N}_{T_{L}}:=\left\{x \in M: T_{L}\left(x^{*} x\right) \in N\right\}$ and similarly define $\mathfrak{N}_{T_{R}}$.

Theorem 3.7.9. Let $\mathcal{A}=(A, \Delta, \epsilon, S, \mu, \phi, \psi)$ be a measured multiplier $(B, \Gamma)$ Hopf $*$-algebroid satisfying the following conditions:

(A1) $\mu$ admits a GNS-representation via bounded operators $\left(K, \Lambda_{\mu}, \pi_{\mu}\right)$, 
(A2) the Radon-Nikodym cocycle of $\mu$ has a positive square root in $M(B)$,

(A3) the bimodule ${ }_{r} A_{s}$ is proper,

(A4) there exists a truncating net for $\mu$.

Let $\tilde{\mu}$ be the weight on $N=\pi_{\mu}(B)^{\prime \prime}$ associated to the Hilbert algebra $\Lambda_{\mu}(B)$, let $\Lambda_{\nu}: A \rightarrow \mathcal{L}(H)$ be the GNS-map for $v=\mu \circ \phi=\mu \circ \psi$, let $\left(\pi_{v}(A)^{\prime \prime}, \alpha, \beta, \Delta\right)$ be the Hopf-von Neumann bimodule of $\mathcal{A}$ (Definition 3.5.7), let $\tilde{v}$ be the weight on $M=\pi_{v}(A)^{\prime \prime}$ associated to the Hilbert algebra $\Lambda_{v}(A)$, and let $T_{L}$ and $T_{R}$ be the n.s.f. weights from $M$ to $\alpha(N)$ and $\beta(N)$ given by $\tilde{\mu} \circ \alpha^{-1} \circ T_{L}=\tilde{v}=\tilde{\mu} \circ \beta^{-1} \circ T_{R}$ (see Proposition 3.7.4). Then $\left(N, \tilde{\mu}, M, \alpha, \beta, \Delta, T_{L}, T_{R}, \tilde{v}\right)$ is a measured quantum groupoid in the sense of [2]. In particular, $T_{L}$ and $T_{R}$ are left-and right-invariant with respect to $\Delta$ in the sense that

$$
\begin{aligned}
& T_{L}\left(\left(\lambda_{\xi}^{\beta, \alpha}\right)^{*} \Delta\left(x^{*} x\right) \lambda_{\xi}^{\beta, \alpha}\right)=\alpha\left(\left(R_{\xi}^{\beta, \tilde{\mu}}\right)^{*} T_{L}\left(x^{*} x\right) R_{\xi}^{\beta, \tilde{\mu}}\right) \\
& \quad \text { for all } x \in \mathfrak{N}_{T_{L}, \xi \in D\left(H_{\beta}, \tilde{\mu}\right),} \\
& T_{R}\left(\left(\rho_{\eta}^{\beta, \alpha}\right)^{*} \Delta\left(x^{*} x\right) \rho_{\eta}^{\beta, \alpha}\right)=\beta\left(\left(R_{\eta}^{\alpha, \tilde{\mu}}\right)^{*} T_{R}\left(x^{*} x\right) R_{\eta}^{\alpha, \tilde{\mu}}\right) \\
& \quad \text { for all } x \in \mathfrak{N}_{T_{R}}, \eta \in D\left(H_{\alpha}, \tilde{\mu}\right) .
\end{aligned}
$$

Proof. We use the same notation as before. To prove the assertion concerning $\tilde{\phi}$ and $T_{L}$, we show that

$$
\left\langle\zeta \mid \tilde{\phi}\left(\left(\lambda_{\xi}^{\beta, \alpha}\right)^{*} \Delta\left(x^{*} x\right) \lambda_{\xi}^{\beta, \alpha}\right) \zeta\right\rangle=\left\|\alpha\left(\tilde{\phi}\left(x^{*} x\right)\right)^{\frac{1}{2}} R_{\xi}^{\beta, \tilde{\mu}} \zeta\right\|^{2}
$$

for all $x \in \mathfrak{N}_{T_{L}}, \xi \in D\left(H_{\beta}, \tilde{\mu}\right)$ and $\zeta \in K$. Given such $x, \xi$, $\zeta$, let

and

$$
\begin{aligned}
\xi_{k} & :=\alpha\left(\pi_{\mu}\left(u_{k}\right)\right) \xi \\
c_{i, j, k} & :=\left\langle\zeta \mid \phi_{i, j}\left(\left(\lambda_{\xi_{k}}^{\beta, \alpha}\right)^{*} \Delta\left(x^{*} x\right) \lambda_{\xi_{k}}^{\beta, \alpha}\right) \zeta\right\rangle \text { for all } i, j, k .
\end{aligned}
$$

Then $R_{\xi_{k}}^{\beta, \tilde{\mu}}=\alpha\left(\pi_{\mu}\left(u_{k}\right)\right) R_{\xi}^{\beta, \tilde{\mu}}, \lambda_{\xi_{k}}^{\beta, \alpha}=\left(\alpha\left(\pi_{\mu}\left(u_{k}\right)\right) \underset{\tilde{\mu}}{\otimes} \mathrm{id}\right) \lambda_{\xi}^{\beta, \alpha}$, and by Proposition 3.7.7,

$$
c_{i, j, k} \stackrel{k \rightarrow \infty}{\longrightarrow}\left\langle\zeta \mid \phi_{i, j}\left(\left(\lambda_{\xi}^{\beta, \alpha}\right)^{*} \Delta\left(x^{*} x\right) \lambda_{\xi}^{\beta, \alpha}\right) \zeta\right\rangle \underset{i, j}{\nearrow}\left\langle\zeta \mid \tilde{\phi}\left(\left(\lambda_{\xi}^{\beta, \alpha}\right)^{*} \Delta\left(x^{*} x\right) \lambda_{\xi}^{\beta, \alpha}\right) \zeta\right\rangle .
$$

On the other hand, using the relation $\Lambda_{\phi}\left(u_{i, j}\right)=\Lambda_{\phi}^{\dagger}\left(u_{i, j}\right)$, we find

$$
\begin{aligned}
& \left.c_{i, j, k}=\left\|(1 \underset{\mu}{\otimes} x) W \lambda_{\xi_{k}}^{\beta, \alpha} \Lambda_{\phi}\left(u_{i, j}\right) \zeta\right\|^{2} \quad \text { (Def. of } \Delta_{W} \text { and } \phi_{i, j}\right) \\
& \left.=\left\|\left(1 \otimes_{\mu}^{\otimes} x\right) W \rho_{\Lambda_{v}\left(u_{i, j}\right)}^{\beta, \alpha} \alpha\left(\pi_{\mu}\left(u_{k}\right)\right) R_{\xi}^{\beta, \tilde{\mu}} \zeta\right\|^{2} \quad \text { (Def. of } H_{\beta} \underset{\tilde{\mu}}{\otimes_{\alpha}} H\right) \\
& =\left\|(1 \underset{\mu}{\otimes} x) \rho_{\Lambda_{v}\left(u_{k, j}\right)}^{\widehat{\alpha}, \beta} \beta\left(\pi_{\mu}\left(u_{i}\right)\right) R_{\eta}^{\beta, \tilde{\mu}} \zeta\right\|^{2} \quad \text { (Lem. 3.7.8) } \\
& =\left\|\widehat{\alpha}\left(\phi_{k, j}\left(x^{*} x\right)\right)^{\frac{1}{2}} \beta\left(\pi_{\mu}\left(u_{i}\right)\right) R_{\xi}^{\beta, \tilde{\mu}} \zeta\right\|^{2} \underset{i, j, k}{\nearrow}\left\|\widehat{\alpha}\left(\tilde{\phi}\left(x^{*} x\right)\right)^{\frac{1}{2}} R_{\xi}^{\beta, \tilde{\mu}} \zeta\right\|^{2} .
\end{aligned}
$$


Thus, (3.23) follows. The assertion concerning $\tilde{\psi}$ and $T_{R}$ can be proven similarly, where $W$ has to be replaced by the unitary $V$.

Remark 3.7.10. Assume that $\phi=(\mathrm{id} \otimes \mu) \circ h$ for a normalized bi-integral $h$ on $(A, \Delta)$. Then for each $b \in B$, the map $\Lambda_{\mu}(B) \rightarrow \Lambda_{v}(A)$ given by $\Lambda_{\mu}(c) \mapsto \Lambda_{v}(s(b) r(c))$ is bounded with norm less than or equal to $\mu\left(b^{*} b\right)^{\frac{1}{2}}$, and therefore extends to an operator $\Lambda_{\phi}(s(b)) \in \mathcal{L}(K, H)$. One can then approximate $\tilde{\phi}$ monotonously by the maps $\phi_{i}: M \rightarrow N, x \mapsto \Lambda_{\phi}\left(s\left(u_{i}\right)\right)^{*} x \Lambda_{\phi}\left(s\left(u_{i}\right)\right)$, and a similar calculation as in Lemma 3.7.8 shows that each $\phi_{i}$ is right-invariant.

Associated to the measured quantum groupoid $\left(N, \tilde{\mu}, M, \alpha, \beta, \Delta, T_{L}, T_{R}, \tilde{v}\right)$ are two fundamental unitaries $U_{H}^{\prime}: H_{\widehat{\alpha}} \otimes_{\tilde{\mu}} \beta H \rightarrow H_{\beta} \underset{\tilde{\mu}}{\otimes_{\alpha}} H$ and $U_{H}: H_{\alpha} \underset{\tilde{\mu}}{\otimes_{\widehat{\beta}}} H \rightarrow$ $H_{\beta} \underset{\tilde{\mu}}{\underset{\alpha}{\alpha}} H$, characterized by

$$
\begin{aligned}
&\left(\lambda_{w}^{\beta, \alpha}\right)^{*} U_{H}\left(v \underset{\tilde{\mu}}{\otimes} \Lambda_{\tilde{v}}(a)\right)= \Lambda_{\tilde{\nu}}\left(\left(\omega_{w, v} * \operatorname{id}\right)(\Delta(a))\right) \\
& \quad \text { for all } v, w \in D\left(H_{\beta}, \tilde{\mu}\right), a \in \mathfrak{N}_{\tilde{v}} \cap \mathfrak{N}_{T_{L}}, \\
&\left(\rho_{w^{\prime}}^{\beta, \alpha}\right)^{*} U_{H}^{\prime}\left(\Lambda_{\tilde{\nu}}\left(a^{\prime}\right) \underset{\tilde{\mu}}{\otimes} v^{\prime}\right)=\Lambda_{\tilde{v}}\left(\left(\operatorname{id} * \omega_{w^{\prime}, v^{\prime}}\right)\left(\Delta\left(a^{\prime}\right)\right)\right), \\
& \quad \text { for all } v^{\prime}, w^{\prime} \in D\left(H_{\alpha}, \tilde{\mu}\right), a^{\prime} \in \mathfrak{N}_{\tilde{v}} \cap \mathfrak{N}_{T_{R}} ;
\end{aligned}
$$

see [13, Proposition 3.17].

Proposition 3.7.11. $W^{*}=U_{H}$ and $V=U_{H}^{\prime}$.

Proof. Let $x, y, y^{\prime}, z \in A$ and choose $v_{i}, w_{i} \in A$ such that $\sum \bar{D}^{\frac{1}{2}}\left(y_{(1)}\right) x^{\prime} \underset{B}{\otimes} y_{(2)}=$ $\sum v_{i} \underset{B}{\otimes} w_{i}$ in ${ }_{s} A \underset{B}{\otimes} r$. Then

$$
\begin{aligned}
\left(\omega_{\Lambda_{v}(x), \Lambda_{v}\left(x^{\prime}\right)} * \operatorname{id}\right)\left(W^{*}\right) \Lambda_{v}(y) & =\sum_{i}\left(\lambda_{\Lambda_{v}(x)}^{\beta, \alpha}\right)^{*}\left(\Lambda_{v}\left(v_{i}\right) \underset{\tilde{\mu}}{\otimes} \Lambda_{v}\left(w_{i}\right)\right) \\
& =\sum_{i} \Lambda_{v}\left(r\left(\psi\left(v_{i} \theta\left(x^{*}\right)\right)\right) w_{i}\right) \\
\left(\omega_{\Lambda_{v}(x), \Lambda_{v}\left(x^{\prime}\right)} * \operatorname{id}\right)(\Delta(y)) \Lambda_{v}(z) & =\sum_{i}\left(\lambda_{\Lambda_{v}(x)}^{\beta, \alpha}\right)^{*}\left(\Lambda_{v}\left(v_{i}\right) \underset{\widetilde{\mu}}{\otimes} \Lambda_{v}\left(w_{i} z\right)\right) \\
& =\sum_{i} \pi_{v}\left(r\left(\psi\left(v_{i} \theta\left(x^{*}\right)\right)\right)\right) \Lambda_{v}\left(w_{i} z\right),
\end{aligned}
$$

and hence $\left(\omega_{\Lambda_{v}(x), \Lambda_{v}\left(x^{\prime}\right)} * \operatorname{id}\right)\left(W^{*}\right) \Lambda_{v}(y)=\Lambda_{\tilde{v}}\left(\left(\omega_{\Lambda_{v}(x), \Lambda_{v}}\left(x^{\prime}\right) * \operatorname{id}\right)(\Delta(y))\right)$. 
Likewise, with $v_{i}^{\prime}, w_{i}^{\prime} \in A$ such that $\sum \bar{D}^{\frac{1}{2}}\left(x_{(1)}\right) \underset{B}{\otimes} x_{(2)} y^{\prime}=\sum v_{i}^{\prime} \underset{B}{\otimes} w_{i}^{\prime} \in{ }_{s} A \underset{B}{\otimes} r A$, we find

$$
\begin{aligned}
\left(\operatorname{id} * \omega_{\Lambda_{v}(y), \Lambda_{v}\left(y^{\prime}\right)}\right)(V) \Lambda_{v}(x) & =\sum_{i}\left(\rho_{\Lambda_{v}(y)}^{\beta, \alpha}\right)^{*}\left(\Lambda_{v}\left(v_{i}^{\prime}\right) \underset{\widetilde{\mu}}{\otimes} \Lambda_{v}\left(w_{i}^{\prime}\right)\right) \\
& =\sum_{i} \Lambda_{v}\left(s\left(\phi\left(w_{i}^{\prime} \theta\left(y^{*}\right)\right)\right) v_{i}^{\prime}\right), \\
\left(\operatorname{id} * \omega_{\Lambda_{v}\left(y^{\prime}\right), \Lambda_{v}(y)}\right)\left(\Delta\left(\pi_{v}(x)\right)\right) \Lambda_{v}(z) & =\sum_{i}\left(\rho_{\Lambda_{v}(y)}^{\beta, \alpha}\right)^{*}\left(\Lambda_{v}\left(v_{i}^{\prime} z\right) \underset{\widetilde{\mu}}{\otimes} \Lambda_{v}\left(w_{i}^{\prime}\right)\right) \\
& =\sum_{i} \pi_{v}\left(s\left(w_{i}^{\prime} \theta\left(y^{*}\right)\right)\right) \Lambda_{v}\left(v_{i}^{\prime} z\right)
\end{aligned}
$$

and hence $\left(\operatorname{id} * \omega_{\Lambda_{v}(y), \Lambda_{v}\left(y^{\prime}\right)}\right)(V) \Lambda_{v}(x)=\Lambda_{\tilde{v}}\left(\left(\operatorname{id} * \omega_{\Lambda_{v}\left(y^{\prime}\right), \Lambda_{v}(y)}\right)\left(\Delta\left(\pi_{v}(x)\right)\right)\right)$.

The adapted measured quantum groupoid $\left(N, \tilde{\mu}, M, \alpha, \beta, \Delta, T_{L}, T_{R}, \tilde{v}\right)$ has an antipode $\tilde{S}$ which is characterized by the following properties:

i) $\operatorname{span}\left\{\left(\omega_{v, w} * \mathrm{id}\right)(V): w, v \in \mathcal{T}_{\tilde{v}, T_{R}}\right\}$ is a core for $\tilde{S}$,

ii) $\tilde{S}\left(\left(\omega_{w, v} * \mathrm{id}\right)(V)\right)=\left(\omega_{w, v} * \mathrm{id}\right)\left(V^{*}\right)$ for all $w, v \in \mathcal{T}_{\tilde{v}, T_{R}}$,

where $\mathcal{T}_{\tilde{v}, T_{R}}$ is the set of all $x \in M$ that are analytic with respect to $\sigma^{\tilde{\nu}}$ and satisfy $\sigma_{z}^{\tilde{v}} \in \mathfrak{N}_{\tilde{v}} \cap \mathfrak{N}_{\tilde{v}}^{*} \cap \mathfrak{N}_{T_{R}} \cap \mathfrak{N}_{T_{R}}^{*}$ for all $z \in \mathbb{C}$. Likewise, one defines $\mathcal{T}_{\tilde{v}, T_{L}}$.

Lemma 3.7.12. $\pi_{v}(A) \subseteq \mathcal{T}_{\tilde{v}, T_{R}} \cap \mathcal{T}_{\tilde{v}, T_{L}}$.

Proof. Recall that $\pi_{v}(A) \subseteq \mathcal{T}_{\tilde{v}}$ by Proposition 3.7.2. Using Lemma 3.7.3 i), we find

$$
\sigma_{z}^{\tilde{\nu}}\left(\pi_{v}(A)\right)=\sigma_{z}^{\tilde{\nu}}\left(\pi_{v}(A s(B))\right)=\sigma_{z}^{\tilde{\nu}}\left(\pi_{v}(A)\right) \beta\left(\pi_{\mu}(B)\right) \subseteq \mathfrak{N}_{\tilde{\nu}} \beta\left(\mathfrak{N}_{\tilde{\mu}}\right) \subseteq \mathfrak{N}_{T_{R}}
$$

for all $z \in \mathbb{C}$. Consequently, $\pi_{v}(A) \subseteq \mathcal{T}_{\tilde{v}, T_{R}}$. A similar argument shows that $\pi_{v}(A) \subseteq \mathcal{T}_{\tilde{v}, T_{L}}$.

Proposition 3.7.13. $\pi_{v}(A) \subseteq \operatorname{Dom}(\tilde{S})$ and $\tilde{S}\left(\pi_{v}(a)\right)=\pi_{v}\left(D^{\frac{1}{2}} S D^{\frac{1}{2}}(a)\right)$ for all $a \in A$. 
Proof. Let $x, x^{\prime} \in A$ and $a=\sum D^{-\frac{1}{2}}\left(x_{(2)}^{\prime} r\left(\psi\left(x^{*} x_{(1)}^{\prime}\right)\right)\right)$. Then

$$
\begin{aligned}
& \left(\omega_{\Lambda_{v}(x), \Lambda_{v}\left(x^{\prime}\right)} * \mathrm{id}\right)(V)=\pi_{v}(a), \\
& \left(\omega_{\Lambda_{v}(x), \Lambda_{v}\left(x^{\prime}\right)} * \mathrm{id}\right)\left(V^{*}\right)=\left(\left(\lambda_{\Lambda_{v}\left(x^{\prime}\right)}^{\beta, \alpha}\right) * \lambda_{\Lambda_{v}(x)}^{\widehat{\alpha}, \beta}\right)^{*} \\
& =\sum \pi_{v}\left(D^{-\frac{1}{2}}\left(x_{(2)} r\left(\psi\left(x^{\prime *} x_{(1)}\right)\right)\right)\right)^{*} \\
& =\sum \pi_{v}\left(D^{\frac{1}{2}}\left(r\left(\psi\left(x_{(1)}^{*} x^{\prime}\right)\right) x_{(2)}^{*}\right)\right) \\
& =\sum \pi_{\nu}\left(D^{\frac{1}{2}}\left(S\left(x_{(2)}^{\prime} r\left(\psi\left(x^{*} x_{(1)}^{\prime}\right)\right)\right)\right)\right) \quad \text { (Prop. 2.5.3) } \\
& =\pi_{v}\left(D^{\frac{1}{2}} S D^{\frac{1}{2}}(a)\right) \text {. }
\end{aligned}
$$

Acknowledgements. I thank Erik Koelink for introducing me to dynamical quantum groups and for stimulating discussion, and the referee for very helpful remarks that improved the presentation.

\section{References}

[1] G. Böhm. Hopf algebroids. In Handbook of algebra. Vol. 6, volume 6 of Handb. Algebr., pages 173-235. Elsevier/North-Holland, Amsterdam, 2009. Zbl 1220.16022 MR 2553659

[2] M. Enock. Measured quantum groupoids in action. Mém. Soc. Math. Fr. (N.S.), (114):ii+150 pp. (2009), 2008. Zbl 1189.58002 MR 2541012

[3] M. Enock. Inclusions of von Neumann algebras and quantum groupoids III. J. Funct. Anal., 223(2):311-364, 2005. Zbl 1088.46036 MR 2142344

[4] M. Enock and R. Nest. Irreducible inclusions of factors, multiplicative unitaries, and Kac algebras. J. Funct. Anal., 137(2):466-543, 1996. Zbl 0847.22003 MR 1387518

[5] P. Etingof and D. Calaque. Lectures on tensor categories. In Quantum groups, volume 12 of IRMA Lect. Math. Theor. Phys., pages 1-38. Eur. Math. Soc., Zürich, 2008. Zbl 1160.18004 MR 2432988

[6] P. Etingof and F. Latour. The dynamical Yang-Baxter equation, representation theory, and quantum integrable systems, volume 29 of Oxford Lecture Series in Mathematics and its Applications. Oxford University Press, Oxford, 2005. Zbl 1071.17012 MR 2142557

[7] P. Etingof and A. Varchenko. Solutions of the quantum dynamical Yang-Baxter equation and dynamical quantum groups. Comm. Math. Phys., 196(3):591-640, 1998. Zbl 0957.17019 MR 1645196

[8] P. Etingof and A. Varchenko. Exchange dynamical quantum groups. Comm. Math. Phys., 205(1):19-52, 1999. Zbl 0943.17010 MR 1706916

[9] L. Kadison and K. Szlachányi. Bialgebroid actions on depth two extensions and duality. Adv. Math., 179(1):75-121, 2003. Zbl 1049.16022 MR 2004729 
[10] E. Koelink and H. Rosengren. Harmonic analysis on the SU(2) dynamical quantum group. Acta Appl. Math., 69(2):163-220, 2001. Zbl 1011.43003 MR 1872106

[11] J. Kustermans and A. van Daele. $C^{*}$-algebraic quantum groups arising from algebraic quantum groups. Internat. J. Math., 8(8):1067-1139, 1997. Zbl 1009.46038 MR 1484867

[12] J. Kustermans and A. van Daele. $C^{*}$-algebraic quantum groups arising from algebraic quantum groups. Internat. J. Math., 8(8):1067-1139, 1997. Zbl 1009.46038 MR 1484867

[13] F. Lesieur. Measured quantum groupoids. Mém. Soc. Math. Fr. (N.S.), (109):iv+158 pp. (2008), 2007. Zbl 1221.46003 MR 2474165

[14] D. Nikshych and L. Vainerman. Algebraic versions of a finite-dimensional quantum groupoid. In Hopf algebras and quantum groups (Brussels, 1998), volume 209 of Lecture Notes in Pure and Appl. Math., pages 189-220. Dekker, New York, 2000. Zbl 1032.46537 MR 1763613

[15] D. Nikshych and L. Vainerman. A characterization of depth 2 subfactors of $\mathrm{II}_{1}$ factors. $J$. Funct. Anal., 171(2):278-307, 2000. Zbl 1010.46063 MR 1745634

[16] J.-L. Sauvageot. Produits tensoriels de $Z$-modules et applications. In Operator algebras and their connections with topology and ergodic theory (Bușteni, 1983), volume 1132 of Lecture Notes in Mathematics, pages 468-485. Springer, Berlin, 1985. Zbl 0577.46062 MR 799587

[17] P. Schauenburg. Weak Hopf algebras and quantum groupoids. In Noncommutative geometry and quantum groups (Warsaw, 2001), volume 61 of Banach Center Publ., pages 171-188. Polish Acad. Sci., Warsaw, 2003. Zbl 1064.16041 MR 2024429

[18] Ş. Strătilă. Modular theory in operator algebras. Editura Academiei Republicii Socialiste România, Bucharest, 1981. Translated from the Romanian by the author. Zbl 0504.46043 MR 696172

[19] K. Szlachányi. Finite quantum groupoids and inclusions of finite type. In Mathematical physics in mathematics and physics (Siena, 2000), volume 30 of Fields Inst. Commun., pages 393-407. Amer. Math. Soc., Providence, RI, 2001. Zbl 1022.18007 MR 1867570

[20] M. Takesaki. Theory of operator algebras. II, volume 125 of Encyclopaedia of Mathematical Sciences. Springer-Verlag, Berlin, 2003. Zbl 1059.46031 MR 1943006

[21] T. Timmermann. Free dynamical quantum groups and the dynamical quantum group $\mathrm{SU}_{q}^{\mathrm{d}}(2)$ in Operator algebras and quantum groups, 311-341, Banach Center Publ., 98, Polish Acad. Sci., Inst. Math., Warsaw, 2012. Zbl 1272.81099 MR 3059668

[22] T. Timmermann. The relative tensor product and a minimal fiber product in the setting of $C^{*}$-algebras. J. Operator Theory, 68(2):365-404, 2012. Zbl 1289.46087 MR 2995727

[23] T. Timmermann. An invitation to quantum groups and duality. EMS Textbooks in Mathematics. European Mathematical Society (EMS), Zürich, 2008. Zbl 1162.46001 MR 2397671

[24] T. Timmermann. $C^{*}$-pseudo-multiplicative unitaries, Hopf $C^{*}$-bimodules and their Fourier algebras. J. Inst. Math. Jussieu, 11:189-229, 2011. Zbl 1238.46053 MR 2862379

[25] T. Timmermann and A. Van Daele. Regular multiplier Hopf algebroids. Basic theory and examples. In preparation, arXiv:1307.0769, 2014. 
[26] J.-M. Vallin. Bimodules de Hopf et poids opératoriels de Haar. J. Operator Theory, 35(1):39-65, 1996. Zbl 0849.22002 MR 1389642

[27] J.-M. Vallin. Unitaire pseudo-multiplicatif associé à un groupoïde. Applications à la moyennabilité. J. Operator Theory, 44(2):347-368, 2000. Zbl 0986.22002 MR 1794823

[28] J.-M. Vallin. Groupoïdes quantiques finis. J. Algebra, 239(1):215-261, 2001. Zbl 1003.46040 MR 1827882

[29] J.-M. Vallin. Measured quantum groupoids associated with matched pairs of locally compact groupoids, 2009.

[30] A. van Daele. Multiplier Hopf algebras. Trans. Amer. Math. Soc., 342(2):917-932, 1994. Zbl 0809.16047 MR 1220906

[31] A. van Daele. An algebraic framework for group duality. Adv. Math., 140(2):323-366, 1998. Zbl 0933.16043 MR 1658585

[32] A. Van Daele. Tools for working with multiplier Hopf algebras. Arab. J. Sci. Eng. Sect. C Theme Issues, 33(2):505-527, 2008. Zbl 1186.16027 MR 2500055

[33] A. Van Daele and S. Wang. Weak multiplier Hopf algebras. Preliminaries, motivation and basic examples. In Operator Algebras and Quantum Groups (Warsaw, 2011), volume 98 of Banach Center Publ., pages 367-415. Polish Acad. Sci., Warsaw, 2012. Zbl 1267.16030 MR 3059670

[34] A. Van Daele and S. Wang. Weak multiplier Hopf algebras. The main theory. arXiv:1210.4395, 2012.

[35] S. L. Woronowicz. Compact quantum groups. In Symétries quantiques (Les Houches, 1995), pages 845-884. North-Holland, Amsterdam, 1998. Zbl 0997.46045 MR 1616348

Received 27 September, 2012; revised 20 April, 2013

T. Timmermann, University of Muenster, Einsteinstr. 62, 48149 Muenster, Germany

E-mail: timmermt@uni-muenster.de 